\title{
Hedonic Games with Ordinal Preferences and Thresholds
}

\author{
Anna Maria Kerkmann \\ Heinrich-Heine-Universität Düsseldorf \\ 40225 Düsseldorf, Germany \\ Jérôme Lang \\ LAMSADE, CNRS, Université Paris-Dauphine, PSL \\ 75775 Paris Cedex 16, France
}

\author{
Anja Rey \\ Technische Universität Dortmund \\ 44221 Dortmund, Germany \\ Jörg Rothe \\ Hilmar Schadrack \\ Lena Schend \\ Heinrich-Heine-Universität Düsseldorf \\ 40225 Düsseldorf, Germany
}

\author{
ANNA.KERKMANN@HHU.DE
}

LANG@LAMSADE.DAUPHINE.FR

ANJA.REY@TU-DORTMUND.DE

ROTHE@HHU.DE

HILMAR.SCHADRACK@HHU.DE

LENA.SCHEND@GOOGLEMAIL.COM

\begin{abstract}
We propose a new representation setting for hedonic games, where each agent partitions the set of other agents into friends, enemies, and neutral agents, with friends and enemies being ranked. Under the assumption that preferences are monotonic (respectively, antimonotonic) with respect to the addition of friends (respectively, enemies), we propose a bipolar extension of the responsive extension principle, and use this principle to derive the (partial) preferences of agents over coalitions. Then, for a number of solution concepts, we characterize partitions that necessarily or possibly satisfy them, and we study the related problems in terms of their complexity.
\end{abstract}

\section{Introduction}

Hedonic games are cooperative games where agents form coalitions. Each agent has a preference relation over the set of all coalitions containing her. Various solution concepts—such as individual rationality, Nash stability, individual stability, core stability, popularity, and so on-have been proposed and studied for hedonic games. These solution concepts apply to coalition structures, that is, to partitions of the set of agents into disjoint coalitions. For instance, a coalition structure is individually rational if no agent prefers the coalition of which she is the only member to the coalition she is currently a member of, and it is Nash stable if no agent prefers to be integrated into another existing coalition than staying in her current coalition. (Other solution concepts will be explained later on.)

\subsection{Hedonic Games: Standard Game-Theoretic vs. Engineering-Oriented Point of View}

There are two different points of view under which we can study hedonic games. Under a standard game-theoretic point of view, a hedonic game is a model by which one can predict (or at least reason about) the coalitions that a set of agents, acting without the intervention of a central authority, may form given what we know their preferences. Under an engineering-oriented point of view, a game 
is the input of a problem whose output, computed by a central authority, is a set of coalitions, which should be as satisfactory as possible.

Both the origin and the description of the game differ under these two interpretations of hedonic games. Under the standard game-theoretic one, the game is written down by the modeler; under the engineering-oriented one, it is written down by the agents, each of which has to report their preferences on the possible coalitions they may end up forming.

The role of solution concepts is also different under these two interpretations. Under the standard game-theoretic one, solution concepts are assumed to be realistic models of what may happen; for instance, if a partition $\Gamma$ of the agents is not individually rational (that is, at least one agent would be better leaving her coalition and be alone), and if agents are free to move out and form singleton coalitions, then it is not reasonable to expect that $\Gamma$ will be the final outcome of the game; as another example, if agents can choose to leave their coalition and join any other existing coalition without asking for permission of the coalition they join, nor of the coalition they leave, then Nash stability is a relevant solution concept, and therefore, provided there exists at least a Nash stable coalition structure, we can expect that the resulting coalition structure will be Nash stable (and if there exists no Nash stable coalition structure, we can predict that the outcome of the game will be unstable).

Under the engineering-oriented interpretation, solution concepts are desiderata that one may impose on the outcome: For instance, individual rationality should be a hard constraint that the outcome must satisfy. In this paper, we focus on this interpretation of hedonic games. Therefore, our assumption will be that a central authority has first to elicit the agents' preferences about coalitions, and then to compute a desirable outcome. In the following paragraph, we will discuss the difficulties inherent to these two stages (elicitation and computation). Our contribution mostly consists in defining a new framework for hedonic games that comes with a representation language that offers a trade-off between expressivity and succinctness, and to study various stability notions in this setting.

\subsection{Representing Preferences over Coalitions}

Since each agent has to specify a preference relation over the set of all coalitions containing her, an important bottleneck is how the agents' preferences over the coalitions that contain them are expressed. As there are exponentially many (in the number of agents) coalitions containing agent $i$, it is not reasonable to expect agent $i$ to express a ranking (or a utility function) over all these coalitions explicitly. This issue is often addressed by assuming that only a small part of the preference relation is expressed by the agent, and that this small part is then extended to a complete preference relation over coalitions using an appropriate extension principle. Various assumptions about the nature of the input (specifying what the agents are required to express) and the preference extension have been made in the literature (for recent surveys, see Aziz \& Savani, 2016; Elkind \& Rothe, 2015; Woeginger, 2013a):

1. The individually rational encoding (Ballester, 2004): Each agent explicitly ranks all coalitions she prefers to herself being alone, and only those ones.

2. Hedonic coalition nets (Elkind \& Wooldridge, 2009): Each agent specifies her utility function over the set of all coalitions via (more or less) a set of weighted logical formulas.

3. The singleton encoding (Cechlárová \& Romero-Medina, 2001; Cechlárová \& Hajduková, 2003, 2004): Each agent ranks only single agents; under the optimistic (respectively, pes- 
simistic) extension, $X$ is preferred to $Y$ if the best (respectively, worst) agent in $X$ is preferred to the best (respectively, worst) agent in $Y$.

4. The additive encoding (Sung \& Dimitrov, 2007, 2010; Aziz et al., 2013b; Woeginger, 2013b): Each agent gives a valuation (positive or negative) of each other agent; preferences are additively separable, and the extension principle is that the valuation of a set of agents, for agent $i$, is the sum of the valuations $i$ gives to the agents in the set (and then the preference relation is derived from this valuation function).

5. Fractional hedonic games (Aziz et al., 2019; Bilò et al., 2014, 2015): Once again, each agent assigns a value to each other agent (and 0 to herself); an agent's utility of a coalition is the average value she assigns to the members of the coalition.

6. The friends-and-enemies encoding (Dimitrov et al., 2006; Sung \& Dimitrov, 2007; Rey et al., 2016; Nguyen et al., 2016): Each agent partitions the set of other agents into two sets (her friends and her enemies); under the friend-oriented preference extension, coalition $X$ is preferred to coalition $Y$ if $X$ contains more friends than $Y$, or as many friends as $Y$ and fewer enemies than $Y$; under the enemy-oriented preference extension, $X$ is preferred to $Y$ if $X$ contains fewer enemies than $Y$, or as many enemies as $Y$ and more friends than $Y$.

7. The anonymous encoding (Ballester, 2004; Darmann et al., 2018): Each agent specifies only a preference relation over the number of agents in her coalition (ignoring who they are).

8. Boolean hedonic games (Aziz et al., 2016; Peters, 2016): Each agent partitions all coalitions into two subsets one of which she prefers to the other while being indifferent between the coalitions inside each of those two subsets. This partition is expressed compactly in propositional logic.

We can classify these various ways of specifying hedonic games according to two parameters:

- the nature of the output: ordinal (for each agent $i$, a preference relation over coalitions containing $i$ ), cardinal (a utility function over coalitions containing $i$ ), or dichotomous (a partition of coalitions containing $i$ between good and bad ones);

- the nature of the language used for expressing the agent's preference over coalitions containing her: explicit (coalitions are listed in extension), logical (preferences are expressed using logical formulas or similar objects), singleton-wise (only single agents are ranked or given a value), or anonymous (preferences are expressed only on possible cardinalities of coalitions).

In Table 1 we classify each of our languages along these two parameters, where the numbers in the table correspond to the above enumeration of eight encodings of hedonic games.

Naturally, compact representation either does not avoid exponential-size representations in the worst case (Case 1 and, to a lesser extent, Case 2), or comes with a loss of expressivity, corresponding to a demanding domain restriction, such as separable preferences (Cases 3, 4, and 5), anonymous preferences (Case 7), or other domain restrictions that do not bear a specific name (Cases 6 and 8).

In Cases 2, 4, and 5, preferences are expressed numerically: Agents do explicitly express numbers. In all other cases, they are expressed ordinally. The difficulties with eliciting and aggregating numeric preferences have been long discussed in social choice (Sen, 1970), and for these reasons the community favors ordinal preferences.

Anonymity is a very demanding assumption, which does not allow to distinguish between agents. Even if it makes sense in some settings, such as in group activity selection (Darmann et al., 2018), it is unrealistic in most cases. The individually rational encoding is not compact in general. 


\begin{tabular}{lccc}
\hline & dichotomous & ordinal & cardinal \\
\hline explicit & & 1 & \\
logical & 8 & & 2 \\
singleton-wise & 6 & 3 & 4,5 \\
anonymous & & 7 & \\
\hline
\end{tabular}

Table 1: Classification of some representation languages for hedonic games. They are referred to by their numbers in the enumeration.

\subsection{Solution Concepts and Computation}

While one difficulty we wish to overcome has to do with the space, time, and cognitive effort required from the agents for expressing their preferences, another difficulty consists in the complexity of computing coalition structures that satisfy some solution concept, or, when such a coalition structure is not guaranteed to exist, to check whether there exists a coalition structure that satisfies it. Because of the exponential number of possible coalition structures, there is no guarantee that these problems are easy to solve, and indeed in many cases they are hard. A lot of attention has been devoted to the computational complexity of the problems associated with various solution concepts under different representations; they are surveyed in Section 15.4 of the book chapter by Aziz and Savani (2016) and in Section 3.3.3 of the book chapter by Elkind and Rothe (2015).

\subsection{Towards a More Satisfactory Representation}

As discussed before, desiderata for hedonic game representations are expressivity, succinctness, and cognitive simplicity. We would like, ideally, a representation that satisfies the following three requirements, or that, at least, is a satisfactory trade-off between them:

(1) it should be reasonably expressive;

(2) it should be compact;

(3) it should be cognitively plausible, and easy to elicit from the agents.

Because of requirement (3), we want to stick to ordinal preferences which, among other advantages, are easier to elicit from the agents. Requirement (1) excludes the very demanding anonymity assumption, which does not allow to distinguish between agents. Requirement (2) excludes the individually rational encoding, which is not compact in general.

The only remaining representations are the friends-and-enemies and singleton encodings. However, we argue that they are insufficiently expressive and thus are poor on requirement (1). A problem with the friends-and-enemies encoding is that an agent cannot express preferences inside the friend set nor inside the enemy set: Preferences over individual agents are dichotomous (but preferences between coalitions are not, because they depend on the number of friends and enemies). A problem with the singleton encoding is that having simply a rank $\triangleright_{i}$ for each agent $i$ does not tell us which agents $i$ would like to see in her coalition and which agents she would like not to see: For instance, if $\triangleright_{1}$ is $2 \triangleright_{1} 3 \triangleright_{1} 4$, we know that 1 prefers 2 to 3 and 3 to 4 , but nothing tells us whether 1 


\begin{tabular}{lcccc}
\hline & dichotomous & ordinal & polarized ordinal & cardinal \\
\hline explicit & & 1 & & \\
logical & 8 & & & 2 \\
singleton-wise & 6 & 3 & this work & 4,5 \\
anonymous & & 7 & & \\
\hline
\end{tabular}

Table 2: Classification of some representation languages for hedonic games, including ours. The representation languages are again referred to by their numbers in the enumeration above.

prefers to be with 2 (respectively, with 3 and 4 ) to being alone, that is, whether the "absolute desirability" of 2, 3, and 4 is positive or negative (of course, if it is negative for 3 , it is also negative for 4 , etc.). Obviously, both ways are insufficiently informative: Specifying only a partition into favorable and disliked agents ("friends" and "enemies") does not tell which of her friends $i$ prefers to which other agents, and which of her enemies she wants to avoid most. On the other hand, specifying a ranking over agents does not say which agents $i$ prefers to be with rather than being alone.

Therefore, we propose a model that integrates the models of Cases 3 and 6 (as described in the list in Section 1.2 on pages 706-707): Each agent $i$ first subdivides the other agents into three groups-her friends, her enemies, and an intermediate type of agent on which she has neither a positive nor a negative opinion-and then specifies a ranking of her friends and a ranking of her enemies. Our new representation of hedonic games with friends, enemies, and neutral players is called the model of FEN-hedonic game.

This representation is not purely ordinal: Along with the preference relation we have a threshold that indicates which coalitions are better than being alone, which ones are worse, and which ones are equally good. Such a structure, which we call polarized ordinal, is reminiscent of "approvalranking" ballots in fallback voting (Brams \& Sanver, 2009), where each voter ranks candidates along with indicating an approval threshold. Table 2 also shows where our representation method is located with respect to the two parameters mentioned earlier.

Based on this representation, we consider a natural extension of a player's preference, the polarized responsive extension, which is a partial order over coalitions containing the player.

Responsive preferences come from bipartite many-to-one matching markets (see, e.g., Roth, 1985; Roth \& Sotomayor, 1992), and consider the comparison of one participant to another. In the context of many-to-one matching markets, an agent on the one side has responsive preferences over assignments of the agents on the other side if, for any two assignments that differ in only one agent, the assignment containing the most preferred agent is preferred. The responsive extension principle is sometimes called the Bossong-Schweigert extension principle (Bossong \& Schweigert, 2006) (see also Delort et al., 2011).

How can we deal with incomparabilities within these partial orders? Our approach is to leave them open and define notions such as "possible" and "necessary" stability concepts. Questions of interest include appropriate characterizations of stability concepts and a computational study of the related problems in terms of their complexity. 


\subsection{Outline}

This paper is a largely extended version of its conference predecessors (Lang, Rey, Rothe, Schadrack, \& Schend, 2015; Kerkmann \& Rothe, 2019). It contains all omitted proofs, as well as a broad spectrum of new insights, results, and discussions. This includes a more detailed discussion of our model and its advantages in comparison to existing models. We will also extend the axiomatic analysis of the different steps of the preference extensions, and use this analysis to further delimit our model to other models of hedonic game. On the other hand, our results on Borda-induced hedonic games from the conference version (Lang et al., 2015) are not contained here but have appeared in a separate journal article (Rothe, Schadrack, \& Schend, 2018).

After introducing the needed notions and definitions in Section 2, explaining various representations of and stability concepts for hedonic games as well as some required terms from complexity theory, we turn in Section 3 to our new model of FEN-hedonic games, using ordinal preferences with double threshold and the polarized responsive principle. In Section 4, we study stability in FEN-hedonic games, in particular pinpointing the complexity of possible and necessary stability problems. In Section 5, we conclude our work and give a brief overview of open problems and possible future work.

\section{Preliminaries}

A hedonic game is a pair $(A, \succeq)$ consisting of a set of players (or agents) $A=\{1,2, \ldots, n\}$ and a profile of preference relations $\succeq=\left(\succeq_{1}, \succeq_{2}, \ldots, \succeq_{n}\right)$ defining for each player a weak preference order over all possible coalitions $C \subseteq A$ containing the player herself. ${ }^{1}$ We denote the set of all coalitions containing player $i \in A$ with $\mathscr{A}_{i}$. For two coalitions $C, D \in \mathscr{A}_{i}$, we say that $i$ weakly prefers $C$ to $D$ if $C \succeq_{i} D$; i prefers $C$ to $D$, denoted by $C \succ_{i} D$, if $C \succeq_{i} D$ but not $D \succeq_{i} C$; and $i$ is indifferent between $C$ and $D$, denoted by $C \sim_{i} D$, if both $C \succeq_{i} D$ and $D \succeq_{i} C$. A coalition structure $\Gamma$ for a given game $(A, \succeq)$ is a partition of $A$ into disjoint coalitions, and for each player $i \in A, \Gamma(i)$ denotes the unique coalition in $\Gamma$ containing $i$. We denote the set of all possible coalition structures for a hedonic game $(A, \succeq)$ by $\mathscr{C}_{(A, \succeq)}$. Occasionally, we may omit the hedonic game in this notion and just write $\mathscr{C}$ if this is clear from the context.

\subsection{Some Known Representations of Hedonic Games}

The need for a succinct representation of hedonic games calls for the definition of a compact representation language for preferences over coalitions containing a player. Specifically, using this language players should be required to express their preferences in a compact manner. At the same time, they should have the opportunity to express them in as much detail as possible. To address this issue, a number of sophisticated approaches have been proposed in the literature, and our new model to be introduced in Section 3 will draw on some of them. We list some of the known representations of hedonic games below.

We start with a very powerful class of hedonic games that was introduced by Banerjee et al. (2001). An additively separable hedonic game is given by a pair $(A, w)$, where $A=\{1,2, \ldots, n\}$ is a set of players and $w=\left(w_{1}, w_{2}, \ldots, w_{n}\right)$, i.e., each player $i \in A$ has a value function $w_{i}: A \rightarrow \mathbb{R}$ by which she evaluates all players. Now, the players' preferences on coalitions containing them can be

\footnotetext{
${ }^{1}$ While we often stick to the convention that the players' names are numbers (as in $A=\{1,2, \ldots, n\}$ ), we will occasionally deviate from it for the sake of readability.
} 
derived as follows, yielding the corresponding hedonic game $(A, \succeq)$ : For each $i \in A$ and for any two coalitions $B, C \in \mathscr{A}_{i}$, it holds that $B \succeq_{i} C \Longleftrightarrow \sum_{j \in B} w_{i}(j) \geq \sum_{j \in C} w_{i}(j)$.

Dimitrov et al. (2006) introduced a representation that is based on so-called friend- and enemyoriented preference extensions and provides a subclass of the additively separable hedonic games. In their representation, each player $i \in A$ partitions the other players in a set of friends $F_{i} \subseteq A \backslash\{i\}$ and a set of enemies $E_{i}=A \backslash\left(F_{i} \cup\{i\}\right)$, and if $B, C \in \mathscr{A}_{i}$, i's preference over these two coalitions is then determined by the number of friends and enemies she has in them as follows. In the friendoriented preference extension, we define $B \succeq_{i} C$ if and only if $\left|B \cap F_{i}\right|>\left|C \cap F_{i}\right|$ or $\left(\left|B \cap F_{i}\right|=\left|C \cap F_{i}\right|\right.$ and $\left.\left|B \cap E_{i}\right| \leq\left|C \cap E_{i}\right|\right)$, and in the enemy-oriented preference extension, we define $B \succeq_{i} C$ if and only if $\left|B \cap E_{i}\right|<\left|C \cap E_{i}\right|$ or $\left(\left|B \cap E_{i}\right|=\left|C \cap E_{i}\right|\right.$ and $\left.\left|B \cap F_{i}\right| \geq\left|C \cap F_{i}\right|\right)$.

Both encodings can also be represented by additively separable hedonic games. To capture the friend-oriented encoding, each player $i$ has to assign the value $|A|$ to her friends and the value -1 to her enemies. The enemy-oriented encoding results from value functions which assign to each friend the value 1 and to each enemy the value $-|A|$.

A different approach is taken by Cechlárová and Romero-Medina (2001) (see also Cechlárová \& Hajduková, 2003, 2004), who expect the game to be given in the singleton encoding, i.e., each player $i \in A$ has to provide a complete ranking $\unrhd_{i}$ over all players. For any coalition $B \in \mathscr{A}_{i}$, let $\mathscr{B}_{i}(B)$ be any best player $j \in B$ from i's view, i.e., $j \unrhd_{i} k$ for each $k \in B$; and let $\mathscr{W}_{i}(B)=i$ if $B=\{i\}$, and otherwise let $\mathscr{W}_{i}(B)$ be any worst player $j \in B \backslash\{i\}$ from $i$ 's view, i.e., $k \unrhd_{i} j$ for each $k \in B$. Now, for any $B, C \in \mathscr{A}_{i}$, we say $B$ is $\mathscr{B}$-preferred by $i$ over $C$ if $\mathscr{B}_{i}(B) \triangleright_{i} \mathscr{B}_{i}(C)$ or $\left(\mathscr{B}_{i}(B) \sim_{i} \mathscr{B}_{i}(C)\right.$ and $|B|<|C|)$, and we say $B$ is $\mathscr{W}$-preferred by $i$ over $C$ if $\mathscr{W}_{i}(B) \triangleright_{i} \mathscr{W}_{i}(C)$.

\subsection{Stability Concepts}

Important solution concepts for hedonic games are various notions of stability for coalition structures (see, e.g., Bogomolnaia \& Jackson, 2002; Aziz et al., 2013b; Aziz, Brandt, \& Harrenstein, 2013a; Aziz \& Savani, 2016; Elkind \& Rothe, 2015). We focus on concepts that deal with avoiding a player to deviate to another (possibly empty) existing coalition. Relatedly, other commonly studied concepts consider group deviations, such as core stability with the goal that there is no blocking coalition. A third group of stability concepts, such as Pareto optimality and popularity, is based on a relation comparing different coalition structures. For other restrictions of games and other properties, we refer, e.g., to the work of Banerjee et al. (2001).

The following properties are well-known, except for the last one (strict popularity), which is introduced here. A coalition structure $\Gamma$ is called

- perfect if each player $i$ weakly prefers $\Gamma(i)$ to every other coalition containing $i$;

- individually rational if each player $i \in A$ weakly prefers $\Gamma(i)$ to being alone in $\{i\}$;

- Nash stable if for each player $i \in A$ and for each coalition $C \in \Gamma \cup\{\emptyset\}, \Gamma(i) \succeq_{i} C \cup\{i\}$ (that is, no player wants to move to another coalition);

- individually stable if for each player $i \in A$ and for each coalition $C \in \Gamma \cup\{\emptyset\}$, it holds that $\Gamma(i) \succeq_{i} C \cup\{i\}$ or there exists a player $j \in C$ such that $C \succ_{j} C \cup\{i\}$ (that is, no player can move to another coalition without making some player in the new coalition worse off);

- contractually individually stable if for each player $i \in A$ and for each coalition $C \in \Gamma \cup\{\emptyset\}$, it holds that $\Gamma(i) \succeq_{i} C \cup\{i\}$, or there exists a player $j \in C$ such that $C \succ_{j} C \cup\{i\}$, or there exists a player $k \in \Gamma(i) \backslash\{i\}$ such that $\Gamma(i) \succ_{k} \Gamma(i) \backslash\{i\}$ (that is, no player can move to another coalition without making some player in the new coalition or in the old coalition worse off); 
- core stable if for each nonempty coalition $C \subseteq A$, there exists a player $i \in C$ such that $\Gamma(i) \succeq_{i} C$ (that is, no coalition blocks $\Gamma$ );

- strictly core stable if for each nonempty coalition $C \subseteq A$, there exists a player $i \in C$ such that $\Gamma(i) \succ_{i} C$ or for each player $i \in C$, we have $\Gamma(i) \sim_{i} C$ (that is, no coalition weakly blocks $\Gamma$ );

- Pareto optimal if for each coalition structure $\Delta \neq \Gamma$, there exists a player $i \in A$ such that $\Gamma(i) \succ_{i} \Delta(i)$ or for each player $j \in A$, we have $\Gamma(j) \sim_{j} \Delta(j)$ (that is, no other coalition structure Pareto-dominates $\Gamma)$;

- popular if for each coalition structure $\Delta \neq \Gamma$, the number of players $i$ with $\Gamma(i) \succ_{i} \Delta(i)$ is at least as large as the number of players $j$ with $\Delta(j) \succ_{j} \Gamma(j)$;

- strictly popular if for each coalition structure $\Delta \neq \Gamma$, the number of players $i$ with $\Gamma(i) \succ_{i} \Delta(i)$ is larger than the number of players $j$ with $\Delta(j) \succ_{j} \Gamma(j) .^{2}$

\subsection{Complexity Theory}

When studying computational aspects of stability in hedonic games, there are two natural questions that arise. Let $\gamma$ be a stability concept such as those defined in Section 2.2. How hard is it to decide whether a given solution for a given game is $\gamma$-stable and how hard is it for a given game to decide whether there exists a $\gamma$-stable outcome? The former question is the so-called verification variant, which we formally state as follows:

\section{$\gamma$-VERIFICATION}

Given: $\quad$ A hedonic game $H$ and a coalition structure $\Gamma$.

Question: Is $\Gamma$ stable in the sense of $\gamma$ in $H$ ?

The latter question, on the other hand, is referred to as the existence problem, defined as follows:

\section{$\gamma$-EXISTENCE}

Given: A hedonic game $H$.

Question: Is there a coalition structure that is stable in the sense of $\gamma$ in $H$ ?

We assume the reader to be familiar with the complexity classes P and NP. For each stability concept $\gamma$, whenever the problem $\gamma$-VERIFICATION is in P then $\gamma$-EXISTENCE is in NP, by simply guessing a coalition structure and then testing whether it satisfies $\gamma$. There are, however, no further direct connections between these two problems with respect to their complexity. We refer the reader to the interesting and detailed survey by Woeginger (2013a) for further information.

In Section 4, we will show the above problems to be NP-hard for several stability concepts in FEN-hedonic games, and we will do so by reductions from the following well-known NP-complete problems (see Garey \& Johnson, 1979).

\section{EXACT-COVER-BY-THREE-SETS (X3C)}

Given: $\quad$ A set $B=\left\{b_{1}, b_{2}, \ldots, b_{3 m}\right\}, m>1$, and a collection $\mathscr{S}=\left\{S_{1}, S_{2}, \ldots, S_{n}\right\}$ of subsets $S_{i} \subseteq B$ with $\left|S_{i}\right|=3$ for each $i, 1 \leq i \leq n$.

Question: Is there a subcollection $\mathscr{S}^{\prime} \subseteq \mathscr{S}$ such that each element of $B$ occurs in exactly one set in $\mathscr{S}^{\prime}$ ?

\footnotetext{
${ }^{2}$ This notion is adapted from the voting-theoretic term of Condorcet winner: Such a candidate wins an election if and only if she beats each other candidate in pairwise comparison by a (strict) majority of votes.
} 
Note that the problem X3C remains NP-complete even if each element in $B$ occurs in at most three sets in $\mathscr{S}$ (see Garey \& Johnson, 1979).

\begin{tabular}{ll}
\hline & \multicolumn{1}{c}{ CLIQUE } \\
\hline Given: & An undirected graph $G=(V, E)$ and a positive integer $k$. \\
Question: & $\begin{array}{l}\text { Is there a clique (i.e., a subset } V^{\prime} \subseteq V \text { such that each two vertices in } V^{\prime} \text { are } \\
\text { connected by an edge) of size at least } k \text { in } G ?\end{array}$ \\
\hline
\end{tabular}

Beyond that we will encounter problems from the second level of the polynomial hierarchy, namely from $\Sigma_{2}^{p}=\mathrm{NP}^{\mathrm{NP}}$ and $\Pi_{2}^{p}=\operatorname{coNP}^{\mathrm{NP}}$ (see Meyer \& Stockmeyer, 1972; Stockmeyer, 1976). For more background on computational complexity, the reader is referred to the textbooks by Papadimitriou (1995) and Rothe (2005).

\section{Polarized Responsive Preferences and FEN-Hedonic Games}

We now introduce the class of FEN-hedonic games, defined via the following three steps:

1. Similarly to the singleton-encoding and to hedonic games with $\mathscr{W}$-preferences (recall Section 2 for the formal definitions), we start with the assumption that each player $i \in A$ has preferences over the remaining players in $A \backslash\{i\}$. These preferences will be formally defined in Section 3.1 and denoted by $\unrhd_{i}^{+0-}$ for each $i \in A$.

2. To obtain a hedonic game, we have to lift these preferences over players to preferences over coalitions. We will do so by applying a polarized version of the responsive extension principle, which we will formally define in Section 3.2; "PR-extension" in Figure 1 stands for polarized responsive extension.

3. These preferences, denoted by $\succeq_{i}^{+0-}$ for each $i \in A$, can be incomplete in the sense that there might be pairs of coalitions for which $\succeq_{i}^{+0-}$ does not determine which coalition player $i$ prefers. By specifying these missing comparisons, we can extend each $\succeq_{i}^{+0-}$ to complete preferences, which we will collect in the set $\operatorname{Ext}\left(\succeq_{i}^{+0-}\right)$. With these complete preference extensions $\succeq_{i} \in \operatorname{Ext}\left(\succeq_{i}^{+0-}\right)$, we will then define the class of FEN-hedonic games in Section 3.3.

Figure 1 provides an overview of the just described tripartite procedure.

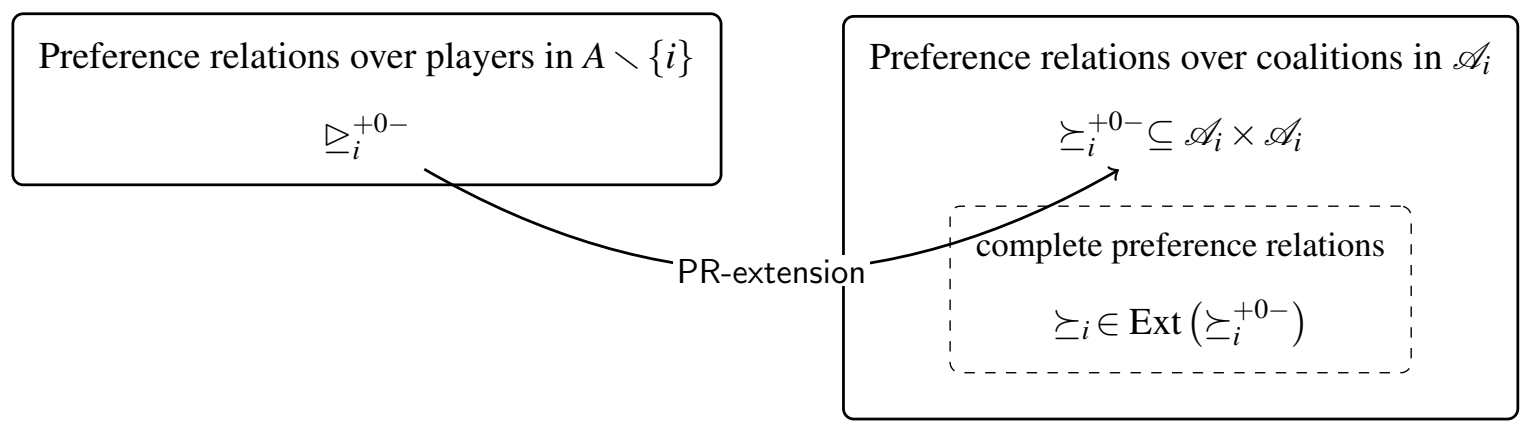

Figure 1: The process of defining the class of FEN-hedonic games for a fixed player $i \in A$ 


\subsection{Preferences over Players: Ordinal Preferences with Double Threshold}

Polarized responsive preferences are a combination of the singleton encoding and the friend- and enemy-oriented encoding with the additional degree of freedom that not all co-players have to be categorized as either friends or enemies. Furthermore, the players have the possibility to provide a ranking of their friends and of their enemies which allows a very fine-grained expression of their opinion. We formalize this intuition in the following definition.

Definition 1 (weak ranking with double threshold) Let $A=\{1,2, \ldots, n\}$ be a set of agents. For each $i \in A$, a weak ranking with double threshold for agent $i$, denoted by $\unrhd_{i}^{+0-}$, consists of $a$ partition of $A \backslash\{i\}$ into three sets:

- $A_{i}^{+}$(i's friends), together with a weak order $\unrhd_{i}^{+}$over $A_{i}^{+}$,

- $A_{i}^{-}$(i's enemies), together with a weak order $\unrhd_{i}^{-}$over $A_{i}^{-}$, and

- $A_{i}^{0}$ (the neutral agents, i.e., the agents $i$ does not care about).

We also write $\unrhd_{i}^{+0-}$ as $\left(\unrhd_{i}^{+}\left|A_{i}^{0}\right| \unrhd_{i}^{-}\right)$. Not having an order of the neutral agents can be interpreted as being indifferent about them all, so it holds that $j \sim_{i} k$ for all $j, k \in A_{i}^{0}$. Furthermore, we assume that each agent $i$ strictly prefers all her friends to her neutral agents, and the neutral players to her enemies. The weak order $\unrhd_{i}$ induced by $\unrhd_{i}^{+0-}$ is therefore defined as follows:

- $\unrhd_{i}$ coincides with $\unrhd_{i}^{+}$on $A_{i}^{+}$;

- $f \triangleright_{i} j$ for each $f \in A_{i}^{+}$and $j \in A_{i}^{0}$;

- $j_{1} \sim_{i} j_{2} \sim_{i} \cdots \sim_{i} j_{k}$ for $A_{i}^{0}=\left\{j_{1}, j_{2}, \ldots, j_{k}\right\}$;

- $j \triangleright_{i} e$ for each $j \in A_{i}^{0}$ and $e \in A_{i}^{-}$; and

- $\unrhd_{i}$ coincides with $\unrhd_{i}^{-}$on $A_{i}^{-}$.

For a set $X=\left\{a_{1}, a_{2}, \ldots, a_{x}\right\} \subseteq A$ in player $i$ 's preference, the shorthand $X_{\sim_{i}}$ denotes that player $i$ is indifferent between all players in $X$, so $a_{1} \sim_{i} a_{2} \sim_{i} \cdots \sim_{i} a_{x}$. Occasionally, we will drop subscript $i$ and simply write $X_{\sim}$ for $X_{\sim_{i}}$ when $i$ is clear from the context. Whenever player $i$ 's set of friends or enemies is empty, we will slightly abuse notation and let $\emptyset$ denote the empty preference $\unrhd_{i}^{+}$or $\unrhd_{i}^{-}$.

Example 2 Let $A=\{1,2, \ldots, 11\}$ and let $\unrhd_{1}^{+0-}=\left(2 \triangleright_{1} 3 \sim_{1} 4|\{5,6,7\}| 8 \triangleright_{1} 9 \sim_{1} 10 \triangleright_{1} 11\right)$ be a weak ranking with double threshold. This means that player 1 likes 2, 3, and 4 (and prefers 2 to both 3 and 4, and is indifferent between 3 and 4); 1 does not care about 5, 6, and 7 (and is indifferent between them); and 1 does not like 8, 9, 10, and 11 (but still prefers 8 to 9 and 10, is indifferent between 9 and 10, and prefers 9 and 10 to 11). The weak order $\unrhd_{1}$ induced by $\unrhd_{1}^{+0-}$ is $2 \triangleright_{1} 3 \sim_{1} 4 \triangleright_{1} 5 \sim_{1} 6 \sim_{1} 7 \triangleright_{1} 8 \triangleright_{1} 9 \sim_{1} 10 \triangleright_{1} 11$.

Note that here the preference between a friend and a neutral player is strict because we assume below that a coalition containing a friend instead of a neutral player is preferred. Analogously, the preference between a neutral player and an enemy is strict because a player does not care about having a neutral player in a coalition but is less happy with having an enemy in the coalition instead.

\subsection{Preferences over Coalitions: Generalizing Responsive Preferences}

Starting from a weak ranking with double threshold $\unrhd_{i}^{+0-}$, which provides a ranking of the players in $A \backslash\{i\}$ from player $i$ 's perspective, we want to deduce player $i$ 's preferences over coalitions 
she is contained in. To do so, we suggest the following generalization of the responsive extension principle.

Definition 3 (extended preference order) Let $\unrhd_{i}^{+0-}$ be a weak ranking with double threshold for agent $i$. The extended preference order $\succeq_{i}^{+0-}$ is defined as follows. For every pair of coalitions $X, Y \in \mathscr{A}_{i}$, we have that $X \succeq_{i}^{+0-} Y$ if and only if the following two conditions hold: (1) There is an injective function $\sigma$ from $Y \cap A_{i}^{+}$to $X \cap A_{i}^{+}$such that for every $y \in Y \cap A_{i}^{+}$, we have $\sigma(y) \unrhd_{i} y$. (2) There is an injective function $\theta$ from $X \cap A_{i}^{-}$to $Y \cap A_{i}^{-}$such that for every $x \in X \cap A_{i}^{-}$, we have $x \unrhd_{i} \theta(x)$. Finally, $X \succ_{i}^{+0-} Y$ if and only if $X \succeq_{i}^{+0-} Y$ and not $Y \succeq_{i}^{+0-} X$, and $X \sim^{+0-} Y$ if and only if $X \succeq^{+0-} Y$ and $Y \succeq^{+0-} X$.

Intuitively speaking, for a fixed coalition, adding a further friend makes the coalition strictly more valuable, while adding an enemy causes the opposite. When exchanging two friends, the valuation of the coalition changes depending on the relation between the exchanged players (the same holds when two enemies are exchanged). When both a friend and an enemy are added or when they both are removed, the original and the new coalition are incomparable with respect to the responsive extension principle.

Thus, to construct the polarized responsive extension (PR-extension, for short) for a player $i$, we start with the coalition consisting of $i$ and her friends (which is $i$ 's most preferred coalition) and then construct all directly comparable coalitions by adding enemies, removing friends, or exchanging enemies or friends. For each newly obtained coalition, we repeat this procedure until we reach $i$ 's least preferred coalition consisting of $i$ and all of $i$ 's enemies. Note that the elements of $A_{i}^{0}$ are disregarded, as their addition to or removal from a coalition does not change the coalition's value for $i$. The following examples illustrate the just presented extension principle.

Example 4 For $A=\{1,2, \ldots, 6\}$, consider the weak ranking with double threshold of player 1 given by $\unrhd_{1}^{+0-}=\left(2 \triangleright_{1} 3 \sim_{1} 4|\emptyset| 5 \triangleright_{1} 6\right)$. The graph in Figure 2 shows the partial order obtained from the polarized responsive extension of this preference, where an arc from coalition $X$ to coalition $Y$ implies that $X \succ_{1}^{+0-} Y$. Hence, any path leading from $X^{\prime}$ to $Y^{\prime}$ implies $X^{\prime} \succ_{1}^{+0-} Y^{\prime}$, whereas coalitions that are not connected by a path, such as $\{1,2,3\}$ and $\{1,2,3,4,5\}$, are incomparable. Note that if there were additional players $j>6$ in A considered as neutral by player 1 , the general picture would be the same with additional indifferences between any $C \subseteq\{2, \ldots, 6\}$ and $\{1\} \cup C \cup N$ for any $N \subseteq A \backslash\{1, \ldots, 6\}$. These indifferences would occur at each level and for each coalition.

Example 5 For $A=\{1,2,3,4,5\}$, let the first player's preferences be $\unrhd_{1}^{+0-}=\left(2 \triangleright_{1} 3|\emptyset| 4 \triangleright_{1} 5\right)$. The graph in Figure 3 shows the partial order obtained from the polarized responsive extension of this preference using the same notation as in Example 4.

Intuitively, the relation between two coalitions $C$ and $D\left(C \succ_{i}^{+0-} D\right.$, or $D \succ_{i}^{+0-} C$, or $C \sim_{i}^{+0-} D$, or $C$ and $D$ are incomparable) from player $i$ 's point of view can be determined by the characterization given in Proposition 6, which is inspired by the work of Aziz et al. (2015) and of Bouveret et al. (2010) who show characterizations for the original responsive order in the context of fair division. Essentially, the characterization of Proposition 6 tells us how the extended order $\succeq_{i}^{+0-}$ induced by a weak ranking with double threshold $\unrhd_{i}^{+0-}$ for agent $i$ depends on the number of $i$ 's friends and enemies in the coalitions and on the ranking of, respectively, friends and enemies contained in them. 
Kerkmann, LAng, Rey, Rothe, Schadrack \& SCHEnd

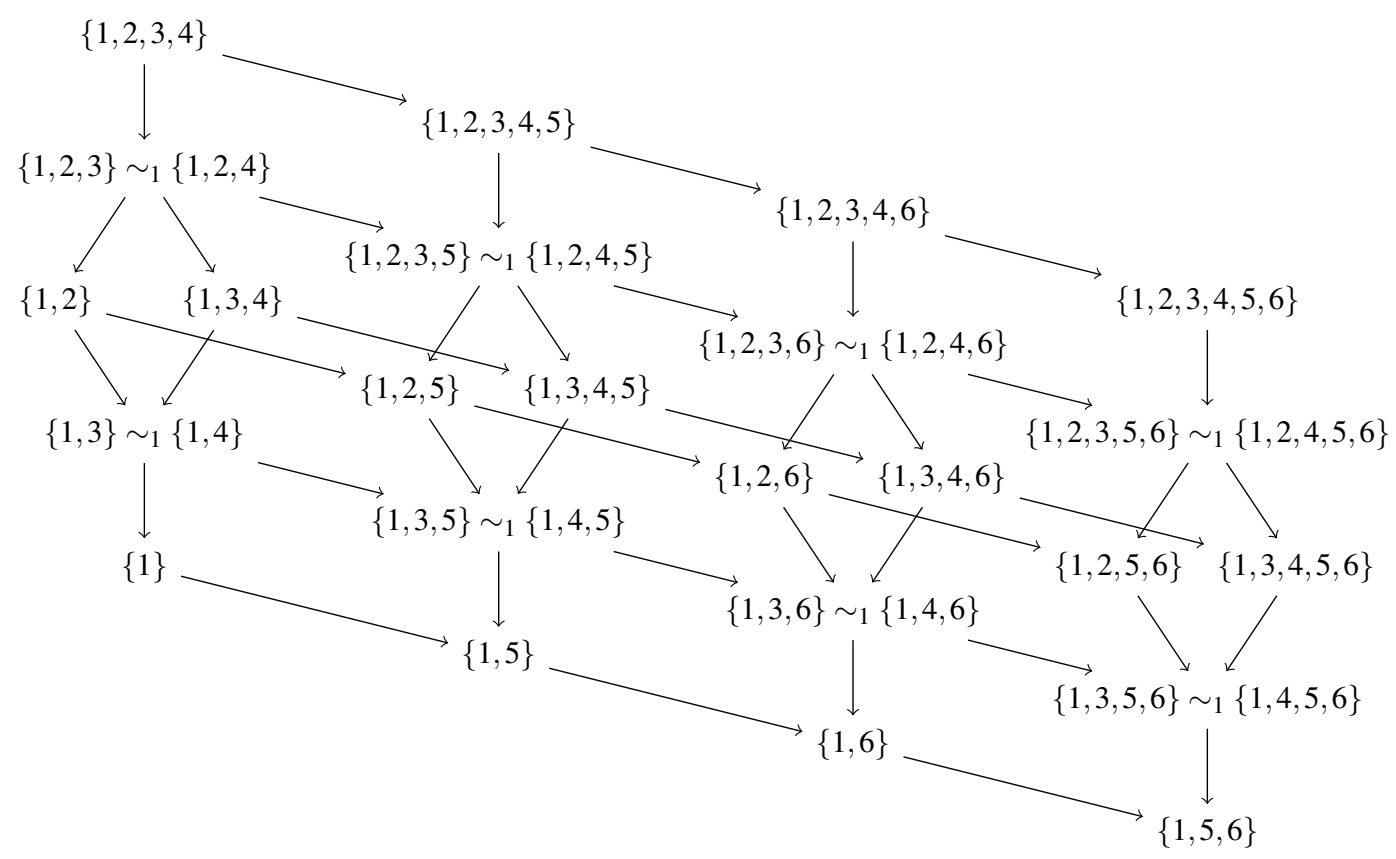

Figure 2: Partial order from the polarized responsive extension of $\unrhd_{1}^{+0-}=\left(2 \triangleright_{1} 3 \sim_{1} 4|\emptyset| 5 \triangleright_{1} 6\right)$

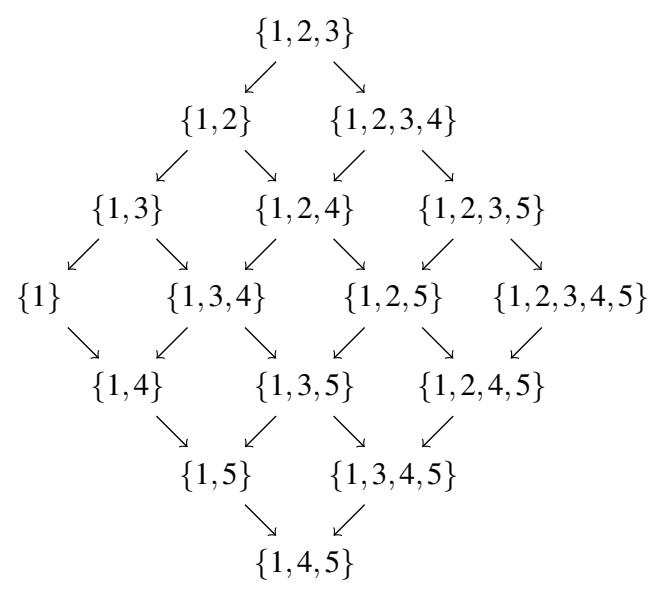

Figure 3: Partial order from the polarized responsive order of $\unrhd_{1}^{+0-}=\left(2 \triangleright_{1} 3|\emptyset| 4 \triangleright_{1} 5\right)$

Proposition 6 Let $\unrhd_{i}^{+0-}$ be a weak ranking with double threshold for agent $i$, and let $C$ and $D$ be any two coalitions containing $i$. Consider the orders $f_{1} \unrhd_{i} f_{2} \unrhd_{i} \cdots \unrhd_{i} f_{\mu}$ with $\left\{f_{1}, f_{2}, \ldots, f_{\mu}\right\}=$ $C \cap A_{i}^{+}$and $f_{1}^{\prime} \unrhd_{i} f_{2}^{\prime} \unrhd_{i} \cdots \unrhd_{i} f_{\mu^{\prime}}^{\prime}$ with $\left\{f_{1}^{\prime}, f_{2}^{\prime}, \ldots, f_{\mu^{\prime}}^{\prime}\right\}=D \cap A_{i}^{+}$, as well as $e_{1} \unrhd_{i} e_{2} \unrhd_{i} \cdots \unrhd_{i} e_{v}$ with $\left\{e_{1}, e_{2}, \ldots, e_{v}\right\}=C \cap A_{i}^{-}$and $e_{1}^{\prime} \unrhd_{i} e_{2}^{\prime} \unrhd_{i} \ldots \unrhd_{i} e_{v^{\prime}}^{\prime}$ with $\left\{e_{1}^{\prime}, e_{2}^{\prime}, \ldots, e_{v^{\prime}}^{\prime}\right\}=D \cap A_{i}^{-}$. Then $C \succeq_{i}^{+0-} D$ if and only if

(a) $\mu \geq \mu^{\prime}$ and $v \leq v^{\prime}$, 
(b) for each $k, 1 \leq k \leq \mu^{\prime}$, it holds that $f_{k} \unrhd_{i} f_{k}^{\prime}$, and

(c) for each $\ell, 1 \leq \ell \leq v$, it holds that $e_{v-\ell+1} \unrhd_{i} e_{v^{\prime}-\ell+1}^{\prime}$.

Proof. If $(a)$ to $(c)$ hold, the two injective functions $\sigma: D \cap A_{i}^{+} \rightarrow C \cap A_{i}^{+}$and $\theta: C \cap A_{i}^{-} \rightarrow D \cap A_{i}^{-}$ mapping $f_{k}^{\prime} \mapsto f_{k}$ for each $k, 1 \leq k \leq \mu^{\prime}$, and $e_{v-\ell+1} \mapsto e_{v^{\prime}-\ell+1}^{\prime}$ for each $\ell, 1 \leq \ell \leq v$, satisfy $\sigma\left(f_{k}^{\prime}\right) \unrhd_{i} f_{k}^{\prime}$ and $e_{v-\ell+1} \unrhd_{i} \theta\left(e_{v-\ell+1}\right)$, for the same range of $k$ and $\ell$. On the other hand, if there are two injective functions with the desired requirements, $(a)$ holds. If there were some $k$ with $f_{k}^{\prime} \triangleright_{i} f_{k}$ (or some $\ell$ with $e_{v^{\prime}-\ell+1}^{\prime} \triangleright_{i} e_{v-\ell+1}$ ), this would imply $\sigma\left(f_{k}^{\prime}\right)=f_{j}$ for some $j<k$ (respectively, $\theta\left(e_{v-\ell+1}\right)=e_{v-j+1}^{\prime}$ for some $\left.j>\ell\right)$. This, however, implies that either a requirement is violated for $f_{1}^{\prime}$ (or $e_{v}$ ), or that $\sigma$ (or $\theta$ ) is not injective, a contradiction.

\subsection{The Class of FEN-Hedonic Games}

Now we define hedonic games where each player has friends, enemies, and neutral co-players, and preferences over the former two sets such that we can derive each player's preference relation as introduced in the previous section. We call them FEN-hedonic games and define them formally as follows.

Definition 7 (FEN-hedonic game) $A$ FEN-hedonic game is a pair $H=\left(A,\left(\unrhd_{1}^{+0-}, \ldots, \unrhd_{n}^{+0-}\right)\right)$, where $A=\{1,2, \ldots, n\}$ is a set of players, and $\unrhd_{i}^{+0-}$ gives the weak ranking with double threshold of player $i \in A$ as defined in Definition 1 .

To obtain the players' preferences over coalitions, we use the polarized responsive extension that we defined in Section 3.2. Since these preference relations $\succeq_{i}^{+0-}$ can be incomplete, we consider their extensions to complete relations, which have to preserve both already defined strict comparisons and indifferences.

Definition 8 (possible and necessary (weak) preference) A preference relation $\succeq_{i}$ over $\mathscr{A}_{i}$ extends $\succeq_{i}^{+0-}$ if (1) $C \succ_{i}^{+0-} D$ implies $C \succ_{i} D$ for all coalitions $C, D \in \mathscr{A}_{i}$; and (2) $C \sim_{i}^{+0-} D$ implies $C \sim_{i} D$ for all coalitions $C, D \in \mathscr{A}_{i}$.

Let $\operatorname{Ext}\left(\succeq_{i}^{+0-}\right)$ be the set of all complete preference relations extending $\succeq_{i}^{+0-}$. We say

- $i$ possibly weakly prefers $C$ to $D$ if $C \succeq_{i} D$ for some $\succeq_{i} \in \operatorname{Ext}\left(\succeq_{i}^{+0-}\right)$;

- $i$ possibly prefers $C$ to $D$ if $C \succ_{i} D$ (i.e., $C \succeq_{i} D$ and not $D \succeq_{i} C$ ) for some $\succeq_{i} \in \operatorname{Ext}\left(\succeq_{i}^{+0-}\right)$;

- $i$ necessarily weakly prefers $C$ to $D$ if $C \succeq_{i} D$ for all $\succeq_{i} \in \operatorname{Ext}\left(\succeq_{i}^{+0-}\right)$; and

- $i$ necessarily prefers $C$ to $D$ if $C \succ_{i} D$ (i.e., $C \succeq_{i} D$ and not $D \succeq_{i} C$ ) for all $\succeq_{i} \in \operatorname{Ext}\left(\succeq_{i}^{+0-}\right)$.

Equivalently, the above definitions can also be formulated as follows: $i$ possibly weakly prefers $C$ to $D$ if $C \succeq_{i}^{+0-} D$ or $C$ and $D$ are incomparable with respect to $\succeq^{+0-}$; $i$ possibly prefers $C$ to $D$ if $C \succ_{i}^{+0-} D$ or $C$ and $D$ are incomparable with respect to $\succeq^{+0-}$; $i$ necessarily weakly prefers $C$ to $D$ if $C \succeq_{i}^{+0-} D$; and $i$ necessarily prefers $C$ to $D$ if $C \succ_{i}^{+0-} D$.

We will see that weak rankings with double threshold can have various complete extensions. 
Example 9 Consider the FEN-hedonic game whose players in $A=\{1,2,3\}$ have the following weak rankings with double threshold: $\unrhd_{1}^{+0-}=\left(2 \triangleright_{1} 3|\emptyset| \emptyset\right), \unrhd_{2}^{+0-}=(3|\emptyset| 1)$, and $\unrhd_{3}^{+0-}=$ $(1|\{2\}| \emptyset)$. The polarized responsive orders are

$$
\{1,2,3\} \succ_{1}^{+0-}\{1,2\} \succ_{1}^{+0-}\{1,3\} \succ_{1}^{+0-}\{1\}
$$

for player 1 ,

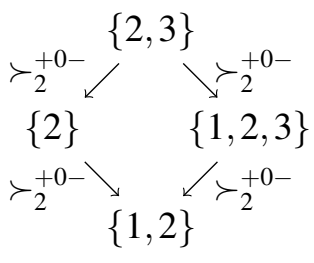

for player 2 , and

$$
\{1,3\} \sim_{3}^{+0-}\{1,2,3\} \succ_{3}^{+0-}\{3\} \sim_{3}^{+0-}\{2,3\}
$$

for player 3. So, two preferences are already complete, and there are three complete preferences extending $\succeq_{2}^{+0-}$, one setting $\{2\} \succ_{2}\{1,2,3\}$, another setting $\{2\} \sim_{2}\{1,2,3\}$, and the third setting $\{1,2,3\} \succ_{2}\{2\}$, leaving all other relations the same.

\subsection{Optimistic and Pessimistic Preference Extensions}

We will now introduce two (generally incomplete) preference extensions. For a given coalition $C$ containing player $i$, we consider the optimistic extension $\succeq_{i}^{+C}$ of player $i$ 's preference which ranks $C$ as high as possible, and the pessimistic extension $\succeq_{i}^{-C}$ which ranks $C$ as low as possible. We will make intensive use of these extensions for stating characterizations of stability in FEN-hedonic games, considering the stability concepts defined in Section 2.2, and for deriving polynomial-time algorithms in Section 4.

Definition 10 (optimistic and pessimistic extension) Let $C \in \mathscr{A}_{i}$ be a coalition containing player $i \in A$ and $\succeq_{i}^{+0-}$ be i's preference relation. We define the following two relations:

- $R_{i}^{+C}=\succeq_{i}^{+0-} \cup\left\{\left(C, C^{\prime}\right) \mid C^{\prime} \nsucc_{i}^{+0-} C, C^{\prime} \in \mathscr{A}_{i}\right\}$ and

- $R_{i}^{-C}=\succeq_{i}^{+0-} \cup\left\{\left(C^{\prime}, C\right) \mid C \nsucc_{i}^{+0-} C^{\prime}, C^{\prime} \in \mathscr{A}_{i}\right\}$.

Let $\succeq_{i}^{+C}$ be the transitive closure of $R_{i}^{+C}$ and $\succeq_{i}^{-C}$ be the transitive closure of $R_{i}^{-C}$. The strict preference relations $\succ_{i}^{+C}$ and $\succ_{i}^{-C}$ and indifference relations $\sim_{i}^{+C}$ and $\sim_{i}^{-C}$ are defined as usual.

Note that $\succeq_{i}^{+C}$ and $\succeq_{i}^{-C}$ extend $\succeq_{i}^{+0-}$ where $\succeq_{i}^{+C}$ ranks $C$ as high as possible and $\succeq_{i}^{-C}$ ranks $C$ as low as possible. Therefore, we call $\succeq_{i}^{+C}$ the optimistic and $\succeq_{i}^{-C}$ the pessimistic extension of $\succeq_{i}^{+0-}$ with respect to $C \in \mathscr{A}_{i}$.

The following characterization follows directly from the definitions of $R_{i}^{+C}$ and $R_{i}^{-C}$.

Observation 11 Consider any $C, D, E \in \mathscr{A}_{i}$.

(1) $(D, E) \in R_{i}^{+C}$ if and only if $D \succeq_{i}^{+0-} E$ or $E \nsucc_{i}^{+0-} D=C$. 

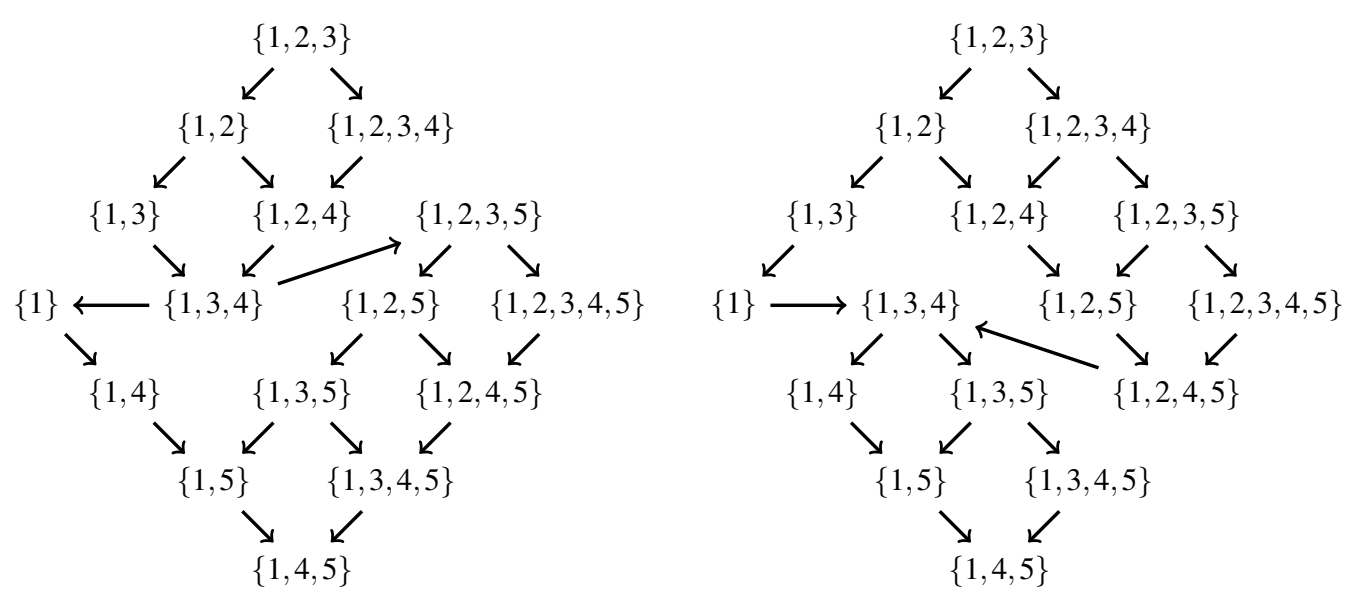

Figure 4: Optimistic extension $\succeq_{1}^{+\{1,3,4\}}$ (left) and pessimistic extension $\succeq_{1}^{-\{1,3,4\}}$ (right) of $\succeq_{1}^{+0-}$ from Example 5

(2) $(D, E) \in R_{i}^{-C}$ if and only if $D \succeq_{i}^{+0-} E$ or $C=E \nsucc_{i}^{+0-} D$.

Example 12 Consider the FEN-hedonic game from Example 5 and let $C=\{1,3,4\}$.

The relations $\succeq_{1}^{+C}$ and $\succeq_{1}^{-C}$ are shown in Figure 4 where all arrows induce transitivity. As usual, we do not show the edges induced by reflexivity and transitivity.

Proposition 13 Let $C, D, E \in \mathscr{A}_{i}$.

(1) $D \succeq_{i}^{+C} E$ if and only if $(D, E) \in R_{i}^{+C}$ or $(D, C),(C, E) \in R_{i}^{+C}$.

(2) $D \succeq_{i}^{-C} E$ if and only if $(D, E) \in R_{i}^{-C}$ or $(D, C),(C, E) \in R_{i}^{-C}$.

Proof. We only show (1); the proof for (2) is similar. If $C=D$ or $D=E$ or $C=E$, then (1) is obvious, so we asusme now that $C, D$ and $E$ are all different.

The implication from right to left is obvious.

To prove the implication from left to right, we show that $D \succeq_{i}^{+C} E$ and $(D, E) \notin R_{i}^{+C}$ imply $(D, C),(C, E) \in R_{i}^{+C}$. Assume that (a) $D \succeq_{i}^{+C} E$ and (b) $(D, E) \notin R_{i}^{+C}$. From (a) and the definition of $\succeq_{i}^{+C}$ as the transitive closure of $R_{i}^{+C}$, there are $m \geq 1$ and $C_{1}, \ldots, C_{m} \in \mathscr{A}_{i}$ such that (c) $\left(D, C_{1}\right),\left(C_{1}, C_{2}\right), \ldots,\left(C_{m}, E\right) \in R_{i}^{+C}$.

By taking the smallest such $m$, there is at most one $j \in\{1, \ldots, m\}$ with $C=C_{j}$. Now, assume that $C \neq C_{j}$ for all $j \in\{1, \ldots, m\}$. Because for $X \neq C,(X, Y) \in R_{i}^{+C}$ is equivalent to $X \succeq_{i}^{+0-} Y$, we have $D \succeq_{i}^{+0-} C_{1} \succeq_{i}^{+0-} C_{2} \succeq_{i}^{+0-} \ldots \succeq_{i}^{+0-} C_{m} \succeq_{i}^{+0-} E$, hence $D \succeq_{i}^{+0-} E$, contradicting (b). Therefore, there must be exactly one $j \in\{1, \ldots, m\}$ such that $C=C_{j}$.

If $j=1$, then from (c) we directly get $(D, C) \in R_{i}^{+C}$. Since for $X \neq C,(X, Y) \in R_{i}^{+C}$ is equivalent to $X \succeq_{i}^{+0-} Y$, we furthermore get $C \succeq_{i}^{+0-} C_{2} \succeq_{i}^{+0-} \ldots \succeq_{i}^{+0-} C_{m} \succeq_{i}^{+0-} E$, hence $C \succeq_{i}^{+0-} E$, which implies $(C, E) \in R_{i}^{+C}$. Analogously, if $j=m$, we directly get $(C, E) \in R_{i}^{+C}$ and $D \succeq_{i}^{+0-} C_{1} \succeq_{i}^{+0-}$ $C_{2} \succeq_{i}^{+0-} \ldots \succeq_{i}^{+0-} C_{m-1} \succeq_{i}^{+0-} C$, which implies $(D, C) \in R_{i}^{+C}$. If $1<j<m$, then (c) implies (d) $D \succeq_{i}^{+0-} C$, (e) $C_{j+1} \succeq_{i}^{+0-} C$, and (f) $C_{j+1} \succeq_{i}^{+0-} E$. (d) implies $(D, C) \in R_{i}^{+C}$. Assume now 
that $(C, E) \notin R_{i}^{+C}$, which is equivalent to $E \succ_{i}^{+0-} C$; together with (f) this implies $C_{j+1} \succ_{i}^{+0-} C$, contradicting (e).

\section{Proposition 14 Consider any $C, D \in \mathscr{A}_{i}$.}

(1) $C \succeq_{i}^{+C} D$ if and only if $(C, D) \in R_{i}^{+C}$, which in turn holds if and only if $D \nsucc_{i}^{+0-} C$.

(2) $D \succeq_{i}^{+C} C$ if and only if $(D, C) \in R_{i}^{+C}$, which in turn holds if and only if $D \succeq_{i}^{+0-} C$.

(3) $C \succeq_{i}^{-C} D$ if and only if $(C, D) \in R_{i}^{-C}$, which in turn holds if and only if $C \succeq_{i}^{+0-} D$.

(4) $D \succeq_{i}^{-C} C$ if and only if $(D, C) \in R_{i}^{-C}$, which in turn holds if and only if $C \nsucc_{i}^{+0-} D$.

Proof. For each point the first equivalence follows by applying Proposition 13 and the second equivalence follows by applying Observation 11.

Combining Observation 11, Proposition 13, and Proposition 14, we furthermore get:

\section{Proposition 15 Consider any $C, D, E \in \mathscr{A}_{i}$.}

(1) $D \succeq_{i}^{+C} E$ if and only if $D \succeq_{i}^{+0-} E$ or $\left(D \succeq_{i}^{+0-} C\right.$ and $\left.E \nsucc_{i}^{+0-} C\right)$.

(2) $D \succeq_{i}^{-C} E$ if and only if $D \succeq_{i}^{+0-} E$ or $\left(C \nsucc_{i}^{+0-} D\right.$ and $\left.C \succeq_{i}^{+0-} E\right)$.

Proof. We only show (1). (2) can be shown similarly.

By Proposition 13, we have $D \succeq_{i}^{+C} E$ if and only if $(D, E) \in R_{i}^{+C}$ or $(D, C),(C, E) \in R_{i}^{+C}$. By Observation 11, this is equivalent to $D \succeq_{i}^{+0-} E$ or $E \nsucc_{i}^{+0-} D=C$ or $(D, C),(C, E) \in R_{i}^{+C}$. By Proposition 14 , this in turn is equivalent to $D \succeq_{i}^{+0-} E$ or $E \nsucc_{i}^{+0-} D=C$ or $\left(D \succeq_{i}^{+0-} C\right.$ and $\left.E \nsucc_{i}^{+0-} C\right)$. Since $E \nsucc_{i}^{+0-} D=C$ implies $\left(D \succeq_{i}^{+0-} C\right.$ and $\left.E \nsucc_{i}^{+0-} C\right)$, the condition can be shortened to $D \succeq_{i}^{+0-} E$ or $\left(D \succeq_{i}^{+0-} C\right.$ and $\left.E \nsucc_{i}^{+0-} C\right)$.

Proposition 16 The strict relations $\succ_{i}^{+C}$ and $\succ_{i}^{-C}$ are acyclic.

Proof. We give the proof for $\succ_{i}^{+C}$ only. The proof for $\succ_{i}^{-C}$ is similar.

Suppose that $\succ_{i}^{+C}$ contains a cycle $C_{1} \succ_{i}^{+C} C_{2} \succ_{i}^{+C} \cdots \succ_{i}^{+C} C_{q} \succ_{i}^{+C} C_{1}$. Because $\succeq_{i}^{+C}$ contains $\succ_{i}^{+C}$ and $\succeq_{i}^{+C}$ is the transitive closure of $R_{i}^{+C}, R_{i}^{+C}$ contains a cycle of the form $C_{1}^{\prime}, C_{2}^{\prime}, \ldots, C_{k}^{\prime}, C_{1}^{\prime}$, with $C_{1}^{\prime}=C_{1}$ and $\left\{C_{2}, \ldots, C_{q}\right\} \subseteq\left\{C_{2}^{\prime}, \ldots, C_{k}^{\prime}\right\}$; in particular, $C_{2}=C_{r}^{\prime}$ for some $r \in\{2, \ldots, k\}$.

If $C \neq C_{j}^{\prime}$ for all $j \in\{1, \ldots, k\}$, then because for $X \neq C,(X, Y) \in R_{i}^{+C}$ is equivalent to $X \succeq_{i}^{+0-} Y$, we have that $C_{1} \sim_{i}^{+0-} C_{2}^{\prime} \sim_{i}^{+0-} \ldots \sim_{i}^{+0-} C_{k}^{\prime}$, thus $C_{1} \sim_{i}^{+0-} C_{r}^{\prime}$, contradicting $C_{1} \succ_{i}^{+C} C_{2}$.

Therefore, $C=C_{j}^{\prime}$ and $C_{j}^{\prime} \nsucceq_{i}^{+0-} C_{j+1}^{\prime}$ for some $j \in\{1, \ldots, k\}$. Without loss of generality, let $j=1$ be the only index with $C=C_{j}^{\prime}$. Then $\left(C, C_{2}^{\prime}\right), \ldots,\left(C_{k}^{\prime}, C\right) \in R_{i}^{+C}$ implies $C \nsucceq_{i}^{+0-} C_{2}^{\prime}$ and $C_{2}^{\prime} \succeq_{i}^{+0-} \cdots \succeq_{i}^{+0-} C_{k}^{\prime} \succeq_{i}^{+0-} C$; however, by Proposition $14,\left(C, C_{2}^{\prime}\right) \in R_{i}^{+C}$ is equivalent to $C_{2}^{\prime} \nsucc_{i}^{+0-}$ $C$, a contradiction.

Proposition $17 \succeq_{i}^{+C}$ and $\succeq_{i}^{-C}$ are extensions of $\succeq_{i}^{+0-}$ with respect to Definition 8 . 
Proof. We show that (1) and (2) from Definition 8 hold for $\succeq_{i}^{+C}$. The proof for $\succeq_{i}^{-C}$ is similar.

(1) Let $D \succ_{i}^{+0-} E$ for two coalitions $D, E \in \mathscr{A}_{i}$. This means that $D \succeq_{i}^{+0-} E$ and $E \succeq_{i}^{+0-} D$. By Proposition 15, $D \succeq_{i}^{+0-} E$ implies $D \succeq_{i}^{+C} E$. Assume, for the sake of contradiction, that $E \succeq_{i}^{+C} D$. Then, by Proposition 15, we have $E \succeq_{i}^{+0-} D$ or $\left(E \succeq_{i}^{+0-} C\right.$ and $\left.D \nsucc_{i}^{+0-} C\right)$. However, $E \succeq_{i}^{+0-} D$ does not hold because of $D \succ_{i}^{+0-} E$. $\left(E \succeq_{i}^{+0-} C\right.$ and $\left.D \nsucc_{i}^{+0-} C\right)$ does not hold either because $D \succ_{i}^{+0-} E \succeq_{i}^{+0-} C$ contradicts $D \nsucc_{i}^{+0-} C$. Hence, the assumption was false and $E \nsucceq_{i}^{+C} D$. With $D \succeq_{i}^{+C} E$ we have $D \succ_{i}^{+C} E$.

(2) Let $D \sim_{i}^{+0-} E$ for two coalitions $D, E \in \mathscr{A}_{i}$. This means that $D \succeq_{i}^{+0-} E$ and $E \succeq_{i}^{+0-} D$. Then, by Proposition 15, $D \succeq_{i}^{+0-} E$ implies $D \succeq_{i}^{+C} E$ and $E \succeq_{i}^{+0-} D$ implies $E \succeq_{i}^{+C} D$. Hence, $D \sim_{i}^{+C} E$.

We furthermore observe that $\succeq_{i}^{+C}$ and $\succeq_{i}^{-C}$ are never undecided concerning $C$.

Observation 18 For any two coalitions $C, D \in \mathscr{A}_{i}$, it holds that $C \succeq_{i}^{+C} D$ or $D \succeq_{i}^{+C} C$ and that $C \succeq_{i}^{-C} D$ or $D \succeq_{i}^{-C} C$.

Proof. First, assume that $C \nsucceq_{i}^{+C} D$ and $D \nsucceq_{i}^{+C} C$. It follows by Proposition 14 that $D \succ_{i}^{+0-} C$ and that $D \nsucceq_{i}^{+0-} C$. This is a contradiction. Similarly, assume that $C \nsucceq_{i}^{-C} D$ and $D \nsucceq_{i}^{-C} C$. It then follows by Proposition 14 that $C \Varangle_{i}^{+0-} D$ and $C \succ_{i}^{+0-} D$, which again is a contradiction.

Proposition 19 Consider any $C, D \in \mathscr{A}_{i}$.

1. There exists a complete extension $\succeq_{i}$ of $\succeq_{i}^{+0-}$ satisfying

(a) $C \succeq_{i} D$ if and only if $C \succeq_{i}^{+C} D$.

(b) $C \succ_{i} D$ if and only if $C \succ_{i}^{+C} D$.

(c) $D \succeq_{i} C$ if and only if $D \succeq_{i}^{-C} C$.

(d) $D \succ_{i} C$ if and only if $D \succ_{i}^{-C} C$.

2. All complete extensions $\succeq_{i}$ of $\succeq_{i}^{+0-}$ satisfy

(a) $C \succeq_{i} D$ if and only if $C \succeq_{i}^{-C} D$.

(b) $C \succ_{i} D$ if and only if $C \succ_{i}^{-C} D$.

(c) $D \succeq_{i} C$ if and only if $D \succeq_{i}^{+C} C$.

(d) $D \succ_{i} C$ if and only if $D \succ_{i}^{+C} C$.

Proof. We only prove points 1(a) and (2)b. The proofs of all other points are similar.

First consider 1(a). From left to right, assume that $C \succeq_{i}^{+C} D$ does not hold. By Proposition 14, this implies $D \succ_{i}^{+0-} C$. Hence, every extension $\succeq_{i}$ of $\succeq_{i}^{+0-}$ satisfies $D \succ_{i} C$, which implies $C \succeq_{i} D$. From right to left, assume that $C \succeq_{i}^{+C} D$. Since $\succ_{i}^{+C}$ is acyclic by Proposition 16, $\succeq_{i}^{+C}$ can be extended to a complete preference relation. Consider such a complete extension $\succeq_{i}$ of $\succeq_{i}^{+C}$. It then holds by definition of the responsive extension that $C \succeq_{i} D$. Furthermore, since $\succeq_{i}^{+C}$ is an extension of $\succeq_{i}^{+0-}$ (and $\succeq_{i}$ is an extension of $\succeq_{i}^{+C}$ ), $\succeq_{i}$ is also an extension of $\succeq_{i}^{+0-}$.

Now consider 2(b). From left to right, assume that for all $\succeq_{i} \in \operatorname{Ext}\left(\succeq_{i}^{+0-}\right)$ we have $C \succ_{i} D$. Since $\succ_{i}^{-C}$ is acyclic by Proposition 16, $\succeq_{i}^{-C}$ can be extended to a complete preference relation. 
Consider such an extension $\succeq_{i} \in \operatorname{Ext}\left(\succeq_{i}^{-C}\right)$. Then $\succeq_{i}$ is also an extension of $\succeq_{i}^{+0-}$. Hence, $C \succ_{i} D$ holds, which in turn means that $C \swarrow_{i} D$. Since $\succeq_{i}$ is an extension of $\succeq_{i}^{-C}$, it follows that $C \npreceq_{i}^{+C} D$. Since $\succeq_{i}^{-C}$ is never undecided concerning $C$ (see Observation 18), it follows that $C \succ_{i}^{-C} D$. From right to left, assume that $C \succ_{i}^{-C} D$, i.e., $C \succeq_{i}^{-C} D$ and $D \succeq_{i}^{-C} C$. With Proposition 14(3) the latter implies $C \succ_{i}^{+0-} D$. Hence, for every extension $\succeq_{i}$ of $\succeq_{i}^{+0-}$, it holds that $C \succ_{i} D$.

Finally, note that whether $(D, E) \in R_{i}^{+C}$ or $(D, E) \in R_{i}^{-C}$ holds for any three coalitions $C, D, E \in$ $\mathscr{A}_{i}$ can be decided in polynomial time, since $D \succeq_{i}^{+0-} E$ and $D \succeq_{i}^{+0-} E$ can be decided in polynomial time by Proposition 6. Furthermore, $D \succeq_{i}^{+C} E$ and $D \succeq_{i}^{-C} E$ can be decided in polynomial time by Proposition 15.

\section{Stability in FEN-Hedonic Games}

Now that we have defined our new games and have stated some axiomatic properties, we turn to the complexity of verifying stable outcomes or checking whether there exists one. We have seen that the preference extensions obtained by applying the polarized responsive extension principle to the players' weak rankings with double threshold can lead to incomplete preferences over coalitions. In this section, we consider one possibility to deal with these incomparabilities: We leave them open and consider every possible extension and then study the complexity of related decision problems.

\subsection{Possible and Necessary Stability: Properties and Characterizations}

We start with formally defining the notions of possible and necessary stability for games with incomplete preference extensions.

Definition 20 (possible and necessary stability) Let $\gamma$ be a stability concept for hedonic games, $\left(A,\left(\unrhd_{1}^{+0-}, \ldots, \unrhd_{n}^{+0-}\right)\right)$ be a FEN-hedonic game, and $\Gamma$ be a coalition structure. $\Gamma$ is said to be possibly $\gamma$ if there exists a profile $\left(\succeq_{1}, \ldots, \succeq_{n}\right)$ in $\times_{i=1}^{n} \operatorname{Ext}\left(\succeq_{i}^{+0-}\right)$ such that $\Gamma$ satisfies $\gamma$ in $\left(A,\left(\succeq_{1}\right.\right.$ $\left.\left., \ldots, \succeq_{n}\right)\right)$. $\Gamma$ is said to be necessarily $\gamma$ iffor each $\left(\succeq_{1}, \ldots, \succeq_{n}\right)$ in $\times_{i=1}^{n} \operatorname{Ext}\left(\succeq_{i}^{+0-}\right)$, $\Gamma$ satisfies $\gamma$ in $\left(A,\left(\succeq_{1}, \ldots, \succeq_{n}\right)\right)$.

Observe first that there always is a necessarily individually rational coalition structure (namely, the coalition structure where every agent is alone). For each extension, there exists a Pareto optimal coalition structure (where different extensions may have different Pareto optimal coalition structures) so that there is always a possibly Pareto optimal coalition structure (see Theorem 30 in Section 4.4 for a formal proof). We can furthermore state the following characterizations for possible/necessary perfectness and individual rationality.

Proposition 21 Consider a FEN-hedonic game $\left(A,\left(\unrhd_{1}^{+0-}, \ldots, \unrhd_{n}^{+0-}\right)\right)$ and a coalition structure $\Gamma$.

1. $\Gamma$ satisfies necessary perfectness if and only if it satisfies possible perfectness, and both statements are equivalent to the following condition: For each player $i, A_{i}^{+} \subseteq \Gamma(i)$ and $A_{i}^{-} \cap \Gamma(i)=\emptyset$, that is, all friends of $i$ are in her coalition, and none of her enemies is.

2. $\Gamma$ is possibly individually rational if and only if for each $i \in A, \Gamma(i)$ contains at least a friend of i's or no enemies of i's (i.e., only neutral agents).

3. $\Gamma$ is necessarily individually rational if and only if for each $i \in A, \Gamma(i)$ does not contain any enemies of i's. 
Proof. The proofs of these three claims are easy:

1. By definition, a coalition structure is perfect if and only if each player is in one of her favorite coalitions, which in a FEN-hedonic game means that each player is together with all her friends and no enemies.

2. For each $i \in A, i$ necessarily prefers $\{i\}$ to $\Gamma(i)$ if and only if $\Gamma(i)$ contains no friend and at least one enemy of $i$ 's.

3. For each $i \in A, i$ possibly prefers $\{i\}$ to $\Gamma(i)$ if and only if $\Gamma(i)$ contains an enemy of $i$ 's.

This completes the proof.

Example 22 Consider the FEN-hedonic game from Example 9 with three players who can be partitioned in a total of five different coalition structures. Observe that there does not exist a possibly perfect coalition structure.

While $\{\{1,2,3\}\}$ is possibly Nash stable, there does not exist a necessarily Nash stable coalition structure, as in each of the five different coalition structures, player 1 or player 2, at least possibly, wants to move to another coalition.

Coalition structure $\{\{1,2,3\}\}$ is possibly individually rational, but not necessarily individually rational due to player $2 ;\{\{1,2\},\{3\}\}$ is not possibly individually rational; the other three coalition structures are necessarily individually rational.

We furthermore consider the case of a single agent $i$ entering or leaving a coalition. We state the following characterizations, which will be useful for individual stability and contractually individual stability.

Observation 23 Consider a FEN-hedonic game $\left(A,\left(\unrhd_{1}^{+0-}, \ldots, \unrhd_{n}^{+0-}\right)\right)$.

1. For a coalition $C \subseteq A$ and two players, $j \in C$ and $i \notin C$, it holds that $C \succ_{j}^{+0-} C \cup\{i\}$ if and only if $i \in A_{j}^{-}$. Hence, $j$ possibly prefers $C$ to $C \cup\{i\}$ if and only if $j$ necessarily prefers $C$ to $C \cup\{i\}$, which in turn holds if and only if $i$ is an enemy of $j$ 's.

2. For a coalition $C \subseteq A$ and two players $i, k \in C$, it holds that $C \succ_{k}^{+0-} C \backslash\{i\}$ if and only if $i \in A_{k}^{+}$. Again, $k$ possibly prefers $C$ to $C \backslash\{i\}$ if and only if $k$ necessarily prefers $C$ to $C \backslash\{i\}$, which in turn holds if and only if $k$ considers $i$ as a friend.

Example 24 Again, consider the FEN-hedonic game from Example 9 and recall the definitions of individual stability and contractually individual stability.

For $\{\{1,3\},\{2\}\}$, it holds that player 2 possibly wants to move to $\{1,3\}$, and 1 and 2 do not see 2 as an enemy, so necessary individual stability is not satisfied. Also, since in $\{2\}$ there is no other player who considers 2 a friend, necessary contractually individual stability is not satisfied either. Observe that this coalition structure is, however, possibly individually stable and therefore also possibly contractually individually stable.

Coalition structure $\{\{1\},\{2,3\}\}$ is not possibly individually stable, as player 3 wants to join the coalition $\{1\}$ and player 1 welcomes her. Player 2, however, considers 3 a friend and 1 an enemy. Therefore, as 2 does not want to move and would not welcome 1 to join the coalition $\{2,3\}$, this coalition structure is necessarily contractually individually stable. 
To conclude this section, let us now state some easy observations about the connections among the notions of possible and necessary stability defined in Section 2.2. First, observe that if there exists a necessarily strictly popular coalition structure, it is unique, whereas there can be more than one possibly strictly popular coalition structure. Further, if there exists a necessarily strictly popular coalition structure, it is necessarily Pareto optimal. And if there exist possibly strictly popular coalition structures, each of them is possibly Pareto optimal. Finally, if there exists a unique perfect partition, it is always the unique necessarily strictly popular coalition structure.

On the other hand, a necessarily strictly popular coalition structure does not need to be possibly individually rational. Even if the possible core is nonempty, a necessarily strictly popular coalition structure does not need to be possibly core stable. And the same holds for the concepts of Nash stability, individual stability, contractual individual stability, and strict core stability.

\subsection{Possible and Necessary Stability: Problem Definitions and Overview of Complexity Results}

We are interested in properties of FEN-hedonic games and in characterizations of stability concepts in FEN-hedonic games. For some stability concepts $\gamma$, there sometimes (albeit not always) exists a coalition structure satisfying that concept (possibly or necessarily), i.e., some FEN-hedonic games have a (possibly or necessarily) $\gamma$-stable coalition structure and some have not. In these nontrivial cases, we ask how hard it is to decide whether for a given FEN-hedonic game a given coalition structure possibly or necessarily satisfies $\gamma$, and to decide whether there exists a coalition structure in a given FEN-hedonic game that possibly or necessarily satisfies $\gamma$. Similar questions are often analyzed in the context of hedonic games (Woeginger, 2013b; Aziz et al., 2013b; Rey et al., 2016). We now adapt the definition of the verification problem to the notions of possible and necessary verification, and we similarly adapt the definition of the existence problem to possible and necessary existence. Again, let $\gamma$ be one of the previously defined stability concepts for hedonic games. Possible and necessary verification for $\gamma$ are defined as follows.

\begin{tabular}{ll}
\hline Given: & \multicolumn{1}{c}{ POSSIBLE- $\gamma$-VERIFICATION } \\
Question: & $\begin{array}{l}\text { A FEN-hedonic game } H \text { and a coalition structure } \Gamma . \\
\text { Does } \Gamma \text { possibly satisfy } \gamma \text { in } H \text {, that is, does } \Gamma \text { satisfy } \gamma \text { in some profile } \\
\text { of preferences resulting from the polarized responsive extension of } H ?\end{array}$ \\
\hline & \multicolumn{1}{c}{ NECESSARY- $\gamma$-VERIFICATION } \\
\hline Given: & $\begin{array}{l}\text { A FEN-hedonic game } H \text { and a coalition structure } \Gamma . \\
\text { Question: }\end{array}$ \\
& $\begin{array}{l}\text { Does } \Gamma \text { necessarily satisfy } \gamma \text { in } H \text {, that is, does } \Gamma \text { satisfy } \gamma \text { in all profiles } \\
\text { of preferences resulting from the polarized responsive extension of } H ?\end{array}$ \\
\hline
\end{tabular}

We now define the possible existence and the necessary existence problem. In the former problem, we ask whether there is some coalition structure satisfying the stability concept $\gamma$ for some preference profile resulting from the polarized responsive extension, while in the latter problem the question is whether there is some coalition structure satisfying $\gamma$ for all preference profiles resulting from the polarized responsive extension. 


\begin{tabular}{ll}
\hline & \multicolumn{1}{c}{ POSSIBLE- $\gamma$-EXISTENCE } \\
\hline $\begin{array}{l}\text { Given: } \\
\text { Question: }\end{array}$ & $\begin{array}{l}\text { A FEN-hedonic game } H . \\
\text { Does there exist a coalition structure that possibly satisfies } \gamma \text { in } H ?\end{array}$ \\
\hline & \\
\hline & \multicolumn{1}{c}{ NECESSARY- $\gamma$-EXISTENCE } \\
\hline Given: & A FEN-hedonic game $H$. \\
\hline
\end{tabular}

Note that the above definition of NECESSARY- $\gamma$-EXISTENCE is based on one possible interpretation. Another possible interpretation would change the question of the problem to asking whether for all profiles of preferences resulting from the polarized responsive extension of the game, there exists a coalition structure satisfying the stability concept $\gamma$. Observe that the existential and the universal quantifier have been swapped in this alternative interpretation. Consequently, a yes-instance for the latter problem is also a yes-instance for the former. We will illustrate this distinction by Example 25. However, we consider the interpretation underlying NECESSARY- $\gamma$-EXISTENCE to be more natural and therefore stick to it here: With the "for all, there exists" definition we would go beyond interpreting FEN-hedonic games as concise descriptions of hedonic games, since it would amount to consider a different hedonic game for each completion. The "there exists, for all" definition, by contrast, leads to the only natural interpretation when one takes FEN-hedonic game to be a concise description of hedonic game. ${ }^{3}$

Example 25 Consider the following game with three players, $A=\{1,2,3\}$, and with $\unrhd_{1}^{+0-}=$ $(2|\{3\}| \emptyset), \unrhd_{2}^{+0-}=(1|\{3\}| \emptyset)$, and $\unrhd_{3}^{+0-}=(1|\emptyset| 2)$. We obtain the following polarized responsive orders: $\{1,2\} \sim_{1}\{1,2,3\} \succ_{1}\{1\} \sim_{1}\{1,3\},\{1,2\} \sim_{2}\{1,2,3\} \succ_{2}\{2\} \sim_{2}\{2,3\}$, and $\{1,3\} \succ_{3}\{3\} \succ_{3}\{2,3\}$ and $\{1,3\} \succ_{3}\{1,2,3\} \succ_{3}\{2,3\}$, while $\{3\}$ and $\{1,2,3\}$ are incomparable for 3. Any coalition structure in which players 1 and 2 are not in the same coalition cannot possibly be Nash stable. On the one hand, $\{\{1,2\},\{3\}\}$ is Nash stable if and only if a preference extension provides $\{3\} \succeq_{3}\{1,2,3\}$. On the other hand, $\{\{1,2,3\}\}$ is Nash stable if and only if $\{1,2,3\} \succeq_{3}\{3\}$ in a preference extension. Thus, for every preference extension, there certainly exists a Nash stable coalition structure. However, there is no necessarily Nash stable coalition structure.

Table 3 sums up the computational complexity results for possible and necessary stability verification and existence. In what follows, we will provide our complexity results for the various stability concepts from Section 2.2.

\subsection{Perfectness and Individual Rationality}

We now give a simple, polynomial characterization of necessary (and, equivalently, possibly) perfectness in FEN-hedonic games. Given a FEN-hedonic game $G=\left(A,\left(\unrhd_{1}^{+0-}, \ldots, \unrhd_{n}^{+0-}\right)\right)$, the friendship graph $F_{G}=(A, E)$ is the undirected graph whose set of vertices is $A$ and that contains edge $\{i, j\}$ if and only if $i \in A_{j}^{+}$or $j \in A_{i}^{+}$. We write $F_{G}(i)=\{i\} \cup\{j \mid\{i, j\} \in E\}$. Let $F_{G}^{*}$ be the transitive closure of $F_{G}$.

\footnotetext{
${ }^{3}$ We thank an anonymous reviewer for this useful remark.
} 


\begin{tabular}{|c|c|c|c|c|}
\hline \multirow[b]{2}{*}{$\gamma$} & \multicolumn{2}{|c|}{ VERIFICATION } & \multicolumn{2}{|c|}{ EXISTENCE } \\
\hline & Possible & NECESSARY & Possible & NECESSARY \\
\hline perfectness & in $\mathrm{P}$ (Cor. 28$)$ & in $\mathrm{P}$ (Cor. 28$)$ & in $\mathrm{P}$ (Cor. 28$)$ & in $\mathrm{P}($ Cor. 28$)$ \\
\hline ind. rationality & in P (Prop. 29) & in P (Prop. 29) & in P (Prop. 29) & in P (Prop. 29) \\
\hline Nash stability & in P (Thm. 39) & in P (Thm. 36) & $\begin{array}{l}\text { NP-complete } \\
\text { (Thm. 43) }\end{array}$ & $\begin{array}{l}\text { NP-complete } \\
\text { (Thm. 44) }\end{array}$ \\
\hline ind. stability & in $\mathrm{P}$ (Thm. 40) & in $\mathrm{P}$ (Thm. 37) & in NP & $\begin{array}{l}\text { NP-complete } \\
\text { (Thm. } 48 \text { ) }\end{array}$ \\
\hline contr. ind. stability & in $\mathrm{P}(\mathrm{Thm} .41)$ & in $\mathrm{P}$ (Thm. 38) & in $\mathrm{P}$ (Thm. 45) & in NP \\
\hline core stability & $\begin{array}{l}\text { coNP-complete } \\
\text { (Thm. 54) }\end{array}$ & $\begin{array}{l}\text { in coNP } \\
\text { (Cor. 53) }\end{array}$ & in $\Sigma_{2}^{p}$ & $\operatorname{in} \Sigma_{2}^{p}$ \\
\hline str. core stability & $\begin{array}{l}\text { coNP-complete } \\
\text { (Thm. 54) }\end{array}$ & $\begin{array}{l}\text { in coNP } \\
\text { (Cor. 53) }\end{array}$ & in $\Sigma_{2}^{p}$ & in $\Sigma_{2}^{p}$ \\
\hline Pareto optimality & $\begin{array}{l}\text { coNP-complete } \\
\text { (Thm. 35) }\end{array}$ & $\begin{array}{l}\text { coNP-complete } \\
\text { (Thm. 35) }\end{array}$ & in P (Thm. 30) & in $\Sigma_{2}^{p}$ \\
\hline popularity & $\begin{array}{l}\text { coNP-complete } \\
\text { (Thm. 61) }\end{array}$ & $\begin{array}{l}\text { coNP-complete } \\
\text { (Thm. 61) }\end{array}$ & in $\Sigma_{2}^{p}$ & in $\Sigma_{2}^{p}$ \\
\hline str. popularity & $\begin{array}{l}\text { coNP-complete } \\
\text { (Thm. 61) }\end{array}$ & $\begin{array}{l}\text { coNP-complete } \\
\text { (Thm. 61) }\end{array}$ & $\begin{array}{l}\text { coNP-hard, } \\
\text { in } \Sigma_{2}^{p}(\text { Thm. 62) }\end{array}$ & $\begin{array}{l}\text { coNP-hard, } \\
\text { in } \Sigma_{2}^{p}(\mathrm{Thm} .62)\end{array}$ \\
\hline
\end{tabular}

Table 3: Overview of complexity results on verification and existence problems for possible and necessary stability notions in FEN-hedonic games

Proposition 26 There is a necessarily perfect partition (and, equivalently, a possibly perfect partition) for $G$ if for each $i, j$ such that $i \in A_{j}^{-}, F_{G}$ contains no path from $i$ to $j$, and in that case a necessarily perfect partition for $G$ can be computed by Algorithm 1.

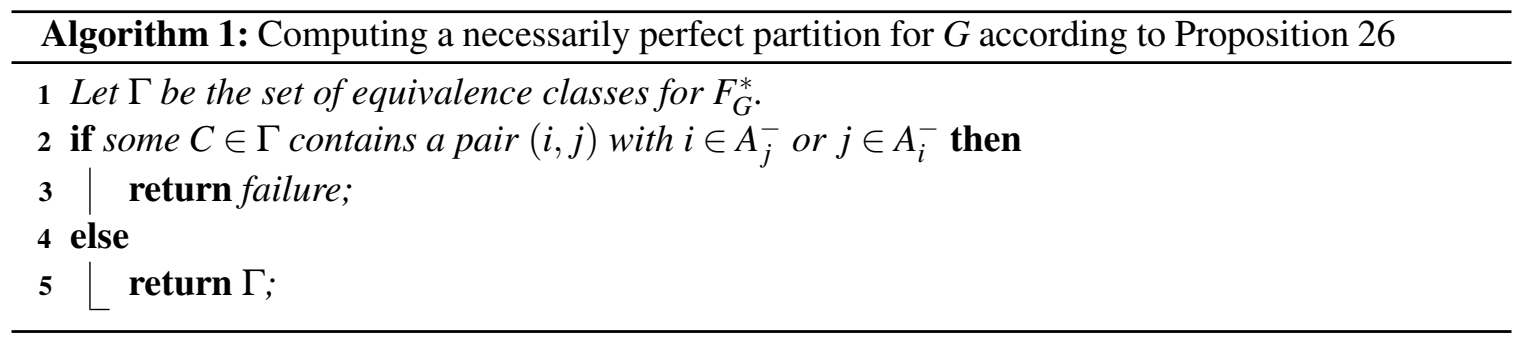

Proof. If Algorithm 1 returns $\Gamma$, then we check that each agent is in (one of) her best possible coalition(s): For any $i$, because of the construction of the coalition $\Gamma(i)$ containing $i$, all friends of $i$ must be in $\Gamma(i)$, and because the failure condition is not satisfied, no enemy of $i$ is in $\Gamma(i)$. Therefore, $\Gamma$ is necessarily perfect. (Note that there can be more than one perfect partition due to disconnected neutral agents.)

If there are two agents, say $i$ and $j$, such that $j \in F_{G}^{*}(i)$ but $j \notin \Gamma(i)$, then by definition of $F_{G}^{*}(i)$ there must be two agents, $k$ and $k^{\prime}$, such that $k \in F_{G}^{*}(i), k^{\prime} \notin F_{G}^{*}(i)$, and $F_{G}$ contains $\left(k, k^{\prime}\right)$. Then either $k$ or $k^{\prime}$ is not in her best possible coalition, and $\Gamma$ is not necessarily perfect. Therefore, if $\Gamma$ 
is a necessarily perfect partition, then (1) for each $i$ we have $\Gamma(i) \supseteq F_{G}^{*}(i)$. Now, if the algorithm returns failure, then for any $\Gamma$ satisfying (1) there must be an agent who is in the same coalition as one of her enemies. Therefore, there exists no necessarily perfect partition.

Example 27 Let $A=\{1,2,3,4,5,6,7\}, A_{1}^{+}=\{2\}, A_{2}^{+}=\emptyset, A_{3}^{+}=\{1,4\}, A_{4}^{+}=\{2\}, A_{5}^{+}=\emptyset, A_{6}^{+}=$ $\{5\}, A_{7}^{+}=\emptyset, A_{1}^{-}=\{6\}, A_{2}^{-}=\{5\}, A_{3}^{-}=A_{4}^{-}=A_{5}^{-}=A_{7}^{-}=\emptyset, A_{6}^{-}=\{1,7\}$. We start by constructing $F_{G}^{*}(k)$, which contains the equivalence classes $\{1,2,3,4\},\{5,6\}$, and $\{7\}$. None of them contains two agents, $i$ and $j$, with $i$ being an enemy of $j$; therefore, $\Gamma=\{\{1,2,3,4\},\{5,6\},\{7\}\}$ is necessarily perfect. Note that $\Gamma=\{\{1,2,3,4,7\},\{5,6\}\}$ is also necessarily perfect (and there is no other necessarily perfect partition). If, on the other hand, we change $A_{2}^{+}$into $A_{2}^{+}=\left\{A_{5}\right\}$, then $F_{G}^{*}(k)$ contains the equivalence classes $\{1,2,3,4,5,6\},\{7\}$, and because 1 is an enemy of 6 , there is no necessarily perfect partition.

Corollary 28 All variants of verification and existence problems regarding perfectness are in $\mathrm{P}$.

The same is true for all possible and necessary verification and existence problems with respect to individual rationality.

Proposition 29 All variants of verification and existence problems regarding individual rationality are in $\mathrm{P}$.

Proof. Obviously, there always is a necessarily individually rational coalition structure (namely, the coalition structure where every agent is on her own). By the characterization in Proposition 21, possible and necessary individual rationality can be verified easily.

\subsection{Pareto Optimality}

We now give some results for possible and necessary Pareto optimality and start with the existence problem for possible Pareto optimality.

Theorem 30 There always exists a possibly Pareto optimal coalition structure in a FEN-hedonic game.

Proof. We apply serial dictatorship. Let $\mathscr{C}_{1}$ be the set of all coalition structures most preferred by player 1 (which are all coalition structures in which 1's coalition contains all her friends and none of her enemies); and for each $j=2, \ldots, n$, let $\mathscr{C}_{j}$ be the set of all coalition structures $\Gamma$ in $\mathscr{C}_{j-1}$ such that there exists no $\Gamma^{\prime} \in \mathscr{C}_{j-1}$ with $\Gamma^{\prime} \succ_{j}^{+0-} \Gamma$. Let $\Gamma^{*}$ be an arbitrary coalition structure in $\mathscr{C}_{n}$. We claim that $\Gamma^{*}$ is possibly Pareto optimal. Assume not: Let $\Gamma^{\prime}$ such that $\Gamma^{\prime}(i) \succeq_{i}^{+0-} \Gamma^{*}(i)$ for all $i$, and $\Gamma^{\prime}(j) \succ_{j}^{+0-} \Gamma^{*}(j)$ for some $j$. Let $j^{*}$ be the smallest $j$ with $\Gamma^{\prime}(j) \succ_{j}^{+0-} \Gamma^{*}(j)$. Observe that $\Gamma^{\prime} \in \mathscr{C}_{j^{*}-1}$. (If this wasn't the case then $\Gamma^{*}$ wouldn't be in $\mathscr{C}_{j^{*}-1}$ either, because every player weakly prefers $\Gamma^{\prime}$ to $\Gamma^{*}$.) Then $\Gamma^{*}$ should not have been included in $\mathscr{C}_{j^{*}}$ since $\Gamma^{\prime} \in \mathscr{C}_{j^{*}-1}$ and $\Gamma^{\prime}\left(j^{*}\right) \succ_{j^{*}}^{+0-} \Gamma^{*}\left(j^{*}\right):$ a contradiction.

Consequently, Possible-Pareto-Optimality-Existence is in P. However, the same does not hold for NECESSARY-PARETO-OPTIMALITY-EXISTENCE since there exist both yes- and noinstances for this problem. The following example shows a FEN-hedonic games for which there is no necessarily Pareto optimal coalition structure. 
Example 31 Let $H=\left(A, \unrhd^{+0-}\right)$ be a FEN-hedonic game with $A=\{1,2,3, f\}$ and let $\unrhd^{+0-}$ consist of the following weak rankings with double threshold: $\unrhd_{1}^{+0-}=(f|3| 2), \unrhd_{2}^{+0-}=(f|1| 3)$, $\unrhd_{3}^{+0-}=(f|2| 1)$, and $\unrhd_{f}^{+0-}=(\emptyset|1,2,3| \emptyset)$.

It then holds that there doesn't exist any necessarily Pareto optimal coalition structure, i.e., for every coalition structure $\Gamma$ there is an extended profile $\succeq=\left(\succeq_{1}, \ldots, \succeq_{n}\right)$ and a coalition structure $\Delta \neq \Gamma$ such that $\Delta$ Pareto-dominates $\Gamma$ in $(A, \succeq)$.

First, every coalition structure, where some of 1, 2, and 3 are in the same coalition, is possibly dominated by $\Delta=\{\{1\},\{2\},\{3\},\{f\}\}$. For example, if $\Gamma_{1}=\{\{1,2, f\},\{3\}\}$, then we consider an extended profile with $\{1\} \succ_{1}\{1,2, f\}$ and $\{2\} \succ_{2}\{1,2, f\}$. These extensions exist since $\{1\}$ and $\{1,2, f\}$ are incomparable with respect to $\succeq_{1}^{+0-}$, and so they are for $\succeq_{2}^{+0-}$. However, $\Delta$ is also possibly dominated by $\Gamma_{1}$. To see this, we just consider an extended profile with $\{1,2, f\} \succ_{1}\{1\}$ and $\{1,2, f\} \succ_{2}\{2\}$. Finally, all remaining coalition structures have the form $\Gamma=\{\{i, f\},\{j\},\{k\}\}$ with $\{i, j, k\}=\{1,2,3\}$ and are possibly dominated by $\{\{j, i, f\},\{k\}\}$ where $j$ is the player who sees $i$ as an enemy.

Now we turn to the verification variants.

Proposition $32 \Gamma$ is not possibly Pareto optimal if and only if there exists a coalition structure $\Delta$ such that (1) for all $i \in A$ we have $\Delta(i) \succeq_{i}^{+\Gamma(i)} \Gamma(i)$, and (2) for some $i \in A$ we have $\Delta(i) \succ_{i}^{+\Gamma(i)} \Gamma(i)$.

Proof. From right to left, assume there exists a coalition structure $\Delta$ such that (1) and (2) hold. Then, for any $\succeq=\left(\succeq_{1}, \ldots, \succeq_{n}\right) \in \times_{i=1}^{n} \operatorname{Ext}\left(\succeq_{i}^{+0-}\right)$, by Propositions 19.2(c) and 19.2(d) we have $\Delta(i) \succeq_{i} \Gamma(i)$ for all $i \in A$, and $\Delta(i) \succ_{i} \Gamma(i)$ for some $i \in A$, which means that $\Delta$ Pareto-dominates $\Gamma$ with respect to $\succeq$. Therefore, $\Gamma$ is not possibly Pareto optimal.

From left to right, assume that $\Gamma$ is not possibly Pareto optimal. Then, for any profile $\succeq \epsilon$ $\times_{i=1}^{n} \operatorname{Ext}\left(\succeq_{i}^{+0-}\right)$, there exists a coalition structure $\Delta$ that Pareto-dominates $\Gamma$ with respect to $\succeq$. Consider a profile $\left(\succeq_{1}, \ldots, \succeq_{n}\right) \in \times_{i=1}^{n} \operatorname{Ext}\left(\succeq_{i}^{+\Gamma(i)}\right)$. Such a profile exists because $\succ_{i}^{+\Gamma(i)}$ is acyclic due to Proposition 16. It then holds for every $i \in A$ that $\succeq_{i}$ also extends $\succeq_{i}^{+0-}$. Hence, there also is a coalition structure $\Delta$ for this profile that Pareto-dominates $\Gamma$, i.e., such that $\Delta(i) \succeq_{i} \Gamma(i)$ for all $i \in A$, and $\Delta(i) \succ_{i} \Gamma(i)$ for some $i \in A$. Since $\succeq_{i}$ extends $\succeq_{i}^{+\Gamma(i)}$ and with Observation 18 it follows that $\Delta(i) \succeq_{i}^{+\Gamma(i)} \Gamma(i)$ for every $i \in A$, and $\Delta(i) \succ_{i}^{+\Gamma(i)} \Gamma(i)$ for some $i \in A$.

Similarly, we get the following result.

Proposition $33 \Gamma$ is not necessarily Pareto optimal if and only if there exists a coalition structure $\Delta$ such that (1) for all $i \in A$ we have $\Delta(i) \succeq_{i}^{-\Gamma(i)} \Gamma(i)$, and (2) for some $i \in A$ we have $\Delta(i) \succ_{i}^{-\Gamma(i)} \Gamma(i)$.

Proof. From right to left, assume there exists a coalition structure $\Delta$ such that (1) and (2) hold. Then, by Propositions 19.1(c) and 19.1(d), there is a $\succeq=\left(\succeq_{1}, \ldots, \succeq_{n}\right) \in \times_{i=1}^{n} \operatorname{Ext}\left(\succeq_{i}^{+0-}\right)$ such that we have $\Delta(i) \succeq_{i} \Gamma(i)$ for all $i \in A$, and $\Delta(i) \succ_{i} \Gamma(i)$ for some $i \in A$, which means that $\Delta$ Paretodominates $\Gamma$ with respect to $\succeq$. Therefore, $\Gamma$ is not necessarily Pareto optimal.

From left to right, assume that $\Gamma$ is not necessarily Pareto optimal. Then there exists a profile $\left(\succeq_{1}, \ldots, \succeq_{n}\right) \in \times_{i=1}^{n} \operatorname{Ext}\left(\succeq_{i}^{+0-}\right)$ and a coalition structure $\Delta$ such that $\Delta(i) \succeq_{i} \Gamma(i)$ for all $i \in A$, and $\Delta(i) \succ_{i} \Gamma(i)$ for some $i \in A$. By Propositions 19.1(c) and 19.1(d), this means that $\Delta(i) \succeq_{i}^{-\Gamma(i)} \Gamma(i)$ for all $i \in A$, and $\Delta(i) \succ_{i}^{-\Gamma(i)} \Gamma(i)$ for some $i \in A$. 
By Propositions 32 and 33, we get coNP upper bounds for the two verification variants of Pareto optimality.

Corollary 34 Possible-PAReto-Optimality-VerificAtion and Necessary-PAReto-OpTIMALITY-VERIFICATION are in coNP.

Theorem 35 Possible-PARETO-Optimality-VERIFICATION and NeCESSARY-PARETO-OPTIMALITY-VERIFICATION are coNP-complete.

Proof. To show coNP-hardness of the first problem, we provide a reduction from the NP-complete problem X3C (which was defined in Section 2.3) to the complement of Possible-PARETO-OPTIMALITY-VERIFICATION. Letting $(B, \mathscr{S})$ with $|B|=3 \mathrm{~m}$ be a given X3C instance, we construct the following game. The set of players consists of two types of players which we call element and set players: $A=\left\{\beta_{b} \mid b \in B\right\} \cup \bigcup_{S \in \mathscr{S}} Q_{S}$. In the following, we describe them with their weak rankings with double threshold. Recall from Section 3.1 that for a set $X$ of players, $X_{\sim_{i}}$ denotes that player $i$ is indifferent between any pair of players $x, y \in X$. For the sake of readability, we will here and in some later proofs omit the subscript specifying the player $i$ and simply write $X_{\sim}$ for $X_{\sim_{i}}$ when $i$ is clear from the context. (Since players always are indifferent with respect to their neutral players, we even omit the subscript $\sim$ and simply write $X$ when $X$ are the neutral players.) We will also drop these subscripts within the weak rankings and write $\triangleright$ instead of $\triangleright_{i}$ when $i$ is clear from the context. The set " $\{$ other players\}" in these weak rankings refers to all players that are not mentioned.

Element players: For each $b \in B$, there is one element player $\beta_{b}$ who has all set players (to be defined below) corresponding to sets $S$ containing $b$ as her highest ranked friends, followed by the remaining element players. There are no neutral players, so all other players are in her set of enemies. Formally, for each $b \in B$,

$$
\unrhd_{\beta_{b}}^{+0-}=\left(\bigcup_{\{S \mid b \in S\}} Q_{S \sim} \triangleright\left\{\beta_{b^{\prime}} \mid b^{\prime} \neq b\right\}_{\sim}|\emptyset|\{\text { other players }\}_{\sim}\right) .
$$

Set players: For each $S \in \mathscr{S}$, we have $3 m-3$ set players in the set $Q_{S}=\left\{\zeta_{S, k} \mid 1 \leq k \leq 3 m-3\right\}$. For each fixed $S \in \mathscr{S}$, these players only consider the "next" set player in $Q_{S}$ to be her friend (except for the last player in each $Q_{S}$ ). The remaining set players in their $Q_{S}$-set and the element players corresponding to the elements in the respective $S$ are neutral players while all other players are their enemies. Formally, for each $S \in \mathscr{S}$ and for each $k, 1 \leq k \leq 3 m-4$,

$$
\begin{aligned}
\unrhd_{\zeta_{S, k}}^{+0-} & \left.=\left(\left\{\zeta_{S, k+1} \mid k^{\prime} \neq k\right\}_{\sim}\left|\left\{\zeta_{S, k^{\prime}} \mid k \neq k^{\prime} \neq k+1\right\} \cup\left\{\beta_{b} \mid b \in S\right\}\right| \text { \{other players }\right\}_{\sim}\right), \\
\unrhd_{\zeta_{S, 3 m-3}}^{+0-} & \left.=\left(\emptyset\left|\left\{\zeta_{S, k^{\prime}} \mid k^{\prime} \neq k\right\} \cup\left\{\beta_{b} \mid b \in S\right\}\right| \text { \{other players }\right\}_{\sim}\right) .
\end{aligned}
$$

This profile can be determined in polynomial time and is visualized in Figure 5.

For each $S \in \mathscr{S}$, we define $P_{S}=Q_{S} \cup\left\{\beta_{b} \mid b \in S\right\}$ and fix $\Gamma=\left\{\left\{\beta_{b} \mid b \in B\right\}\right\} \cup\left\{Q_{S} \mid S \in \mathscr{S}^{\prime}\right\}$ to be our coalition structure. It holds that $(B, \mathscr{S})$ belongs to $\mathrm{X} 3 \mathrm{C}$ if and only if $\Gamma$ is not possibly Pareto optimal.

Only if: Consider a solution $\mathscr{S}^{\prime}$ for $(B, \mathscr{S})$, assuming there is one. The coalition structure $\Gamma^{\prime}=\left\{P_{S} \mid S \in \mathscr{S}^{\prime}\right\} \cup\left\{Q_{S} \mid S \notin \mathscr{S}^{\prime}\right\}$ necessarily Pareto-dominates $\Gamma$ : Each player $\zeta_{S, k}, S \in \mathscr{S}$, $1 \leq k \leq 3 m-3$, is indifferent between $Q_{S}$ and $P_{S}$, as $\beta_{b}, b \in S$, is considered as neutral. Furthermore, 


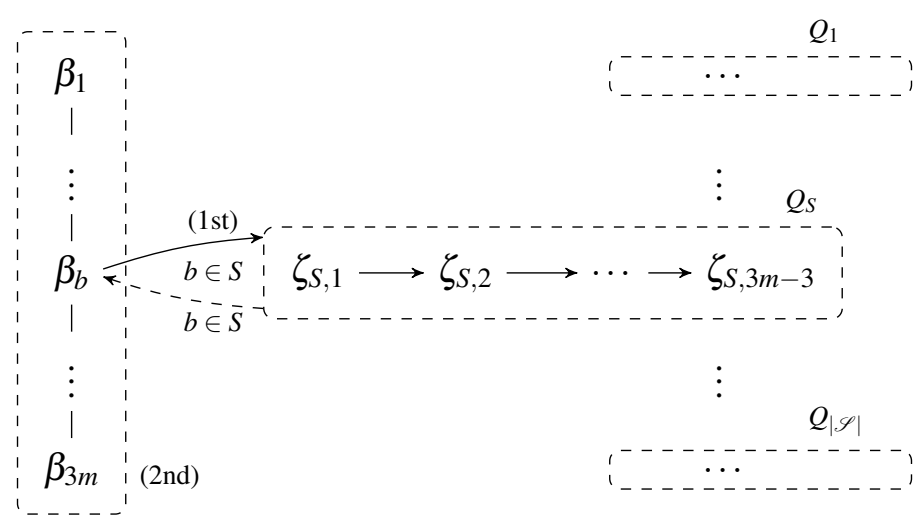

Figure 5: Network of friends for the construction in the proof of Theorem 35

each $\beta_{b}, b \in B$, necessarily strictly prefers $P_{S}$ to $\Gamma\left(\beta_{b}\right)$, since two friends can be mapped to two indifferent friends, and $3 m-3$ players can be mapped to higher ranked players, and $\beta_{b}$ has got no enemies in either coalition.

If: Assume there exists a coalition structure $\Gamma^{\prime}$ that necessarily Pareto-dominates $\Gamma$, that is, $\Gamma^{\prime}(i) \succeq_{i} \Gamma(i)$ for each player $i$, and $\Gamma^{\prime}(j) \succ_{j} \Gamma(j)$ for at least one player $j$.

From the point of view of players $\zeta_{S, k}, S \in \mathscr{S}, 1 \leq k \leq 3 m-3$, the players in $Q_{S}$ have to be together in one coalition in $\Gamma^{\prime}$ and without any enemies. A player $\beta_{b}$ necessarily prefers $P_{S}$ to $\left\{\beta_{b^{\prime}} \mid b^{\prime} \in B\right\}$ and the latter possibly to every other coalition containing $\beta_{b}$. Since $\Gamma^{\prime}$ necessarily Pareto-dominates $\Gamma$, there is a preference extension for which the only possible $\Gamma^{\prime}$ assigns each $\beta_{b}$, $b \in B$, to a $P_{S}$, which implies that there is an exact cover of $B$ in $\mathscr{S}$.

For the second problem, NeCESSARY-PAREto-Optimality-Verification, we can show coNP-hardness by slightly changing the construction: Now there are only $3 m-2$ players $\zeta_{S, k}$ for each $S \in \mathscr{S}$ and each $\beta_{b}, b \in B$, prefers each $\beta_{b^{\prime}}, b \neq b^{\prime}$ to $\zeta_{S, k}, r \in S, 1 \leq k \leq 3 m-2$. Observe that with an analogous argumentation, changing the relations of possible and necessary preferences, $(B, \mathscr{S})$ is a positive instance of $\mathrm{X} 3 \mathrm{C}$ if and only if $\Gamma$ is not necessarily Pareto optimal.

The proof is complete, since Possible-Pareto-Optimality-Verification and NecesSARY-PARETO-OPTIMALITY-VERIFICATION are in coNP by Corollary 34.

\subsection{Verification for Nash Stability, Individual Stability, and Contractually Individual Stability}

We now turn to the verification problems for Nash, individual, and contractually individual stability. We show that both possible and necessary verification are easy for these three concepts. 


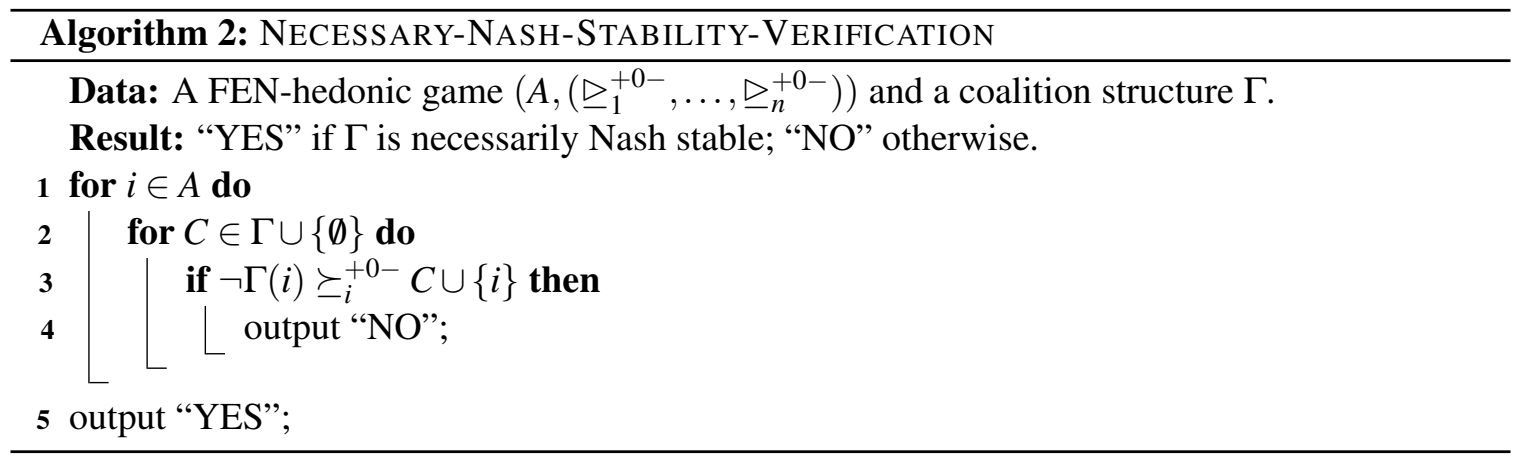

\section{Theorem 36 NeCESSARY-NASH-STABILITY-VERIFICATION is in P.}

Proof. Given a FEN-hedonic game $\left(A, \unrhd^{+0-}\right)$ and a coalition structure $\Gamma$, it is possible to determine whether $\Gamma$ is necessarily Nash stable in polynomial time. This can be done by Algorithm 2 .

For a given FEN-hedonic game $\left(A,\left(\unrhd_{1}^{+0-}, \ldots, \unrhd_{n}^{+0-}\right)\right)$, a coalition structure $\Gamma=\left\{C_{1}, \ldots, C_{k}\right\}$, $1 \leq k \leq n$, is necessarily Nash stable if for all profiles $\left(\succeq_{1}, \ldots, \succeq_{n}\right) \in \times_{i=1}^{n} \operatorname{Ext}\left(\succeq_{i}^{+0-}\right)$, all agents $i \in A$, and all coalitions $C \in \Gamma \cup\{\emptyset\}$ it holds that $\Gamma(i) \succeq_{i} C \cup\{i\}$. Therefore, we just need to check if we can extend $\succeq^{+0-}$ in such a way that $\Gamma(i) \succeq_{i} C \cup\{i\}$ does not hold for some $i \in A$ and $C \in \Gamma \cup\{\emptyset\}$. If this is the case, then $\Gamma$ is not necessarily Nash stable.

Hence, we iterate all $i \in A$ and $C \in \Gamma \cup\{\emptyset\}$. There are four cases possible: (1) $\Gamma(i) \succ_{i}^{+0-} C \cup\{i\}$, (2) $\Gamma(i) \sim_{i}^{+0-} C \cup\{i\}$, (3) $\Gamma(i) \prec_{i}^{+0-} C \cup\{i\}$, or (4) $\Gamma(i)$ and $C \cup\{i\}$ are incomparable. In Cases (1) and (2), Nash stability is clearly not violated. Therefore, the algorithm just continues with the next iteration. If $\Gamma(i) \prec_{i}^{+0-} C \cup\{i\}$, this is clearly violating Nash stability and "NO" is output. If $\Gamma(i)$ and $C \cup\{i\}$ are incomparable, then it is possible to set $\Gamma(i) \prec_{i} C \cup\{i\}$ in the extension $\succeq_{i}$ of $\succeq_{i}^{+0-}$ such that Nash stability is violated. Accordingly, "NO" is output in this case. If "NO" is not output at any moment, then $\Gamma(i) \succeq_{i}^{+0-} C \cup\{i\}$ holds for all $i \in A$ and $C \in \Gamma \cup\{\emptyset\}$. Hence, "YES" is output.

The outer for-loop (line 1) runs exactly $|A|=n$ times. The inner for-loop (line 2) runs $|\Gamma \cup\{\emptyset\}|=$ $k+1 \leq n+1$ times. The relation between $\Gamma(i)$ and $C \cup\{i\}$ (line 3) can be checked in polynomial time by Proposition 6. Therefore, the whole algorithm runs in polynomial time.

Note that Algorithm 2 basically checks whether $\Gamma(i) \succeq_{i}^{-\Gamma(i)} C \cup\{i\}$ holds for all $i \in A$ and $C \in \Gamma \cup\{\emptyset\}$, which is equivalent to $\Gamma$ being necessarily Nash stable.

\section{Theorem 37 Necessary-INDiVIDUAL-STABILITY-VERIFICATION is in P.}

Proof. Algorithm 3 solves NeCESSARY-INDIVIDUAL-STABILITY-VERIFICATION in polynomial time.

For a given FEN-hedonic game $\left(A,\left(\unrhd_{1}^{+0-}, \ldots, \unrhd_{n}^{+0-}\right)\right)$, a coalition structure $\Gamma$ is necessarily individually stable if it holds for all profiles $\left(\succeq_{1}, \ldots, \succeq_{n}\right) \in \times_{i=1}^{n} \operatorname{Ext}\left(\succeq_{i}^{+0-}\right)$, all agents $i \in A$, and all coalitions $C \in \Gamma \cup\{\emptyset\}$ that $\Gamma(i) \succeq_{i} C \cup\{i\}$ or that there is an agent $j \in C$ such that $C \succ_{j} C \cup\{i\}$. By Observation 23, the last term is equivalent to $i \in A_{j}^{-}$.

Therefore, we just need to check if we can extend $\succeq^{+0-}$ in such a way that this condition does not hold: If this is the case, then $\Gamma$ is not necessarily individually stable. Hence, we iterate all $i \in A$ and $C \in \Gamma \cup\{\emptyset\}$. First, we check whether or not $\Gamma(i) \succeq_{i}^{+0-} C \cup\{i\}$ is true for the current $i$ and $C$. If so, this $i$ and $C$ do not contradict individual stability in any extension of $\succeq_{i}^{+0-}$; and if this is not 


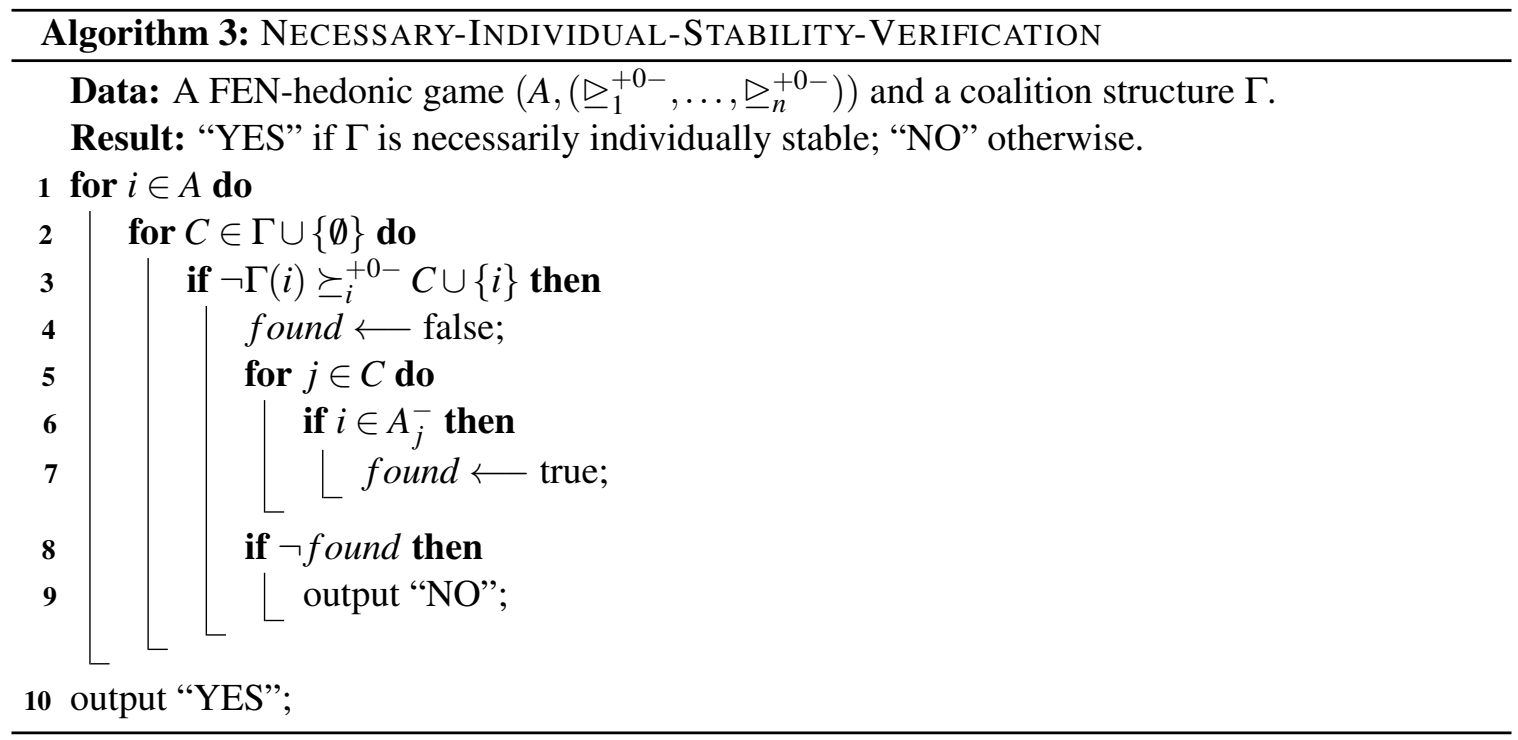

the case, there is an extension $\succeq_{i}$ of $\succeq_{i}^{+0-}$ such that $\Gamma(i) \prec_{i} C \cup\{i\}$. Then, in order for this $i$ and $C$ to not contradict individual stability, there has to be an agent $j \in C$ with $i \in A_{j}^{-}$. Therefore, we check this condition in lines 5 to 9 . If such a $j \in C$ is found, $i$ and $C$ do not contradict individual stability in any extension, and we proceed to the next iteration; otherwise, we output "NO" because we have found $i$ and $C$ witnessing that individual stability does not hold in some extension. If "NO" was not output during any iteration, then the condition stated above has to be true for every profile $\left(\succeq_{1}, \ldots, \succeq_{n}\right) \in \times_{i=1}^{n} \operatorname{Ext}\left(\succeq_{i}^{+0-}\right)$ and "YES" is output because $\Gamma$ is necessarily individually stable.

The outer for-loop (line 1) runs exactly $|A|=n$ times. The second for-loop (line 2) runs $\mid \Gamma \cup$ $\{\emptyset\} \mid \leq n+1$ times. The inner for-loop (line 5) runs $|C| \leq n$ times. The relation in line 3 can be checked in polynomial time by Proposition 6 . Therefore, the whole algorithm runs in polynomial time.

The same can be shown for the verification problem regarding necessary contractually individual stability.

\section{Theorem 38 NECESSARY-CONTRACTUALLY-INDIVIDUAL-STABILITY-VERIFICATION is in P.}

Proof. Algorithm 4 solves Necessary-Contractually-Individual-STABILITY-VerifiCATION in polynomial time.

For a given FEN-hedonic game $\left(A,\left(\unrhd_{1}^{+0-}, \ldots, \unrhd_{n}^{+0-}\right)\right)$, a coalition structure $\Gamma$ is necessarily contractually individually stable if for all profiles $\left(\succeq_{1}, \ldots, \succeq_{n}\right) \in \times_{i=1}^{n} \operatorname{Ext}\left(\succeq_{i}^{+0-}\right)$, all agents $i \in A$, and all coalitions $C \in \Gamma \cup\{\emptyset\}$ it holds that $\Gamma(i) \succeq_{i} C \cup\{i\}$ or there is an agent $j \in C$ with $C \succ_{j} C \cup\{i\}$ or there is an agent $k \in \Gamma(i)$ with $\Gamma(i) \succ_{k} \Gamma(i) \backslash\{i\}$. By Observation 23, $C \succ_{j} C \cup\{i\}$ is equivalent to $i \in A_{j}^{-}$and $\Gamma(i) \succ_{k} \Gamma(i) \backslash\{i\}$ is equivalent to $i \in A_{k}^{+}$.

Thus, Algorithm 4 checks whether for all profiles $\left(\succeq_{1}, \ldots, \succeq_{n}\right) \in \times_{i=1}^{n} \operatorname{Ext}\left(\succeq_{i}^{+0-}\right)$ and for every agent $i \in A$, one of the following two conditions holds: (a) there exists an agent $k \in \Gamma(i)$ who sees $i$ as a friend; (b) for all coalitions $C \in \Gamma \cup\{\emptyset\}$, it holds that $\Gamma(i) \succeq_{i} C \cup\{i\}$ or there exists an agent $j \in C$ who sees $i$ as an enemy. Analogously to the proof of Theorem 37, it is easy to see that Algorithm 4 runs in polynomial time. 


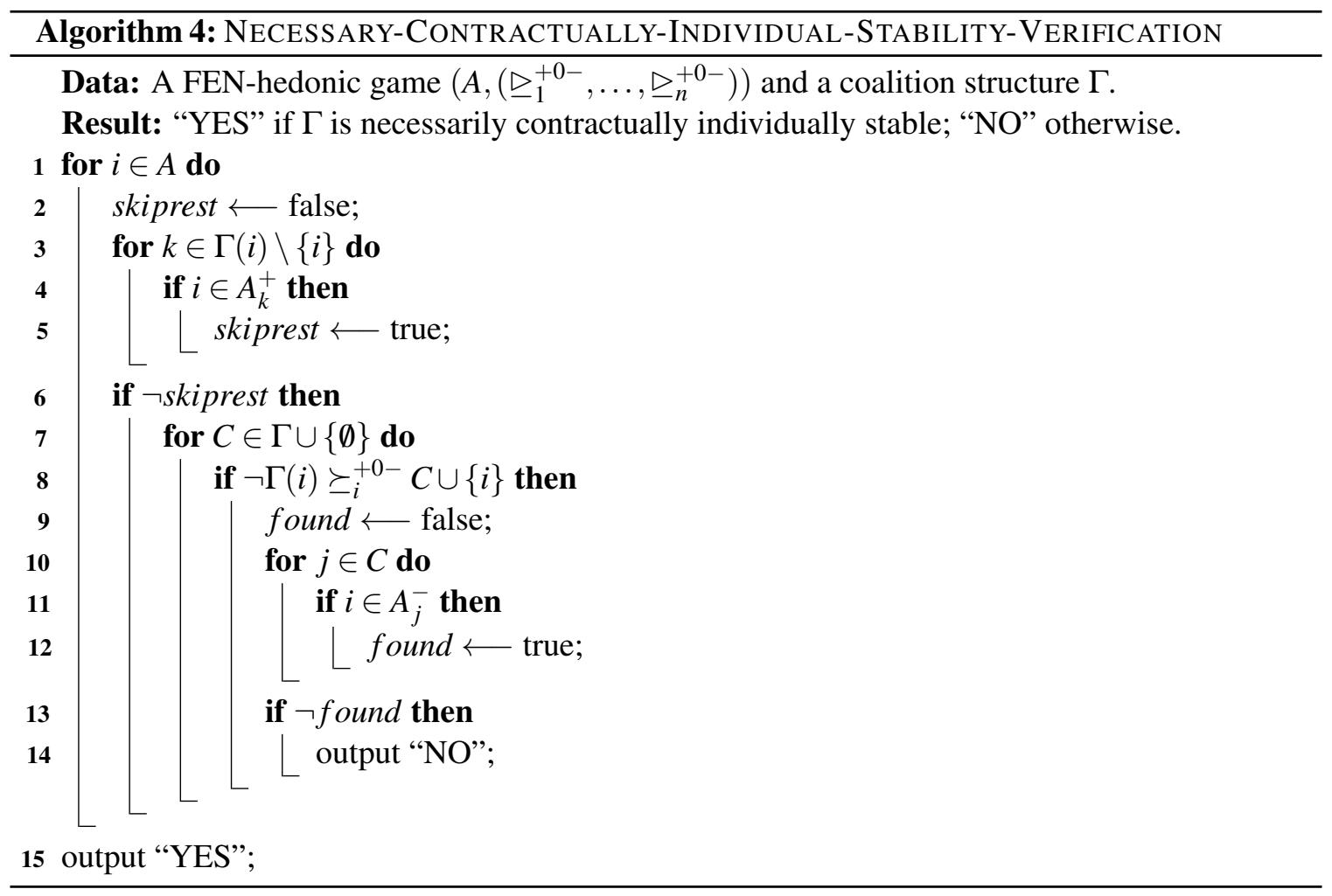

We now turn to the verification problem for possible Nash, individual, and contractually individual stability, again showing that these problems can be solved efficiently. We start with possible Nash stability.

\section{Theorem 39 Possible-Nash-Stability-Verification is in $\mathrm{P}$.}

Proof. The following algorithm solves Possible-NASH-STABILITY-VERIFICATION in polynomial time.

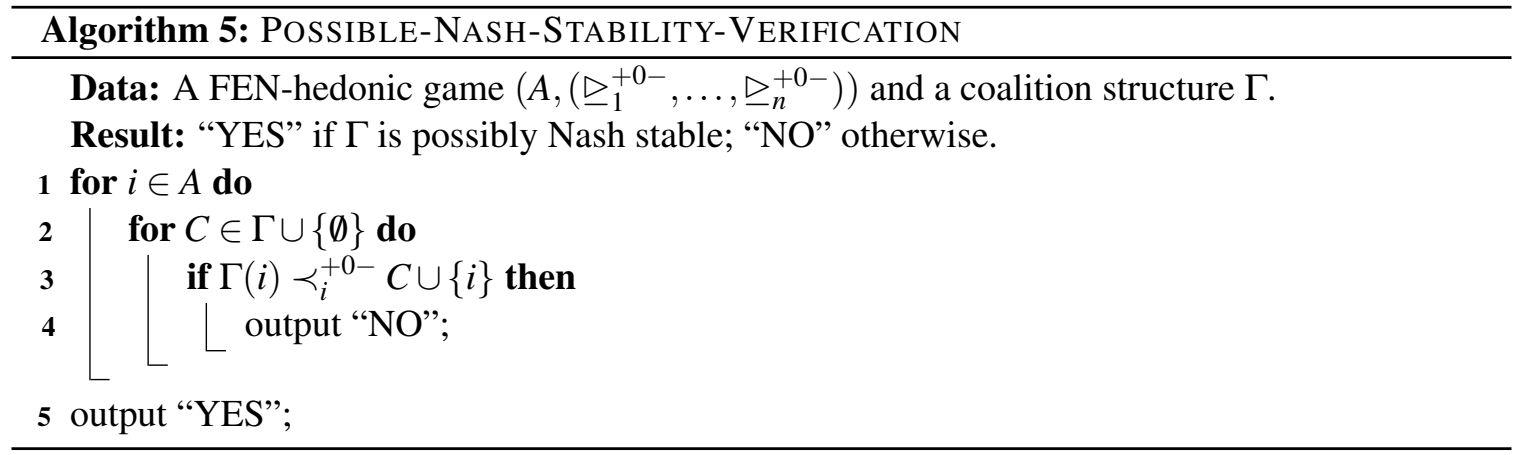

A coalition structure $\Gamma$ is possibly Nash stable if there is an extended profile $\left(\succeq_{1}, \ldots, \succeq_{n}\right) \in$ $\times_{i=1}^{n} \operatorname{Ext}\left(\succeq_{i}^{+0-}\right)$ such that for all agents $i \in A$ and all coalitions $C \in \Gamma \cup\{\emptyset\}$ we have $\Gamma(i) \succeq_{i} C \cup\{i\}$. 
Hence, Algorithm 5 checks if this condition possibly holds. Again, there are four cases for the relation between $\Gamma(i)$ and $C \cup\{i\}$ : We have either (1) $\Gamma(i) \succ_{i}^{+0-} C \cup\{i\}$, (2) $\Gamma(i) \sim_{i}^{+0-} C \cup\{i\}$, (3) $\Gamma(i) \prec_{i}^{+0-} C \cup\{i\}$, or (4) $\Gamma(i)$ and $C \cup\{i\}$ are incomparable.

In Cases (1), (2), and (3), the algorithm acts similarly as Algorithm 2. However, in Case (4), it is possible to set $\Gamma(i) \succeq_{i} C \cup\{i\}$ in the extension $\succeq_{i}$ of $\succeq_{i}^{+0-}$ such that Nash stability is not violated. Accordingly, the algorithm does not output "NO" in this case. Note that setting $\Gamma(i) \succeq_{i} C \cup\{i\}$ in Case (4) for multiple iterations can never result in a cyclic extension $\succeq_{i}$ because we just ensure that $\Gamma(i)$ is ranked better than all coalitions $C \in \Gamma \cup\{\emptyset\}$ that are incomparable to $\Gamma(i)$. The transitive closure of the resulting relation is the optimistic extension $\succeq_{i}^{+\Gamma(i)}$ as introduced in Section 3.4, which is acyclic by Proposition 16. Consequently, the choices made by Algorithm 5-each one individually allowed - to construct an extension can indeed be made simultaneously.

Basically, Algorithm 5 checks whether $\Gamma(i) \succeq_{i}^{+\Gamma(i)} C \cup\{i\}$ holds for all $i$ and all $C$, which is equivalent to $\Gamma$ being possibly Nash stable. Again, it is easy to see that the algorithm runs in polynomial time.

Next, we turn to verifying possible individual stability.

\section{Theorem 40 Possible-Individual-Stability-VerificAtion is in $\mathrm{P}$.}

Proof. Algorithm 6 solves the problem in polynomial time.

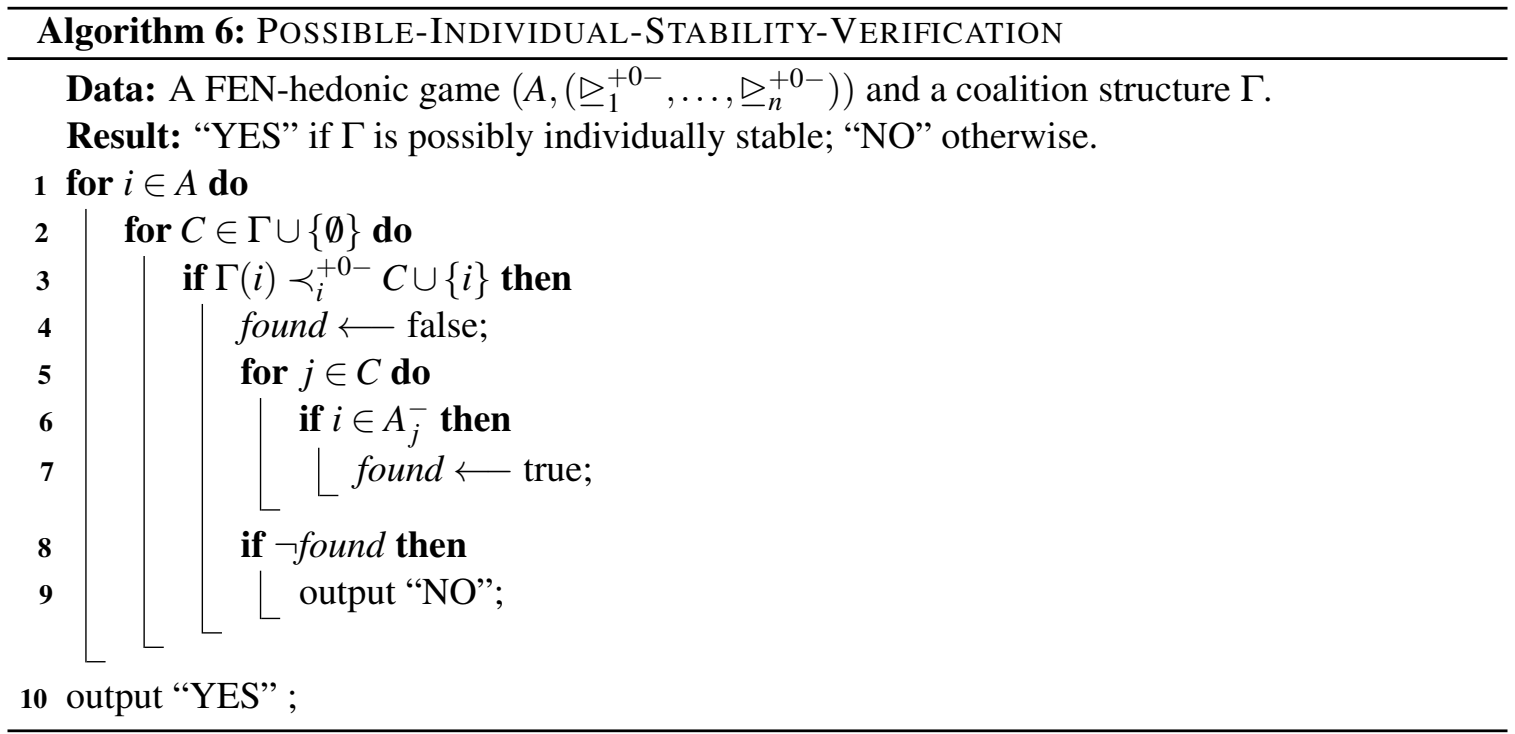

A coalition structure $\Gamma$ is possibly individually stable if there exists a profile $\left(\succeq_{1}, \ldots, \succeq_{n}\right)$ in $\times_{i=1}^{n} \operatorname{Ext}\left(\succeq_{i}^{+0-}\right)$ such that it holds for all agents $i \in A$ and all coalitions $C \in \Gamma \cup\{\emptyset\}$ that $\Gamma(i) \succeq_{i}$ $C \cup\{i\}$ or there exists an agent $j \in C$ who sees $i$ as an enemy. Therefore, Algorithm 6 checks if this condition is true. Again, it is easy to see that the algorithm runs in polynomial time.

Finally, we show that verifying possible contractually individual stability is easy.

Theorem 41 Possible-Contractually-Individual-STABILITY-VERIFICATION is in P. 


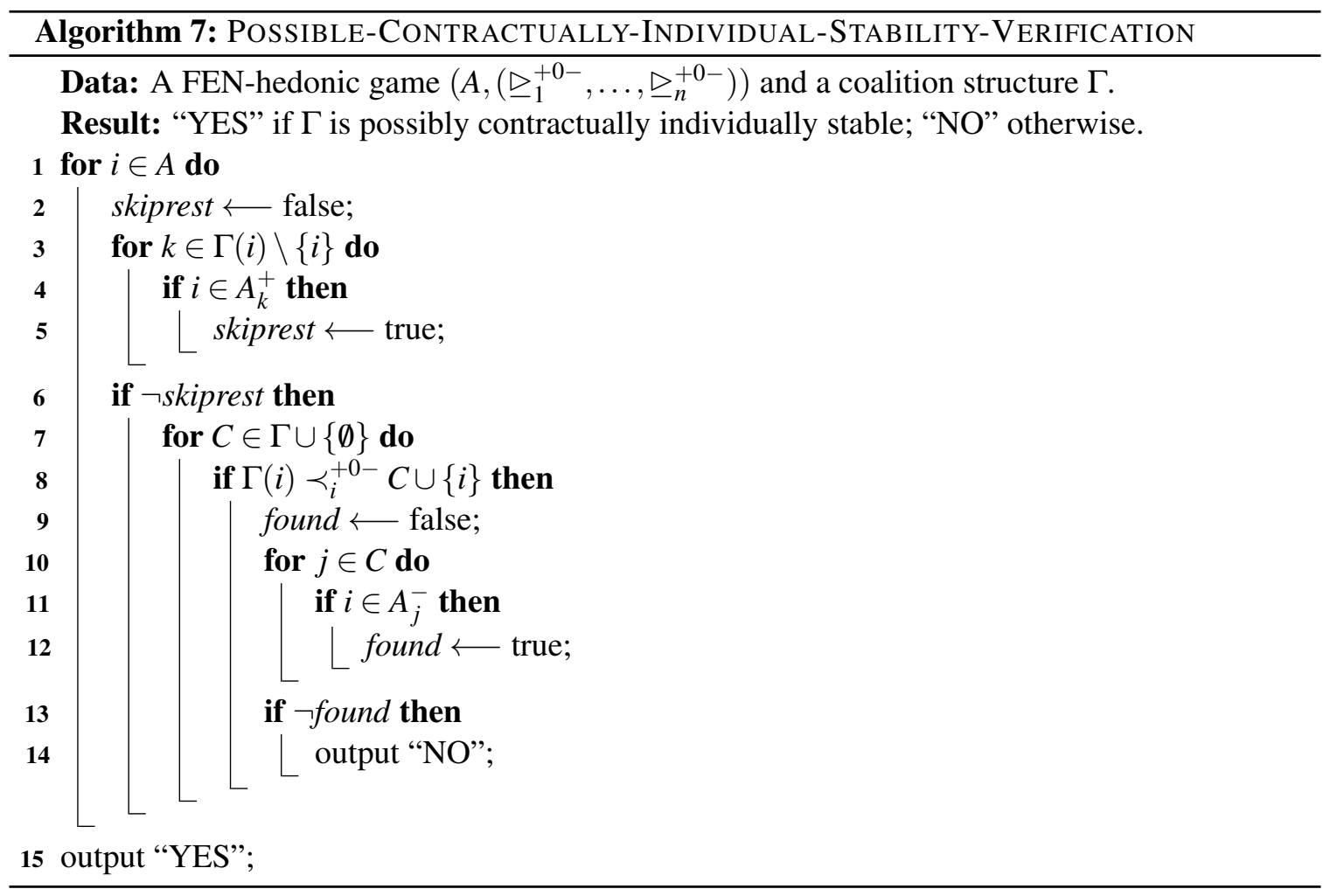

Proof. Algorithm 7 solves the problem in polynomial time.

As seen in the proof of Theorem 38, a coalition structure $\Gamma$ is contractually individually stable for a profile $\left(\succeq_{1}, \ldots, \succeq_{n}\right) \in \times_{i=1}^{n} \operatorname{Ext}\left(\succeq_{i}^{+0-}\right)$ exactly if for every agent $i \in A$, one of the following two conditions holds: (a) there exists an agent $k \in \Gamma(i)$ who sees $i$ as a friend; (b) for all coalitions $C \in \Gamma \cup\{\emptyset\}$, it holds that $\Gamma(i) \succeq_{i} C \cup\{i\}$ or there exists an agent $j \in C$ who sees $i$ as an enemy.

Hence, Algorithm 7 checks if (a) holds or $\succeq^{+0-}$ can be extended in such a way that (b) holds. Again, it is easy to see that the algorithm runs in polynomial time.

We conclude this section with an example showing coalition structures that are possibly or necessarily Nash, individually, or contractually individually stable.

Example 42 Consider the FEN-hedonic game $\left(A,\left(\unrhd_{1}^{+0-}, \ldots, \unrhd_{4}^{+0-}\right)\right)$ with $A=\{1,2,3,4\}$ and a coalition structure $\Gamma=\{\{1,2\},\{3,4\}\}$. The weak rankings with double threshold are:

$$
\begin{aligned}
& \unrhd_{1}^{+0-}=\left(2 \triangleright_{1}^{+} 3|\emptyset| 4 \quad\right), \\
& \unrhd_{2}^{+0-}=\left(\begin{array}{ll|l}
1 \triangleright_{2}^{+} 3 & 4 & \emptyset
\end{array}\right) \text {, } \\
& \unrhd_{3}^{+0-}=\left(\begin{array}{l|l|l}
1 & 4 & 2
\end{array}\right), \\
& \unrhd_{4}^{+0-}=\left(3 \quad|\emptyset| 1 \sim_{4}^{-} 2\right) .
\end{aligned}
$$

First, we can observe that none of 1, 2, and 4 wants to move to another coalition. However, coalitions $\{3,4\}$ and $\{1,2,3\}$ are incomparable for player 3 in the responsive extension. Hence, 3 possibly wants to move to coalition $\{1,2\}$. This means that $\Gamma$ is not necessarily Nash stable. This is 
detected by Algorithm 2 by testing if $\Gamma(3)=\{3,4\} \succeq_{3}^{+0-}\{1,2\} \cup\{3\}=C \cup\{3\}$. Since this is not the case, "NO" is output because player 3 possibly prefers $\{1,2,3\}$ to his coalition.

On the other hand, $\Gamma$ is possibly Nash stable because no player necessarily wants to move (not even player 3). This means that $\Gamma(i) \prec_{i}^{+0-} C \cup\{i\}$ doesn't hold for any $i \in A$ and $C \in \Gamma \cup\{\emptyset\}$, which is exactly what Algorithm 5 checks.

Since $\Gamma$ is possibly Nash stable, it is a fortiori possibly individually stable and possibly contractually individually stable. Necessary individual stability is not satisfied because 3 possibly wants to move to $\{1,2\}$ and she is not an enemy of 1 or 2 . Hence, she can possibly deviate without any member of the new coalition rejecting her. Finally, $\Gamma$ is necessarily contractually individually stable. This is so because 3 is the only player who possibly wants to move, but 4 doesn't want her to leave because 3 is a friend of 4's.

\subsection{Existence for Nash Stability, Individual Stability, and Contractually Individual Stability}

We now turn to the existence problems for Nash, individual, and contractually individual stability and first show that both possible and necessary existence are NP-complete for Nash stability.

\section{Theorem 43 Possible-NASH-STABILITY-EXISTEnCE is NP-complete.}

Proof. The problem belongs to NP, since we can verify possibly Nash stable coalition structures in polynomial time by Theorem 39 . Hence, we can nondeterministically guess a coalition structure and verify it in polynomial time.

NP-hardness can be shown via a polynomial-time many-one reduction from ExACT-COVERBY-ThreE-Sets (X3C), as defined in Section 2.3. Let $(B, \mathscr{S})$ be a given X3C instance, where $B$ has $3 m$ elements and $\mathscr{S}$ is a family of 3 -element subsets $S \subseteq B$. Without loss of generality, it can be assumed that $m \geq 2$ and each element in $B$ occurs at most three times in a set in $\mathscr{S}$. Given such an X3C instance, we construct the following game. ${ }^{4}$ The set $A$ consists of three types of players: $A=\left\{\alpha_{i} \mid 1 \leq i \leq 3 m-1\right\} \cup\left\{\beta_{b} \mid b \in B\right\} \cup\left(\bigcup_{S \in \mathscr{S}} Q_{S}\right)$, which we call chain, element, and set players and which we describe below together with their weak rankings with double threshold.

Chain players: For each $i, 1 \leq i \leq 3 m-1$, we have one chain player $\alpha_{i}$. Each of these players considers the "next" chain player to be her only friend, she is indifferent between the remaining chain players, and considers the other players to be enemies. Formally, for each $i$, $1 \leq i \leq 3 m-2$,

$$
\begin{aligned}
\unrhd_{\alpha_{i}}^{+0-} & \left.=\left(\alpha_{i+1}\left|\left\{\alpha_{j} \mid i \neq j \neq i+1\right\}\right| \text { other players }\right\}_{\sim}\right), \\
\unrhd_{\alpha_{3 m-1}}^{+0-} & \left.=\left(\emptyset\left|\left\{\alpha_{j} \mid j \neq 3 m-1\right\}\right| \text { \{other players }\right\}_{\sim}\right) .
\end{aligned}
$$

Element players: For each $b \in B$, there is one element player $\beta_{b}$. Each of these players considers all chain players to be her best ranked friends, followed by all set players (to be defined below) corresponding to sets $S \in \mathscr{S}$ containing $b$. The least preferred friends are the remaining element players. All other players are considered to be enemies, as there are no neutral players for $\beta_{b}$. Formally, for each $b \in B$,

\footnotetext{
${ }^{4}$ This construction is inspired by the construction of the proof that it is NP-hard to decide whether there exists a Nash stable coalition structure in an additively separable hedonic game (see Theorem 3 of Sung \& Dimitrov, 2010). However, several adjustments are needed to make the construction work to prove Theorem 43 .
} 


$$
\unrhd_{\beta_{b}}^{+0-}=\left(\left\{\alpha_{i} \mid 1 \leq i \leq 3 m-1\right\}_{\sim} \triangleright \bigcup_{\{S \mid b \in S\}} Q_{S \sim} \triangleright\left\{\beta_{b^{\prime}} \mid b^{\prime} \neq b\right\}_{\sim}|\emptyset|\{\text { other players }\}_{\sim}\right) .
$$

Set players: For each set $S \in \mathscr{S}$, we have one set $Q_{S}=\left\{\zeta_{S, k} \mid 1 \leq k \leq 3 m-2\right\}$ containing $3 m-2$ set players. The preferences of the set players in $Q_{S}$ are similar to the chain players. Each player considers the "next" set player to be her only friend and is indifferent between all remaining set players corresponding to the same set and those element players corresponding to elements in $S$. All other players are considered to be enemies. Formally, for each $S \in \mathscr{S}$ and for each $k, 1 \leq k \leq 3 m-3$,

$$
\begin{aligned}
\unrhd_{\zeta_{S, k}}^{+0-} & \left.=\left(\zeta_{S, k+1}\left|\left\{\zeta_{S, k^{\prime}} \mid k \neq k^{\prime} \neq k+1\right\} \cup\left\{\beta_{b} \mid b \in S\right\}\right| \text { \{other players }\right\}_{\sim}\right), \\
\unrhd_{\zeta_{S, 3 m-2}}^{+0-} & \left.=\left(\emptyset\left|\left\{\zeta_{S, k^{\prime}} \mid k^{\prime} \neq 3 m-2\right\} \cup\left\{\beta_{b} \mid b \in S\right\}\right| \text { \{other players }\right\}_{\sim}\right) .
\end{aligned}
$$

Moreover, for each $S \in \mathscr{S}$, we denote the union of $Q_{S}$ and all element players belonging to $S$ with $P_{S}=\left\{\beta_{b} \mid b \in S\right\} \cup Q_{S}$. This profile can be constructed in polynomial time, since there are $n \leq 3 m+3 m+3 m \cdot(3 m-2)=9 m^{2}$ players, and each player's preference can be written in time linear in $n$. Figure 6 gives a visualization of the profile. ${ }^{5}$

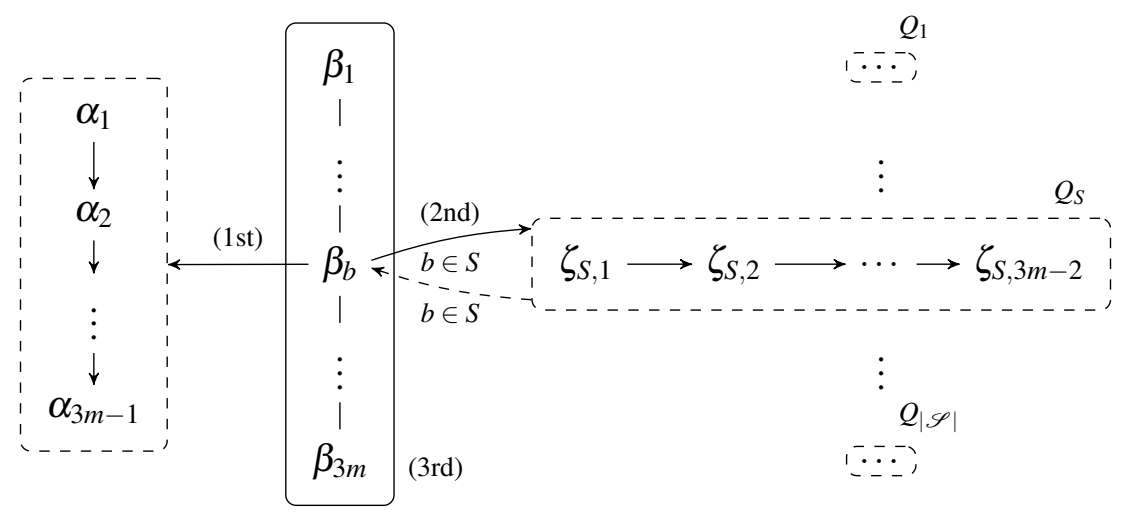

Figure 6: Network of friends for the construction in the proof of Theorem 43

We now show that $(B, \mathscr{S})$ is in $\mathrm{X} 3 \mathrm{C}$ if and only if there exists a possibly Nash stable coalition structure in the PR-extension of the constructed game.

Only if: Assume there exists a solution $\mathscr{S}^{\prime}$ for $(B, \mathscr{S})$. Consider the coalition structure $\Gamma=$ $\left\{\left\{\alpha_{i} \mid 1 \leq i \leq 3 m-1\right\}\right\} \cup\left\{P_{S} \mid S \in \mathscr{S}^{\prime}\right\} \cup\left\{Q_{S} \mid S \notin \mathscr{S}^{\prime}\right\}$. By a close look at all (possibly empty) coalitions in $\Gamma$ it can be seen that no $\alpha_{i}, 1 \leq i \leq 3 m-1$, and no $\zeta_{S, k}, S \in \mathscr{S}, 1 \leq k \leq 3 m-2$, wants to move, and each $\beta_{b}, b \in B$, possibly does not want to move due to the number of friends; thus $\Gamma$ is possibly Nash stable.

If: Assume there is a possibly Nash stable coalition structure $\Gamma$. We start with the assignment of the chain players $\alpha_{i}$ to the coalitions in $\Gamma$. Let $C=\left\{\alpha_{1}, \ldots, \alpha_{3 m-1}\right\}$ be the set containing all the

\footnotetext{
${ }^{5}$ In Figure 6 and in the upcoming Figures 7 and 5, a solid line/arrow represents a friendship relation (with priorities if required) and a dashed line/arrow stands for a neutral or a friendship relation. Also note that, for the sake of readability, we identify player names with vertex names in illustrations of networks.
} 
chain players. We will argue that $C$ has to be a coalition in $\Gamma$ : First, $\Gamma$ cannot contain any coalition that contains a strict subset of $C=\left\{\alpha_{1}, \ldots, \alpha_{3 m-1}\right\}$ because, as player $\alpha_{3 m-2}$ 's only friend is $\alpha_{3 m-1}$, she will always want to move to the coalition $\alpha_{3 m-1}$ is contained in. Hence, any coalition structure assigning these two players to different coalitions is not possibly Nash stable. For the same reasons, $\alpha_{3 m-3}$ will always follow $\alpha_{3 m-2}$, and so on; thus no coalition structure $\Delta$ with $\Delta\left(\alpha_{i}\right) \neq \Delta\left(\alpha_{i+1}\right), 1 \leq$ $i \leq 3 m-2$, is possibly Nash stable. Second, as soon as any other player is added to $C$, player $\alpha_{3 m-1}$ necessarily prefers being alone to being in $C$. Thus $\Gamma$ does not contain any strict superset of $C$. Therefore, it holds that $C \in \Gamma$. With an analogous argumentation we can show that for each $S \in \mathscr{S}$ the corresponding set players in $Q_{S}$ have to be in the same coalition for $\Gamma$ to be possibly Nash stable. It holds that for each $S \in \mathscr{S}$, each $\zeta_{S, k}$ follows $\zeta_{S, k+1}$ sequentially, $k=3 m-3, \ldots, 1$, to a superset $D_{S}$ of $Q_{S}$. $D_{S}$ cannot contain any set players from other sets, that is, any $\zeta_{S^{\prime}, k^{\prime}}$ with $S^{\prime} \neq S$, $1 \leq k^{\prime} \leq 3 m-2$. Furthermore, $D_{S}$ cannot contain any element player $\beta_{b}$ with $b \notin S$, since $\zeta_{S, 3 m-2}$ is indifferent between everyone but her enemies and will deviate.

This leaves us only the following combinations to consider: For each $S \in \mathscr{S}, \Gamma$ contains the coalition $D_{S}=Q_{S} \cup R_{S}$, where $R_{S} \subseteq\left\{\beta_{b} \mid b \in S\right\}$. If $R_{S}$ contains one element player $\beta_{b}$, then there are fewer friends of $\beta_{b}$ in coalition $D_{S}$ than in $C$. If $R_{S}$ contains two element players, $\beta_{b}$ and $\beta_{b^{\prime}}$, then both coalitions $C$ and $D_{S}$ contain the same number of $\beta_{b}$ 's friends. Since both coalitions contain at most $3 m-1$ friends and enemies, and each friend in the first coalition is ranked higher than one in the latter, $\beta_{b}$ necessarily prefers $C \cup\left\{\beta_{b}\right\}$ to $D_{S}$. Hence, either $D_{S}=Q_{S}$ or $D_{S}=P_{S}$. If an element player $\beta_{b}$ was alone with other element players $\beta_{b^{\prime}}$, which are in turn not in $P_{S}$, she would be with at most $3 m-1$ friends, and would rather move to $C$ with the same number of, but higher ranked, friends. This implies that for each $b \in B$, there exists an $S \in \mathscr{S}$ such that $\Gamma\left(\beta_{b}\right)=P_{S}$, so the coalitions the element players have to be assigned to induce an exact cover of $B$ in $\mathscr{S}$.

With a similar construction, we can show the following.

\section{Theorem 44 Necessary-NASH-STABILITY-EXISTENCE is NP-complete.}

Proof. The problem belongs to NP, since it can be verified in polynomial time in the number of players whether a nondeterministically chosen coalition structure is necessarily Nash stable by Theorem 36. NP-hardness can be shown similarly to the proof of Theorem 43. Given an X3C instance $(B, \mathscr{S})$, we again construct a game with three types of players: $A=\left\{\alpha_{i} \mid 1 \leq i \leq 3 m\right\} \cup\left\{\beta_{b} \mid\right.$ $b \in B\} \cup\left\{\zeta_{S, k} \mid S \in \mathscr{S}, 1 \leq k \leq 3 m-2\right\}$. In comparison to the proof of Theorem 43, we have an additional chain player, $\alpha_{3 m}$, and we change the order of friends for the element players $\beta_{b}, b \in B$.

Chain players: For each $i, 1 \leq i \leq 3 m$, we have one chain player $\alpha_{i}$. Each of these players considers the "next" chain player to be her only friend, she is indifferent between the remaining chain players, and considers all other players to be enemies. Formally, for each $i, 1 \leq i \leq 3 m-1$,

$$
\begin{aligned}
& \left.\unrhd_{\alpha_{i}}^{+0-}=\left(\alpha_{i+1}\left|\left\{\alpha_{j} \mid i \neq j \neq i+1\right\}\right| \text { \{other players }\right\}_{\sim}\right), \\
& \left.\unrhd_{\alpha_{3 m}}^{+0-}=\left(\emptyset\left|\left\{\alpha_{j} \mid j \neq 3 m\right\}\right| \text { \{other players }\right\}_{\sim}\right) .
\end{aligned}
$$

Element players: For each $b \in B$, there is one element player $\beta_{b}$. Each of these players considers those set players (to be defined below) corresponding to sets $S \in \mathscr{S}$ containing $b$ to be her best friends, followed by all chain players and the remaining element players. There are no neutral players for $\beta_{b}$, so all other players are enemies. Formally, for each $b \in B$, 


$$
\unrhd_{\beta_{b}}^{+0-}=\left(\bigcup_{\{S \mid b \in S\}} Q_{S \sim} \triangleright\left\{\alpha_{i} \mid 1 \leq i \leq 3 m-1\right\}_{\sim} \triangleright\left\{\beta_{b^{\prime}} \mid b^{\prime} \neq b\right\}_{\sim}|\emptyset|\{\text { other players }\}_{\sim}\right) .
$$

Set players: For each set $S \in \mathscr{S}$, we have one set $Q_{S}=\left\{\zeta_{S, k} \mid 1 \leq k \leq 3 m-2\right\}$ containing $3 m-2$ set players. For a fixed set $S \in \mathscr{S}$, the preferences of the set players in $Q_{S}$ are similar to those of the chain players. Each player considers the "next" set player to be her only friend and is indifferent between all remaining set players corresponding to the same set and those element players corresponding to the elements in $S$. All other players are considered to be enemies. Formally, for each $S \in \mathscr{S}$ and for each $k, 1 \leq k \leq 3 m-3$,

$$
\begin{aligned}
\unrhd_{\zeta_{S, k}}^{+0-} & \left.=\left(\zeta_{S, k+1}\left|\left\{\zeta_{S, k^{\prime}} \mid k \neq k^{\prime} \neq k+1\right\} \cup\left\{\beta_{b} \mid b \in S\right\}\right| \text { \{other players }\right\}_{\sim}\right), \\
\unrhd_{\zeta_{S, 3 m-2}}^{+0-} & \left.=\left(\emptyset\left|\left\{\zeta_{S, k^{\prime}} \mid k^{\prime} \neq 3 m-2\right\} \cup\left\{\beta_{b} \mid b \in S\right\}\right| \text { \{other players }\right\}_{\sim}\right) .
\end{aligned}
$$

Again, we denote for each $S \in \mathscr{S}$ with $P_{S}=Q_{S} \cup\left\{\beta_{b} \mid b \in S\right\}$ the set containing all set players corresponding to $S$ and those element players who are corresponding to the elements in $S$.

We show that $(B, \mathscr{S})$ is in $\mathrm{X} 3 \mathrm{C}$ if and only if there exists a necessarily Nash stable coalition structure in the polarized responsive extension of the constructed game.

Only if: Assume that $\mathscr{S}^{\prime}$ is a solution for $(B, \mathscr{S})$. Let $C=\left\{\alpha_{1}, \ldots, \alpha_{3 m}\right\}$ and consider the coalition structure $\Gamma=\{C\} \cup\left\{P_{S} \mid S \in \mathscr{S}^{\prime}\right\} \cup\left\{Q_{S} \mid S \notin \mathscr{S}^{\prime}\right\}$. No chain player $\alpha_{i}, 1 \leq i \leq 3 m$, and no set player $\zeta_{S, k}, S \in \mathscr{S}, 1 \leq k \leq m$, wants to leave her coalition. Each element player $\beta_{b}, b \in B$, now necessarily prefers being in $P_{S}$ to joining any other existing or the empty coalition. Thus $\Gamma$ is necessarily Nash stable.

If: Let $\Gamma$ be a necessarily Nash stable coalition structure. Analogously to the argumentation in the proof of Theorem 43, the coalition $C=\left\{\alpha_{1}, \alpha_{2}, \ldots, \alpha_{3 m}\right\}$ has to be in $\Gamma$ (as argued in the proof of Theorem 43), because of the preferences of the chain players $\alpha_{i}, 1 \leq i \leq 3 \mathrm{~m}$. Furthermore, the coalition $D_{S}=Q_{S} \cup R_{S}$, where $R_{S} \subseteq\left\{\beta_{b} \mid b \in S\right\}$, has to be in $\Gamma$ for each $S \in \mathscr{S}$ (as argued in the proof of Theorem 43). The set $R_{S}$ cannot contain one or two players, since otherwise one element player $\beta_{b} \in R_{S}$ would possibly prefer moving to coalition $C$, which would contradict the assumption of necessary Nash stability of $\Gamma$. Consequently, either the coalition $D_{S}=Q_{S}$ or the coalition $D_{S}=P_{S}$ has to be in $\Gamma$ for each $S \in \mathscr{S}$. An element player $\beta_{b}$ outside of $P_{S}$ in $\Gamma$ would also imply a possible deviation to $C \cup\left\{\beta_{b}\right\}$. Thus all element players $\beta_{b}$ with $b \in B$ are covered by disjoint sets $P_{S}$ in $\Gamma$; hence, $\left\{S \mid P_{S} \in \Gamma\right\}$ induces a solution to the $\mathrm{X} 3 \mathrm{C}$ instance $(B, \mathscr{S})$.

We now turn to individual stability and contractually individual stability. We first show that possible existence is easy for contractually individual stability. Afterwards, we will show that necessary existence is NP-complete for individual stability.

Theorem 45 There always exists a possibly contractually individually stable coalition structure in a FEN-hedonic game.

Proof. This follows from Theorem 30 (which says that a possibly Pareto optimal coalition structure always exists in a FEN-hedonic game), together with the fact that Pareto optimality implies contractually individual stability.

We will now show that deciding whether, given a FEN-hedonic game, there exists a necessarily individually stable coalition structure is NP-complete. To this end, Construction 46 is needed, and 
we briefly explain the ideas behind it. We will provide a polynomial-time many-one reduction from NECESSARY-NASH-STABILITY-EXISTENCE (NNSE, for short), which is NP-complete by Theorem 44. We take a FEN-hedonic game $H$ that is an instance of NNSE and construct another FENhedonic game $H^{\prime}$, which is an instance of NECESSARY-INDIVIDUAL-STABILITY-EXISTENCE, such that there exists a necessarily individually stable coalition structure for $H^{\prime}$ if and only if there exists a necessarily Nash stable coalition structure for $H$.

In the upcoming construction, we define so-called clone players which have the same preferences as the original players (from $H$ ) but unlike the original players are not the enemy of any other player. By this trick we eliminate the possibility that other players can prevent the deviation of a clone player. Furthermore, we include so-called structure players to ensure that every necessarily individually stable coalition structure has to satisfy a certain form. Finally, so-called friend and enemy players help the structure players to fulfill their purpose.

Construction 46 Let $H=\left(A, \unrhd^{+0-}\right)$ be a FEN-hedonic game with $A=\{1, \ldots, n\}, \unrhd^{+0-}=\left(\unrhd_{1}^{+0-}\right.$, $\left.\ldots, \unrhd_{n}^{+0-}\right)$ and $\unrhd_{i}^{+0-}=\left(\unrhd_{i}^{+}\left|A_{i}^{0}\right| \unrhd_{i}^{-}\right)$for every $i \in A$, where $\unrhd_{i}^{+}$is the weak order over the set of $i$ 's friends $A_{i}^{+}$and $\unrhd_{i}^{-}$is the weak order over the set of $i$ 's enemies $A_{i}^{-}$. We now construct a FENhedonic game $H^{\prime}$ in polynomial time. Let $H^{\prime}=\left(A^{\prime}, \unrhd^{+0-\prime}\right)$ be a FEN-hedonic game with $A^{\prime}=$ $A \cup$ Clone $\cup$ Structure $\cup$ Friend $_{A} \cup$ Friend $_{B} \cup$ Enemy, Clone $=\left\{c_{1}, \ldots, c_{n}\right\}$, Structure $=\left\{s_{1}, \ldots, s_{n}\right\}$, Friend $_{A}=\left\{a_{1}, \ldots, a_{n}\right\}$, Friend ${ }_{B}=\left\{b_{1}, \ldots, b_{n}\right\}$, and Enemy $=\left\{e_{1}, \ldots, e_{n}\right\}$. Furthermore, let $\unrhd^{+0-\prime}=$ $\left(\unrhd_{1}^{+0-\prime}, \ldots, \unrhd_{e_{n}}^{+0-\prime}\right)$ and, for $1 \leq i \leq n$, let

$$
\begin{aligned}
& \unrhd_{i}^{+0-\prime}=\left(\unrhd_{i}^{+\prime}\left|A_{i}^{0 \prime}\right| \unrhd_{i}^{-\prime}\right)=( \\
& \unrhd_{c_{i}}^{+0-\prime}=\left(\unrhd_{c_{i}}^{+\prime}\left|A_{c_{i}}^{0 \prime}\right| \unrhd_{c_{i}}^{-\prime}\right)=\left(\unrhd_{i}^{+}\right. \\
& \mid A^{\prime} \backslash\{i\} \\
& \unrhd_{s_{i}}^{+0-\prime}=\left(\unrhd_{s_{i}}^{+\prime}\left|A_{s_{i}}^{0 \prime}\right| \unrhd_{s_{i}}^{-\prime}\right)=\left(i \sim_{s_{i}} c_{i} \triangleright_{s_{i}} a_{i} \sim_{s_{i}} b_{i}\right. \\
& \mid A^{\prime} \backslash\left(A_{i}^{+} \cup A_{i}^{-} \cup\left\{c_{i}\right\}\right) \\
& \text { | ), } \\
& \unrhd_{a_{i}}^{+0-\prime}=\left(\unrhd_{a_{i}}^{+\prime}\left|A_{a_{i}}^{0 \prime}\right| \unrhd_{a_{i}}^{-\prime}\right)=\left(b_{i}\right. \\
& \left|A^{\prime} \backslash\left\{i, c_{i}, s_{i}, a_{i}, b_{i}, e_{i}\right\} \quad\right| e_{i} \\
& \mid A^{\prime} \backslash\left\{a_{i}, b_{i}\right\} \\
& \unrhd_{b_{i}}^{+0-\prime}=\left(\unrhd_{b_{i}}^{+\prime}\left|A_{b_{i}}^{0 \prime}\right| \unrhd_{b_{i}}^{-\prime}\right)=\left(e_{i}\right. \\
& \mid A^{\prime} \backslash\left\{b_{i}, e_{i}\right\} \\
& \unrhd_{e_{i}}^{+0-\prime}=\left(\unrhd_{e_{i}}^{+\prime}\left|A_{e_{i}}^{0 \prime}\right| \unrhd_{e_{i}}^{-\prime}\right)=( \\
& \mid A^{\prime} \backslash\left(A \cup\left\{e_{i}\right\}\right)
\end{aligned}
$$

This construction can obviously be done in polynomial time.

Example 47 Consider the FEN-hedonic game $\left(A,\left(\unrhd_{1}^{+0-}, \unrhd_{2}^{+0-}, \unrhd_{3}^{+0-}\right)\right)$ with $A=\{1,2,3\}$ and a coalition structure $\Gamma=\{\{1\},\{2,3\}\}$. The weak rankings with double threshold are:

$$
\begin{aligned}
& \unrhd_{1}^{+0-}=\left(\begin{array}{lll}
2 \triangleright_{1}^{+} 3 & \emptyset & \emptyset
\end{array}\right), \\
& \unrhd_{2}^{+0-}=\left(\begin{array}{l|l|l}
3 & 1 & \emptyset
\end{array}\right), \\
& \unrhd_{3}^{+0-}=\left(\emptyset \quad|\emptyset| 2 \triangleright_{3}^{-} 1\right) .
\end{aligned}
$$

Note that $\Gamma$ is not possibly or necessarily Nash stable because 1 wants to move to $\{2,3\} . \Gamma$ is not possibly or necessarily individually stable because 3 wants to move to the empty coalition $\emptyset$ and there's no player in this empty coalition who could reject $3 . \Gamma$ is possibly and necessarily contractually individually stable because there are two players who would like to deviate, 1 and 3 , but for both there is another player prohibiting the deviation. 1 wants to move to $\{2,3\}$ but 3 doesn't want 1 to join. 3 wants to move to $\emptyset$ but 2 doesn't want her to leave $\{2,3\}$. 
The FEN-hedonic game $H^{\prime}=\left(A^{\prime}, \unrhd^{+0-\prime}\right)$ as stated in Construction 46 consists of $A^{\prime}=\{1,2,3\} \cup$ $\left\{c_{1}, c_{2}, c_{3}\right\} \cup\left\{s_{1}, s_{2}, s_{3}\right\} \cup\left\{a_{1}, a_{2}, a_{3}\right\} \cup\left\{b_{1}, b_{2}, b_{3}\right\} \cup\left\{e_{1}, e_{2}, e_{3}\right\}, \unrhd^{+0-\prime}=\left(\unrhd_{1}^{+0-\prime}, \ldots, \unrhd_{e_{3}}^{+0-\prime}\right)$ and for $i \in\{1,2,3\}$ the preferences are

\begin{tabular}{|c|c|c|}
\hline$\unrhd_{i}^{+0-1}=($ & $\mid A^{\prime} \backslash\{i\}$ & $\mid$ \\
\hline$\unrhd_{c_{1}}^{+0-1}=\left(2 \triangleright_{c_{1}}^{+} 3\right.$ & $\left.\mid A^{\prime} \backslash\left\{2,3, c_{1}\right\}\right)$ & \\
\hline$\unrhd_{c_{2}}^{+0-1}=(3$ & $\left.\mid A^{\prime} \backslash\left\{3, c_{2}\right\}\right)$ & 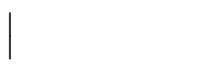 \\
\hline$\unrhd_{c_{3}}^{+0-1}=($ & $\left.\mid A^{\prime} \backslash\left\{1,2, c_{3}\right\}\right)$ & $\mid 2 \triangleright_{c_{3}}^{-} 1$ \\
\hline$\unrhd_{s_{i}}^{+0-\prime}=\left(i \sim_{s_{i}} c_{i} \triangleright_{s_{i}} a_{i} \sim_{s_{i}} b_{i}\right.$ & $\mid A^{\prime} \backslash\left\{i, c_{i}, s_{i}, a_{i}, b_{i}, e_{i}\right\}$ & $e_{i}$ \\
\hline$\unrhd_{a_{i}}^{+0-\prime}=\left(b_{i}\right.$ & $\mid A^{\prime} \backslash\left\{a_{i}, b_{i}\right\}$ & $\mid$ \\
\hline$\unrhd_{b_{i}}^{+0-1}=\left(e_{i}\right.$ & $\mid A^{\prime} \backslash\left\{b_{i}, e_{i}\right\}$ & 1 \\
\hline$\unrhd_{e_{i}}^{+0-1}=($ & $\mid A^{\prime} \backslash\left\{1,2,3, e_{i}\right\}$ & $\mid 1 \sim_{e_{i}} 2 \sim_{e_{i}} 3$ \\
\hline
\end{tabular}

We will later consider the coalition structure $\Gamma^{\prime}=\left\{D_{C}, E_{C} \mid C \in \Gamma\right\}$ with $D_{C}=\left\{j, c_{j}, s_{j} \mid j \in\right.$ $C\}$ and $E_{C}=\left\{a_{j}, b_{j}, e_{j} \mid j \in C\right\}$. Here, this means $\Gamma^{\prime}=\left\{\left\{1, c_{1}, s_{1}\right\},\left\{a_{1}, b_{1}, e_{1}\right\},\left\{2, c_{2}, s_{2}, 3, c_{3}, s_{3}\right\}\right.$, $\left.\left\{a_{2}, b_{2}, e_{2}, a_{3}, b_{3}, e_{3}\right\}\right\}$. Due to the construction of $H^{\prime}$, it holds that $\Gamma$ not being necessarily Nash stable implies that $\Gamma^{\prime}$ is not necessarily individually stable. Since 1 wants to move to $\{2,3\}$ in $H$, the corresponding clone player $c_{1}$ wants to move to $\left\{2, c_{2}, s_{2}, 3, c_{3}, s_{3}\right\}$ in $H^{\prime}$. And since there is no player who has $c_{1}$ as an enemy, no player could prevent the deviation.

Theorem 48 NecESSARY-INDIVIDUAL-STABILITY-EXISTENCE (NISE, for short) is NP-complete.

Proof. To see that NISE is in NP, let the FEN-hedonic game $H=\left(A, \unrhd^{+0-}\right)$ be a given instance. We nondeterministically guess a coalition structure $\Gamma \in \mathscr{C}_{\left(A, \triangleright^{+0-}\right)}$ that might be a solution for this instance. Then we check whether $\Gamma$ indeed is a solution, i.e., whether $\Gamma$ necessarily satisfies individual stability. This is possible in polynomial time by Theorem 37.

We show NP-hardness of NISE by providing a polynomial-time many-one reduction from NNSE. To do so, we consider the FEN-hedonic games $H$ and $H^{\prime}$ as defined in Construction 46, where $H$ is considered to be an instance of NNSE and $H^{\prime}$ an instance of NISE. Obviously, the construction of $H^{\prime}$ can be done in polynomial time.

We will now show that

$$
H \in \mathrm{NNSE} \Longleftrightarrow H^{\prime} \in \text { NISE. }
$$

From left to right, assume that $H \in \mathrm{NNSE}$. This means that there exists a coalition structure $\Gamma \in \mathscr{C}_{\left(A, \unrhd^{+0-}\right)}$ such that for every extended profile $\left(\succeq_{1}, \ldots, \succeq_{n}\right) \in \times_{i=1}^{n} \operatorname{Ext}\left(\succeq_{i}^{+0-}\right)$, it holds that $(\forall i \in A)(\forall C \in \Gamma \cup\{\emptyset\})\left[\Gamma(i) \succeq_{i} C \cup\{i\}\right]$. Since this relation holds for every extended profile, it also has to hold for $\left(\succeq_{1}^{+0-}, \ldots, \succeq_{n}^{+0-}\right)$. Hence, we have

$$
(\forall i \in A)(\forall C \in \Gamma \cup\{\emptyset\})\left[\Gamma(i) \succeq_{i}^{+0-} C \cup\{i\}\right] .
$$

We will now show that $H^{\prime} \in$ NISE, i.e., that there is a coalition structure $\Gamma^{\prime} \in \mathscr{C}_{\left(A^{\prime}, \unrhd^{+0-\prime}\right)}$ such that

$$
\left(\forall i \in A^{\prime}\right)\left(\forall C^{\prime} \in \Gamma^{\prime} \cup\{\emptyset\}\right)\left[\Gamma^{\prime}(i) \succeq_{i}^{+0-\prime} C^{\prime} \cup\{i\} \vee\left(\exists j \in C^{\prime}\right)\left[i \in A_{j}^{-\prime}\right]\right]
$$


We consider the coalition structure $\Gamma^{\prime}=\left\{D_{C}, E_{C} \mid C \in \Gamma\right\}$ with $D_{C}=\left\{j, c_{j}, s_{j} \mid j \in C\right\}$ and $E_{C}=$ $\left\{a_{j}, b_{j}, e_{j} \mid j \in C\right\}$. It then holds for all $i \in A$ that $\Gamma^{\prime}(i)=\Gamma^{\prime}\left(c_{i}\right)=\Gamma^{\prime}\left(s_{i}\right)=\left\{j, c_{j}, s_{j} \mid j \in \Gamma(i)\right\}$ and $\Gamma^{\prime}\left(a_{i}\right)=\Gamma^{\prime}\left(b_{i}\right)=\Gamma^{\prime}\left(e_{i}\right)=\left\{a_{j}, b_{j}, e_{j} \mid j \in \Gamma(i)\right\}$.

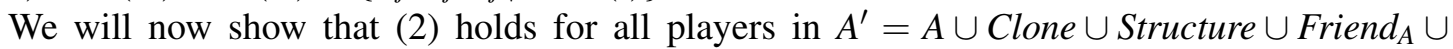
Friend $_{B} \cup$ Enemy. First, consider the players $i \in A$. It holds that $\Gamma^{\prime}(i) \succeq_{i}^{+0-\prime} C^{\prime} \cup\{i\}$ for all $C^{\prime} \in \Gamma^{\prime} \cup\{\emptyset\}$ because $i$ doesn't have any friends or enemies and therefore is indifferent between any two coalitions. Hence, (2) is satisfied for all $i \in A$ and all $C^{\prime} \in \Gamma^{\prime} \cup\{\emptyset\}$.

Next, consider the clone players $c_{i}$. For all $D_{C}=\left\{j, c_{j}, s_{j} \mid j \in C\right\} \in \Gamma^{\prime}$, it holds that $\Gamma^{\prime}\left(c_{i}\right)=$ $\left\{j, c_{j}, s_{j} \mid j \in \Gamma(i)\right\} \succeq_{c_{i}}^{+0-\prime}\left\{j, c_{j}, s_{j} \mid j \in C\right\} \cup\left\{c_{i}\right\}=D_{C} \cup\left\{c_{i}\right\}$ if and only if $\Gamma(i) \cup\left\{c_{i}\right\} \succeq_{c_{i}}^{+0-\prime} C \cup$ $\left\{c_{i}\right\}$ because $c_{i}$ is neutral to all other clone players and all structure players. This in turn is equivalent to $\Gamma(i) \backslash\{i\} \cup\left\{c_{i}\right\} \succeq_{c_{i}}^{+0-1} C \cup\left\{c_{i}\right\}$ because $c_{i}$ is neutral to $i$. Since $c_{i}$ has the same friends, order over friends, enemies, and order over enemies as player $i$ has in $H$, the last preference relation is equivalent to $\Gamma(i) \succeq_{i}^{+0-} C \cup\{i\}$, which holds by assumption, see Equation (1). Hence, (2) is satisfied for all $c_{i}$ and $D_{C} \in \Gamma^{\prime}$.

Now, consider all $E_{C}=\left\{a_{j}, b_{j}, e_{j} \mid j \in C\right\} \in \Gamma^{\prime}$. Again, $\Gamma^{\prime}\left(c_{i}\right)=\left\{j, c_{j}, s_{j} \mid j \in \Gamma(i)\right\} \succeq_{c_{i}}^{+0-\prime}$ $\left\{a_{j}, b_{j}, e_{j} \mid j \in C\right\} \cup\left\{c_{i}\right\}=E_{C} \cup\left\{c_{i}\right\}$ is equivalent to $\Gamma(i) \backslash\{i\} \cup\left\{c_{i}\right\} \succeq_{c_{i}}^{+0-\prime}\left\{c_{i}\right\}$ by removing all neutral players. This is equivalent to $\Gamma(i) \succeq_{i}^{+0-}\{i\}$, which holds by Equation (1). It is easy to see that the same argumentation is possible for the empty coalition $\emptyset$. Hence, (2) is satisfied for all $c_{i} \in$ Clone and all $C^{\prime} \in \Gamma^{\prime} \cup\{\emptyset\}$.

We now turn to the structure players $s_{i} . \Gamma^{\prime}\left(s_{i}\right)=\left\{j, c_{j}, s_{j} \mid j \in \Gamma(i)\right\}$ contains $s_{i}$ 's two best friends, $i$ and $c_{i}$, and no enemy. Every other coalition can only contain at most two other friends of $s_{i}$, namely $a_{i}$ and $b_{i}$, which are ranked lower than $i$ and $c_{i}$. Hence, $s_{i}$ prefers $\Gamma^{\prime}\left(s_{i}\right)$ to every other coalition in $\Gamma^{\prime} \cup\{\emptyset\}$ and (2) is satisfied for all $s_{i} \in$ Structure.

For all $a_{i} \in$ Friend $_{A}$, it holds that $\Gamma^{\prime}\left(a_{i}\right)=\left\{a_{j}, b_{j}, e_{j} \mid j \in \Gamma(i)\right\} \succeq_{a_{i}}^{+0-\prime} C^{\prime} \cup\left\{a_{i}\right\}$ for every coalition $C^{\prime} \in \Gamma^{\prime} \cup\{\emptyset\}$ because $\Gamma^{\prime}\left(a_{i}\right)$ contains $b_{i}\left(a_{i}\right.$ 's only friend) and no enemies. Therefore, (2) holds for all $a_{i} \in$ Friend $_{A}$. Analogously, (2) also holds for all $b_{i} \in$ Friend $_{B}$. Finally, consider the enemy players $e_{i}$. Since $e_{i}$ has no friends and $\Gamma^{\prime}\left(e_{i}\right)=\left\{a_{j}, b_{j}, e_{j} \mid j \in \Gamma(i)\right\}$ doesn't contain any enemies of $e_{i}$, it holds that $\Gamma^{\prime}\left(e_{i}\right) \succeq_{e_{i}}^{+0-\prime} C^{\prime} \cup\left\{e_{i}\right\}$ for every $C^{\prime} \in \Gamma^{\prime} \cup\{\emptyset\}$. So, (2) also holds for all $e_{i} \in$ Enemy.

Thus (2) is satisfied for all players in $A^{\prime}$ and all $C^{\prime} \in \Gamma^{\prime} \cup\{\emptyset\}$, which means that $\Gamma^{\prime}$ is necessarily individually stable for $H^{\prime}$ and $H^{\prime} \in$ NISE.

From right to left, assume that $H^{\prime} \in$ NISE. Then, there is a $\Gamma^{\prime} \in \mathscr{C}_{\left(A^{\prime}, \unrhd^{+0-\prime}\right)}$ such that (2) holds. Consider such a coalition structure $\Gamma^{\prime}$. We will now show that $\Gamma^{\prime}$ necessarily needs to be of the following form because (2) couldn't hold otherwise:

$$
\begin{aligned}
& \Gamma^{\prime}=\left\{D_{C} \mid C \in \Gamma\right\} \cup\left\{E_{C} \mid C \in \Delta\right\} \text { for some partitions } \Gamma \text { and } \Delta \text { of } A, \\
& \text { where } D_{C}=\left\{j, c_{j}, s_{j} \mid j \in C\right\} \text { and } E_{C}=\left\{a_{j}, b_{j}, e_{j} \mid j \in C\right\} .
\end{aligned}
$$

Now consider any $i \in A$. First, note that none of $c_{i}, s_{i}, a_{i}$, and $b_{i}$ are the enemy of any other player, which is why the first part of (2) has to hold for them, i.e., $\Gamma^{\prime}(p) \succeq_{p}^{+0-1} C^{\prime} \cup\{p\}$ for $p \in\left\{c_{i}, s_{i}, a_{i}, b_{i}\right\}$ and all $C^{\prime} \in \Gamma^{\prime} \cup\{\emptyset\}$. Furthermore, for player $e_{i}$ and coalition $C^{\prime}=\emptyset$, we have $\Gamma^{\prime}\left(e_{i}\right) \succeq_{e_{i}}^{+0-\prime}\left\{e_{i}\right\}$ because there is no player in $\emptyset$ who could see $e_{i}$ as an enemy.

Since $a_{i}$ doesn't want to deviate from $\Gamma^{\prime}\left(a_{i}\right), a_{i}$ has to be together with $b_{i}$ because $b_{i}$ is $a_{i}$ 's only friend and $a_{i}$ has no enemies. Otherwise, $a_{i}$ would always prefer the coalition containing $b_{i}$. For an analogous reason, $b_{i}$ has to be together with $e_{i}$. Furthermore, $i$ cannot be in the same coalition as $e_{i}$ because $i$ is an enemy of $e_{i}$ and $e_{i}$ would rather be alone otherwise. Hence, we already know that $\left\{a_{i}, b_{i}, e_{i}\right\} \subseteq E$ and $\{i\} \subseteq D$ for some $D, E \in \Gamma^{\prime}$ with $D \neq E$. 
There remain ten cases for the allocation of $s_{i}$ and $c_{i}$. By excluding nine of these cases, it will follow that $s_{i}, c_{i} \in D$. Recall that $\Gamma^{\prime}\left(s_{i}\right) \succeq_{s_{i}}^{+0-\prime} C^{\prime} \cup\left\{s_{i}\right\}$ holds for all $C^{\prime} \in \Gamma^{\prime} \cup\{\emptyset\}$. All of the nine cases presented in the following imply that this is not true for at least one coalition $C^{\prime} \in \Gamma^{\prime} \cup\{\emptyset\}$. Hence, they cannot hold. For an overview of the cases, see Table 4.

\begin{tabular}{c|c|c|c}
\hline & $s_{i} \in E$ & $s_{i} \in D$ & $s_{i} \in F$ \\
\hline$c_{i} \in E$ & $E, D \cup\left\{s_{i}\right\}$ incomparable & $D, E \cup\left\{s_{i}\right\}$ incomparable & $F, E \cup\left\{s_{i}\right\}$ incomparable \\
$c_{i} \in D$ & $E \prec s_{i}^{+0-1} D \cup\left\{s_{i}\right\}$ & holds & $F, E \cup\left\{s_{i}\right\}$ incomparable \\
$c_{i} \in F$ & $E, D \cup\left\{s_{i}\right\}$ incomparable & $D, E \cup\left\{s_{i}\right\}$ incomparable & $F, E \cup\left\{s_{i}\right\}$ incomparable \\
$c_{i} \in G$ & - & - & $F, E \cup\left\{s_{i}\right\}$ incomparable \\
\hline
\end{tabular}

Table 4: Ten cases for the allocation of $s_{i}$ and $c_{i}$ and why nine of them cannot hold

- If $s_{i}, c_{i} \in E$ (i.e., $\left\{c_{i}, s_{i}, a_{i}, b_{i}, e_{i}\right\} \subseteq E$ and $\{i\} \subseteq D$ ), then $\Gamma^{\prime}\left(s_{i}\right)=E \nsucceq_{s_{i}}^{+0-\prime} D \cup\left\{s_{i}\right\}$. E and $D \cup\left\{s_{i}\right\}$ are incomparable with respect to $\succeq_{s_{i}}^{+0-1}$ because $E$ contains more friends but also more enemies than $D \cup\left\{s_{i}\right\}$.

- If $s_{i} \in E$ and $c_{i} \in D$ (i.e., $\left\{s_{i}, a_{i}, b_{i}, e_{i}\right\} \subseteq E$ and $\left\{i, c_{i}\right\} \subseteq D$ ), then $\Gamma^{\prime}\left(s_{i}\right)=E \prec_{s_{i}}^{+0-\prime} D \cup\left\{s_{i}\right\}$ because $D \cup\left\{s_{i}\right\}$ contains the same number of friends as $E$, but better friends than $E$, and no enemies.

- If $s_{i} \in E$ and $c_{i} \in F$ for an $F \in \Gamma^{\prime}$ with $D \neq F \neq E$ (i.e., $\left\{s_{i}, a_{i}, b_{i}, e_{i}\right\} \subseteq E,\{i\} \subseteq D$, and $\left.\left\{c_{i}\right\} \subseteq F\right)$, then $\Gamma^{\prime}\left(s_{i}\right)=E$ and $D \cup\left\{s_{i}\right\}$ are incomparable again because $E$ contains more friends but also more enemies than $D \cup\left\{s_{i}\right\}$. Hence, $\Gamma^{\prime}\left(s_{i}\right) \succeq_{s_{i}}^{+0-\prime} D \cup\left\{s_{i}\right\}$.

- If $s_{i} \in F$ for an $F \in \Gamma^{\prime}$ with $D \neq F \neq E$ (i.e., $\left\{a_{i}, b_{i}, e_{i}\right\} \subseteq E,\{i\} \subseteq D$, and $\left\{s_{i}\right\} \subseteq F$ ), then there remain four cases for $c_{i}: c_{i} \in E, c_{i} \in D, c_{i} \in F$, or $c_{i} \in G$ for a $G \in \Gamma^{\prime}$ with $G \notin\{D, E, F\}$. No matter where $c_{i}$ is, $\Gamma^{\prime}\left(s_{i}\right)=F$ and $E \cup\left\{s_{i}\right\}$ are incomparable with respect to $\succeq_{s_{i}}^{+0-\prime}$ because $E \cup\left\{s_{i}\right\}$ contains more friends but also more enemies than $F$.

- If $s_{i} \in D$ and $c_{i} \in E$ (i.e., $\left\{c_{i}, a_{i}, b_{i}, e_{i}\right\} \subseteq E$ and $\left\{i, s_{i}\right\} \subseteq D$ ), then $\Gamma^{\prime}\left(s_{i}\right)=D$ and $E \cup\left\{s_{i}\right\}$ are incomparable for $s_{i}$ because $E \cup\left\{s_{i}\right\}$ contains more friends but also more enemies than $D$.

- If $s_{i} \in D$ and $c_{i} \in F$ for an $F \in \Gamma^{\prime}$ with $D \neq F \neq E$ (i.e., $\left\{a_{i}, b_{i}, e_{i}\right\} \subseteq E,\left\{i, s_{i}\right\} \subseteq D$, and $\left.\left\{c_{i}\right\} \subseteq F\right)$, then $\Gamma^{\prime}\left(s_{i}\right)=D$ and $E \cup\left\{s_{i}\right\}$ again are incomparable for $s_{i}$.

The only remaining case is $s_{i}, c_{i} \in D$ (i.e., $\left\{a_{i}, b_{i}, e_{i}\right\} \subseteq E$ and $\left\{i, c_{i}, s_{i}\right\} \subseteq D$ ). Note that this case indeed fulfills $\Gamma^{\prime}\left(s_{i}\right) \succeq_{s_{i}}^{+0-\prime} C^{\prime} \cup\left\{s_{i}\right\}$ for all $C^{\prime} \in \Gamma^{\prime} \cup\{\emptyset\}$. Hence, for every $i \in A$, we have $\left\{a_{i}, b_{i}, e_{i}\right\} \subseteq E_{i}$ and $\left\{i, c_{i}, s_{i}\right\} \subseteq D_{i}$ for some $D_{i}, E_{i} \in \Gamma^{\prime}$ with $D_{i} \neq E_{i}$. It furthermore holds for any $i, j \in A$ that $E_{i} \neq D_{j}$. Otherwise, we had $E_{i}=D_{j} \supseteq\left\{a_{i}, b_{i}, e_{i}, j, c_{j}, s_{j}\right\}$. Since $j$ is an enemy of $e_{i}, e_{i}$ would like to deviate to the empty coalition which is a contradiction to the assumption, see Equation (2). It follows that $\Gamma^{\prime}$ has the form presented above.

Finally, consider the clone players $c_{i} \in$ Clone. Equation (2) also holds for $c_{i}$, i.e., we have

$$
\left(\forall C^{\prime} \in \Gamma^{\prime} \cup\{\emptyset\}\right)\left[\Gamma^{\prime}\left(c_{i}\right) \succeq_{c_{i}}^{+0-\prime} C^{\prime} \cup\left\{c_{i}\right\} \vee\left(\exists x \in C^{\prime}\right)\left[c_{i} \in A_{x}^{-\prime}\right]\right]
$$


Since $c_{i}$ is not the enemy of any other player, i.e., $c_{i} \notin A_{x}^{-\prime}$ for all $x \in A^{\prime}$, it follows that

$$
\left(\forall C^{\prime} \in \Gamma^{\prime} \cup\{\emptyset\}\right)\left[\Gamma^{\prime}\left(c_{i}\right) \succeq_{c_{i}}^{+0-\prime} C^{\prime} \cup\left\{c_{i}\right\}\right] .
$$

Recall that $\Gamma^{\prime}=\left\{D_{C} \mid C \in \Gamma\right\} \cup\left\{E_{C} \mid C \in \Delta\right\}$ for some partitions $\Gamma$ and $\Delta$ of $A$ with $D_{C}=\left\{j, c_{j}, s_{j} \mid j \in\right.$ $C\}$ and $E_{C}=\left\{a_{j}, b_{j}, e_{j} \mid j \in C\right\}$. Furthermore, note that $\Gamma^{\prime}\left(c_{i}\right)=D_{\Gamma(i)}$ and let $D_{\emptyset}=\emptyset$. Equation (3) in particular holds for all $C^{\prime}=D_{C} \in \Gamma^{\prime} \cup\{\emptyset\}$ with $C \in \Gamma \cup\{\emptyset\}$. Hence, we have

$$
(\forall C \in \Gamma \cup\{\emptyset\})\left[D_{\Gamma(i)} \succeq_{c_{i}}^{+0-\prime} D_{C} \cup\left\{c_{i}\right\}\right]
$$

Because $c_{i}$ is neutral to all players $x \in A^{\prime}$ with $x \notin A, x \neq c_{i}$, we can remove all these players from (4). With $D_{C} \cap A=C$, we get $(\forall C \in \Gamma \cup\{\emptyset\})\left[\Gamma(i) \cup\left\{c_{i}\right\} \succeq_{c_{i}}^{+0-\prime} C \cup\left\{c_{i}\right\}\right] . c_{i}$ is also neutral to $i$. Hence, we can remove $i$ on the left-hand side and get

$$
(\forall C \in \Gamma \cup\{\emptyset\})\left[\Gamma(i) \backslash\{i\} \cup\left\{c_{i}\right\} \succeq_{c_{i}}^{+0-\prime} C \cup\left\{c_{i}\right\}\right] .
$$

Finally, $c_{i}$ has the same friends, order over friends, enemies, and order over enemies as player $i$ has in $H$. Therefore, (5) is equivalent to $(\forall C \in \Gamma \cup\{\emptyset\})\left[\Gamma(i) \succeq_{i}^{+0-} C \cup\{i\}\right]$. Thus the coalition structure $\Gamma$ is necessarily Nash stable for $H$ and $H \in$ NNSE.

\subsection{Core Stability and Strict Core Stability}

We now turn to group deviations and start with possible (strict) core stability. We first state some characterizations, which show that possible and necessary verification are in coNP for both core stability and strict core stability.

Proposition $49 \Gamma$ is not possibly core stable if and only if there is a coalition $C \subseteq A, C \neq \emptyset$ such that $\Gamma(i) \prec_{i}^{+\Gamma(i)} C$ holds for all $i \in C$.

Proof. From left to right, assume that $\Gamma$ is not possibly core stable, i.e., that for every profile $\left(\succeq_{1}, \ldots, \succeq_{n}\right) \in \times_{i=1}^{n} \operatorname{Ext}\left(\succeq_{i}^{+0-}\right)$ we have a blocking coalition $C \subseteq A, C \neq \emptyset$. Consider some profile $\left(\succeq_{1}, \ldots, \succeq_{n}\right) \in \times_{i=1}^{n} \operatorname{Ext}\left(\succeq_{i}^{+\Gamma(i)}\right)$. This extended profile does exist since $\succ_{i}^{+\Gamma(i)}$ is acyclic for all $i \in A$ by Proposition 16. It then holds for every $i \in A$ that $\succeq_{i}$ extends $\succeq_{i}^{+\Gamma(i)}$ and that $\succeq_{i}^{+\Gamma(i)}$ extends $\succeq_{i}^{+0-}$ by Proposition 17. Hence, $\succeq_{i}$ also extends $\succeq_{i}^{+0-}$. Then, there also is a blocking coalition $C \subseteq A, C \neq \emptyset$ for this profile, i.e., $\Gamma(i) \prec_{i} C$ for every $i \in C$. Since $\succeq_{i}$ extends $\succeq_{i}^{+\Gamma(i)}$ and with Observation 18 it follows that $\Gamma(i) \prec_{i}^{+\Gamma(i)} C$ for every $i \in C$.

From right to left, assume that there is a coalition $C \subseteq A, C \neq \emptyset$ with $\Gamma(i) \prec_{i}^{+\Gamma(i)} C$ for every $i \in C$. By Proposition 19.2(d), it follows for each $i \in C$ that all extensions $\succeq_{i} \in \operatorname{Ext}\left(\succeq_{i}^{+0-}\right)$ satisfy $C \succ_{i} \Gamma(i)$. Hence, $C$ blocks $\Gamma$ for every extended profile and $\Gamma$ is not possibly core stable.

Proposition $50 \Gamma$ is not necessarily core stable if and only if there is a coalition $C \subseteq A, C \neq \emptyset$ such that $\Gamma(i) \prec_{i}^{-\Gamma(i)} C$ holds for all $i \in C$. 
Proof. From left to right, assume that $\Gamma$ is not necessarily core stable. Then there exists a profile $\left(\succeq_{1}, \ldots, \succeq_{n}\right) \in \times_{i=1}^{n} \operatorname{Ext}\left(\succeq_{i}^{+0-}\right)$ and a coalition $C \subseteq A, C \neq \emptyset$ such that $\Gamma(i) \prec_{i} C$ holds for all $i \in C$. With Proposition 19.1(d), it follows that $\Gamma(i) \prec_{i}^{-\Gamma(i)} C$ for all $i \in C$.

From right to left, assume that there is a coalition $C \subseteq A, C \neq \emptyset$ with $\Gamma(i) \prec_{i}^{-\Gamma(i)} C$ holds for all $i \in C$. Again, by Proposition 19.1(d), it follows for all $i \in C$ that there is an extension $\succeq_{i} \in$ $\operatorname{Ext}\left(\succeq_{i}^{+0-}\right)$ such that $\Gamma(i) \prec_{i} C$. Hence, $\Gamma$ is not necessarily core stable.

Proposition $51 \Gamma$ is not possibly strictly core stable if and only if there is a nonempty coalition $C \subseteq A$ such that $\Gamma(i) \preceq_{i}^{+\Gamma(i)} C$ for each player $i \in C$, and $\Gamma(j) \prec_{j}^{+\Gamma(j)}$ C for some player $j \in C$.

Proof. This can be shown similarly to Proposition 49, using Propositions 19.2(c) and 19.2(d).

Proposition $52 \Gamma$ is not necessarily strictly core stable if and only if there is a nonempty coalition $C \subseteq A$ such that $\Gamma(i) \preceq_{i}^{-\Gamma(i)} C$ for each player $i \in C$, and $\Gamma(j) \prec_{j}^{-\Gamma(j)}$ C for some player $j \in C$.

Proof. This can be shown similarly to Proposition 50, using Propositions 19.1(c) and 19.1(d).

Since the relations $\succeq_{i}^{+C}$ and $\succeq_{i}^{-C}$ can be decided in polynomial time for any two coalitions, we can choose a coalition structure $\Delta$ nondeterministically and verify the characterizations from Propositions 49, 50, 51, and 52 in polynomial time. Therefore, we get the following corollary.

Corollary 53 Possible-Core-Stability-VerificAtion, NeCESSARY-CORE-STABILityVerification, Possible-Strict-CORE-Stability-Verification, and NeCESSARY-STRICTCORE-STABILITY-VERIFICATION are in coNP.

Theorem 54 The problems Possible-Core-Stability-VERIFICATION and Possible-STRICTCORE-STABILITY-VERIFICATION are coNP-complete.

Proof. The coNP upper bounds are already given by Corollary 53. Hardness for coNP of both problems can be shown by means of the reduction from CLIQUE to the complement of the core stability verification problem in the enemy-oriented representation (Sung \& Dimitrov, 2007). Note that this representation is a special case of the representation with ordinal preferences and thresholds where there are no neutral players and only indifferences between all friends and between all enemies in a player's preference. Furthermore, note that the enemy-oriented preference extension is contained in $\times_{i=1}^{n} \operatorname{Ext}\left(\succeq_{i}^{+0-}\right)$. While a "clique" of friends is necessarily preferred by all its members to a coalition containing fewer friends or even more enemies, there does not necessarily exist a blocking coalition if there is no such clique (for example, there is no blocking coalition in the enemy-oriented preference extension). 


\subsection{Popularity and Strict Popularity}

We now consider the different stability problems regarding (strict) popularity. We first state characterizations for possible and necessary (strict) popularity, using the optimistic and pessimistic extensions from Definition 10.

Proposition $55 \Gamma$ is not possibly popular if and only if there is a coalition structure $\Delta$ such that the number of players $i \in A$ with $\Gamma(i) \succ_{i}^{+\Gamma(i)} \Delta(i)$ is smaller than the number of players $j \in A$ with $\Delta(j) \succ_{j}^{+\Gamma(j)} \Gamma(j)$.

Proof. From left to right, assume that $\Gamma$ is not possibly popular, i.e., for every $\left(\succeq_{1}, \ldots, \succeq_{n}\right) \in$ $\times_{i=1}^{n} \operatorname{Ext}\left(\succeq_{i}^{+0-}\right)$ there is a coalition structure $\Delta \neq \Gamma$ such that the number of players $i \in A$ with $\Gamma(i) \succ_{i} \Delta(i)$ is smaller than the number of players $j \in A$ with $\Delta(j) \succ_{j} \Gamma(j)$. Consider any profile $\left(\succeq_{1}, \ldots, \succeq_{n}\right) \in \times_{i=1}^{n} \operatorname{Ext}\left(\succeq_{i}^{+\Gamma(i)}\right)$, which certainly exists due to Proposition 16 . Since $\left(\succeq_{1}, \ldots, \succeq_{n}\right)$ $\in \times_{i=1}^{n} \operatorname{Ext}\left(\succeq_{i}^{+0-}\right)$, the above inequality also holds for this profile.

For all $i \in A$ with $\Gamma(i) \nsucc_{i} \Delta(i)$, it holds that $\Gamma(i) \nsucc_{i}^{+\Gamma(i)} \Delta(i)$ because $\succeq_{i}$ is an extension of $\succeq_{i}^{+\Gamma(i)}$. Hence, $\left|\left\{i \in A \mid \Gamma(i) \succ_{i}^{+\Gamma(i)} \Delta(i)\right\}\right| \leq\left|\left\{i \in A \mid \Gamma(i) \succ_{i} \Delta(i)\right\}\right|$. Furthermore, consider all $j \in A$ with $\Delta(j) \succ_{j} \Gamma(j)$. With Observation 18 and because $\succeq_{j}$ extends $\succeq_{j}^{+\Gamma(i)}$ it follows that $\Delta(j) \succ_{j}^{+\Gamma(i)} \Gamma(j)$. Hence, $\left|\left\{j \in A \mid \Delta(j) \succ_{j} \Gamma(j)\right\}\right| \leq\left|\left\{j \in A \mid \Delta(j) \succ_{j}^{+\Gamma(i)} \Gamma(j)\right\}\right|$. Combining all inequalities, we get $\left|\left\{i \in A \mid \Gamma(i) \succ_{i}^{+\Gamma(i)} \Delta(i)\right\}\right| \leq\left|\left\{i \in A \mid \Gamma(i) \succ_{i} \Delta(i)\right\}\right|<\left|\left\{j \in A \mid \Delta(j) \succ_{j} \Gamma(j)\right\}\right| \leq \mid\left\{j \in A \mid \Delta(j) \succ_{j}^{+\Gamma(j)}\right.$ $\Gamma(j)\} \mid$.

From right to left, assume that there is a coalition structure $\Delta \neq \Gamma$ such that the number of players $i \in A$ with $\Gamma(i) \succ_{i}^{+\Gamma(i)} \Delta(i)$ is smaller than the number of players $j \in A$ with $\Delta(j) \succ_{j}^{+\Gamma(j)} \Gamma(j)$.

Consider all $i \in A$ with $\Gamma(i) \nsucc_{i}^{+\Gamma(i)} \Delta(i)$. It follows by Observation 18 that $\Delta(i) \succeq_{i}^{+\Gamma(i)} \Gamma(i)$ which, by Proposition 14, implies $\Delta(i) \succeq_{i}^{+0-} \Gamma(i)$. Hence, $\Gamma(i) \nsucc_{i} \Delta(i)$ for any extension $\succeq_{i}$ of $\succeq_{i}^{+0-}$. It follows that $\left|\left\{i \in A \mid \Gamma(i) \succ_{i} \Delta(i)\right\}\right| \leq\left|\left\{i \in A \mid \Gamma(i) \succ_{i}^{+\Gamma(i)} \Delta(i)\right\}\right|$ for all $\succeq_{i} \in \operatorname{Ext}\left(\succeq_{i}^{+0-}\right)$. Furthermore, consider all $j \in A$ with $\Delta(j) \succ_{j}^{+\Gamma(j)} \Gamma(j)$. It follows by Proposition 14 that $\Delta(j) \succ_{j}^{+0-}$ $\Gamma(j)$. Hence, for any extension $\succeq_{i}$ of $\succeq_{i}^{+0-}$, it holds that $\Delta(j) \succ_{j} \Gamma(j)$ and $\mid\left\{j \in A \mid \Delta(j) \succ_{j}^{+\Gamma(j)}\right.$ $\Gamma(j)\}|\leq|\left\{j \in A \mid \Delta(j) \succ_{j} \Gamma(j)\right\} \mid$. Summing up all inequalities, $\left|\left\{i \in A \mid \Gamma(i) \succ_{i} \Delta(i)\right\}\right| \leq \mid\{i \in$ $\left.A \mid \Gamma(i) \succ_{i}^{+\Gamma(i)} \Delta(i)\right\}|<|\left\{j \in A \mid \Delta(j) \succ_{j}^{+\Gamma(j)} \Gamma(j)\right\}|\leq|\left\{j \in A \mid \Delta(j) \succ_{j} \Gamma(j)\right\} \mid$ holds for all extensions $\succeq_{i}$ of $\succeq_{i}^{+0-}$ and $\Gamma$ is not possibly popular.

Proposition $56 \Gamma$ is not necessarily popular if and only if there is a coalition structure $\Delta$ such that the number of players $i \in A$ with $\Gamma(i) \succ_{i}^{-\Gamma(i)} \Delta(i)$ is smaller than the number of players $j \in A$ with $\Delta(j) \succ_{j}^{-\Gamma(j)} \Gamma(j)$.

Proof. From left ro right, assume that $\Gamma$ is not necessarily popular. It then holds that there is a $\left(\succeq_{1}, \ldots, \succeq_{n}\right) \in \times_{i=1}^{n} \operatorname{Ext}\left(\succeq_{i}^{+0-}\right)$ and a coalition structure $\Delta \neq \Gamma$ such that the number of players $i \in A$ with $\Gamma(i) \succ_{i} \Delta(i)$ is smaller than the number of players $j \in A$ with $\Delta(j) \succ_{j} \Gamma(j)$.

By the definition of extensions (Definition 8), it holds for every $i \in A$ with $\Gamma(i) \nsucc_{i} \Delta(i)$ that $\Gamma(i) \nsucc_{i}^{+0-} \Delta(i)$. This, by Proposition 14, implies $\Delta(i) \succeq_{i}^{-\Gamma(i)} \Gamma(i)$. Hence, $\Gamma(i) \nsucc_{i}^{-\Gamma(i)} \Delta(i)$ for every $i \in A$ with $\Gamma(i) \nsucc_{i} \Delta(i)$. This means that the number of players $i \in A$ with $\Gamma(i) \succ_{i}^{-\Gamma(i)} \Delta(i)$ is less 
than or equal to the number of players $i \in A$ with $\Gamma(i) \succ_{i} \Delta(i)$. Furthermore, by the definition of extensions, it holds for every $j \in A$ with $\Delta(j) \succ_{j} \Gamma(j)$ that $\Gamma(j) \succeq_{j}^{+0-} \Delta(j)$. With Proposition 14, this implies $\Gamma(j) \nsucceq_{j}^{-\Gamma(j)} \Delta(j)$. Since $\succeq_{j}^{-\Gamma(j)}$ is never undecided concerning $\Gamma(j)$ by Observation 18 , we have $\Delta(j) \succ_{j}^{-\Gamma(j)} \Gamma(j)$. Hence, the number of players $j \in A$ with $\Delta(j) \succ_{j}^{-\Gamma(j)} \Gamma(j)$ is at least as large as the number of players $j \in A$ with $\Delta(j) \succ_{j} \Gamma(j)$. Combining all inequalities, we get $\left|\left\{i \in A \mid \Gamma(i) \succ_{i}^{-\Gamma(i)} \Delta(i)\right\}\right| \leq\left|\left\{i \in A \mid \Gamma(i) \succ_{i} \Delta(i)\right\}\right|<\left|\left\{j \in A \mid \Delta(j) \succ_{j} \Gamma(j)\right\}\right| \leq \mid\left\{j \in A \mid \Delta(j) \succ_{j}^{-\Gamma(j)}\right.$ $\Gamma(j)\} \mid$.

From right to left, assume that there is a coalition structure $\Delta \neq \Gamma$ such that $\mid\left\{i \in A \mid \Gamma(i) \succ_{i}^{-\Gamma(i)}\right.$ $\Delta(i)\}|<|\left\{j \in A \mid \Delta(j) \succ_{j}^{-\Gamma(j)} \Gamma(j)\right\} \mid$. Consider a profile $\left(\succeq_{1}, \ldots, \succeq_{n}\right) \in \times_{i=1}^{n} \operatorname{Ext}\left(\succeq_{i}^{-\Gamma(i)}\right)$ of complete extensions, which exists since $\succ_{i}^{-\Gamma(i)}$ is acyclic (Proposition 16). Note that each $\succeq_{i}$ is also an extension of $\succeq_{i}^{+0-}$ due to Proposition 17.

By Observation 18, it holds for every $i \in A$ with $\Gamma(i) \nsucc_{i}^{-\Gamma(i)} \Delta(i)$ that $\Gamma(i) \preceq_{i}^{-\Gamma(i)} \Delta(i)$. Hence, it holds for every extension $\succeq_{i}$ of $\succeq_{i}^{-\Gamma(i)}$ that $\Gamma(i) \preceq_{i} \Delta(i)$. This implies $\Gamma(i) \nsucc_{i} \Delta(i)$. Hence, $\mid\{i \in$ $\left.A \mid \Gamma(i) \succ_{i} \Delta(i)\right\}|\leq|\left\{i \in A \mid \Gamma(i) \succ_{i}^{-\Gamma(i)} \Delta(i)\right\} \mid$. Furthermore, for every $j \in A$ with $\Delta(j) \succ_{j}^{-\Gamma(j)} \Gamma(j)$ it holds that $\Delta(j) \succ_{j} \Gamma(j)$ because $\succeq_{j}$ extends $\succeq_{j}^{-\Gamma(j)}$. Hence, $\left|\left\{j \in A \mid \Delta(j) \succ_{j}^{-\Gamma(j)} \Gamma(j)\right\}\right| \leq \mid\{j \in$ $\left.A \mid \Delta(j) \succ_{j} \Gamma(j)\right\} \mid$. Summing up, it holds for this extended profile that $\left|\left\{i \in A \mid \Gamma(i) \succ_{i} \Delta(i)\right\}\right| \leq \mid\{i \in$ $\left.A \mid \Gamma(i) \succ_{i}^{-\Gamma(i)} \Delta(i)\right\}|<|\left\{j \in A \mid \Delta(j) \succ_{j}^{-\Gamma(j)} \Gamma(j)\right\}|\leq|\left\{j \in A \mid \Delta(j) \succ_{j} \Gamma(j)\right\} \mid$, which means that $\Gamma$ is not necessarily popular.

The proofs of the following two propositions are similar to those of Propositions 55 and 56 and are therefore omitted.

Proposition $57 \Gamma$ is not possibly strictly popular if and only if there is a coalition structure $\Delta$ such that the number of players $i \in A$ with $\Gamma(i) \succ_{i}^{+\Gamma(i)} \Delta(i)$ is smaller than or equal to the number of players $j \in A$ with $\Delta(j) \succ_{j}^{+\Gamma(j)} \Gamma(j)$.

Proposition $58 \Gamma$ is not necessarily popular if and only if there is a coalition structure $\Delta$ such that the number of players $i \in A$ with $\Gamma(i) \succ_{i}^{-\Gamma(i)} \Delta(i)$ is smaller than or equal to the number of players $j \in A$ with $\Delta(j) \succ_{j}^{-\Gamma(j)} \Gamma(j)$.

Since a coalition structure $\Delta$ can be chosen nondeterministically, and it can be verified in polynomial time whether the inequalities in Propositions 55, 56, 57, and 58 hold, we get the following corollary.

Corollary 59 Possible-Popularity-Verification, Necessary-Popularity-VerificaTION, Possible-STRICT-Popularity-VERIFICATION, and NECESSARY-STRICT-POPUlarityVERIFICATION are in cONP.

To show NP-hardness of the stability problems regarding (strict) popularity, we can make use of several games constructed from a given $\mathrm{X} 3 \mathrm{C}$ instance, which we state in the following construction that is inspired by the proof of Theorem 3 of Sung and Dimitrov (2010) and is also based on ideas in the proof of Theorem 43.

Construction 60 Let $(B, \mathscr{S})$ be an $\mathrm{X} 3 \mathrm{C}$ instance. All four games have three types of players: connection players, element players, and set players. 
1. Let $A=\left\{\alpha_{b, i} \mid b \in B, 1 \leq i \leq 3 m+3\right\} \cup\left\{\beta_{b} \mid b \in B\right\} \cup \bigcup_{S \in \mathscr{S}} Q_{S}$ be the set of players and define the weak rankings with double threshold as follows:

Connection Players: For each $b \in B$, we have $3 m+3$ connection players $\alpha_{b, i}$ for $i, 1 \leq$ $i \leq 3 m+3$. These players consider all remaining connection players corresponding to the same element $b \in B$ to be friends. The first $3 m$ of these players also consider their corresponding element player $\beta_{b}$ to be a friend and even rank her on the first position. Formally, for each $b \in B$, and for each $i, 2 \leq i \leq 3 m+3$,

$$
\begin{aligned}
& \unrhd_{\alpha_{b, 1}}^{+0-}=\left(\beta_{b} \sim\left\{\alpha_{b, j} \mid j \neq 1\right\}_{\sim}|\emptyset|\{\text { other players }\}_{\sim}\right), \\
& \unrhd_{\alpha_{b, i}}^{+0-}=\left(\left\{\alpha_{b, j} \mid j \neq i\right\}_{\sim}\left|\left\{\beta_{b}\right\}\right|\{\text { other players }\}_{\sim}\right) .
\end{aligned}
$$

Element players: For each $b \in B$, there is one element player $\beta_{b}$. Each of these players considers all set players corresponding to sets containing $b$ to be her best friends, followed by the remaining element players. The least preferred friends are all connection players corresponding to the specific element $b$, which are collected in the set $C_{b}$. As there are no neutral players, all other players are considered to be enemies. Formally, for each $b \in B$, define $C_{b}=\left\{\alpha_{b, i} \mid 1 \leq i \leq 3 m+3\right\}$ and

$$
\unrhd_{\beta_{b}}^{+0-}=\left(\bigcup_{\{S \mid b \in S\}} Q_{S \sim} \triangleright\left\{\beta_{b^{\prime}} \mid b^{\prime} \neq b\right\}_{\sim} \triangleright C_{b \sim}|\emptyset|\{\text { other players }\}_{\sim}\right) .
$$

Set players: For each $S \in \mathscr{S}$, we have $3 m+1$ set players in the set $Q_{S}=\left\{\zeta_{S, k} \mid 1 \leq k \leq\right.$ $3 m+1\}$. For each fixed $S \in \mathscr{S}$, these players only consider the set players in $Q_{S}$ to be their friends and have all element players corresponding to the elements in $S$ in their set of neutral players. All remaining players are their enemies. Formally, for each $S \in \mathscr{S}$ and for each $k, 1 \leq k \leq 3 m+1$,

$$
\unrhd_{\zeta_{S, k}}^{+0-}=\left(\left\{\zeta_{S, k^{\prime}} \mid k^{\prime} \neq k\right\}_{\sim}\left|\left\{\beta_{b} \mid b \in S\right\}\right|\{\text { other players }\}_{\sim}\right)
$$

The profile is illustrated in Figure 7.

2. In the second game, we have the same set of players as in the first game (given in Construction 60.1), namely $A=\left\{\alpha_{b, i} \mid b \in B, 1 \leq i \leq 3 m+3\right\} \cup\left\{\beta_{b} \mid b \in B\right\} \cup \cup_{S \in \mathscr{S}} Q_{S}$. The weak rankings with double threshold also are the same, except for the first connection player corresponding to the last element in $B: \unrhd_{\alpha_{3 m, 1}}^{+0-}=\left(\left\{\alpha_{3 m, j} \mid j \neq 1\right\}_{\sim}\left|\left\{\beta_{3 m}\right\}\right|\{\text { other players }\}_{\sim}\right)$.

3. The third game is another modification of the first game (presented in Construction 60.1). Here, for each $b \in B$, there are only $3 m+2$ connection players $\alpha_{b, i}$. Thus the set of players is given by $A=\left\{\alpha_{b, i} \mid b \in B, 1 \leq i \leq 3 m+2\right\} \cup\left\{\beta_{b} \mid b \in B\right\} \cup \bigcup_{S \in \mathscr{S}} Q_{S}$.

The preferences of the set players remain the same while those for the element players are changed to $\unrhd_{\beta_{b}}^{+0-}=\left(C_{b \sim} \triangleright \bigcup_{\{S \mid b \in S\}} Q_{S \sim} \triangleright\left\{\beta_{b^{\prime}} \mid b^{\prime} \neq b\right\}_{\sim}|\emptyset|\{\text { other players }\}_{\sim}\right)$.

4. The fourth game is a modification of the third game (presented in Construction 60.3). The set of players and their weak rankings with double threshold remain the same, except for the 


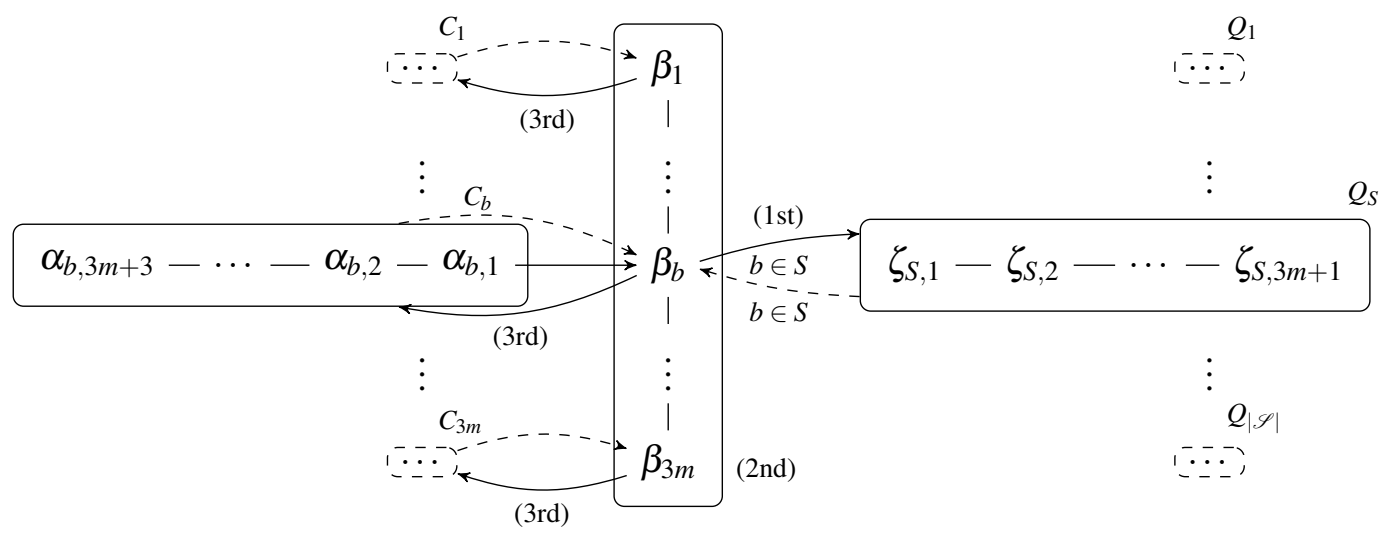

Figure 7: Network of friends from Construction 60.1 that is used in the proof of Theorem 61

preference of the first connection player corresponding to the last element in $B$, namely $\alpha_{3 m, 1}$. In this game, she considers the corresponding element player $\beta_{3 m}$ to be a neutral player and not a friend: $\unrhd_{\alpha_{3 m, 1}^{+0-}}^{+0}=\left(\left\{\alpha_{3 m, j} \mid j \neq 1\right\}_{\sim}\left|\left\{\beta_{3 m}\right\}\right|\{\text { other players }\}_{\sim}\right)$.

We summarize our results for verifying possibly and necessary (strictly) popular coalition structures in the following theorem.

Theorem 61 Possible-STRICT-Popularity-Verification, Possible-Popularity-VerifiCATION, NeCESSARy-STRICT-Popularity-VerifiCATIOn, and NeCESSARy-PopularityVERIFICATION are coNP-complete.

Proof. We start with the first part of the theorem, namely the proof of coNP-hardness of POSSIBLESTRICT-Popularity-Verification. Consider the game from Construction 60.1 and let

$$
\Gamma=\left\{C_{b} \cup\left\{\beta_{b}\right\} \mid b \in B\right\} \cup\left\{Q_{S} \mid S \in \mathscr{S}\right\}
$$

be the coalition structure of interest. We show that $\Gamma$ is possibly strictly popular if and only if there is no solution for $(B, \mathscr{S})$.

Only if: Assuming $(B, \mathscr{S})$ has a solution $\mathscr{S}^{\prime}$, we consider the coalition structure

$$
\Gamma^{\prime}=\left\{C_{b} \mid b \in B\right\} \cup\left\{P_{S} \mid S \in \mathscr{S}^{\prime}\right\} \cup\left\{Q_{S} \mid S \notin \mathscr{S}^{\prime}\right\} .
$$

There are $3 m$ players, namely $\alpha_{b, 1}$ for each $b \in B$, who necessarily prefer $\Gamma\left(\alpha_{b, 1}\right)$ to $\Gamma^{\prime}\left(\alpha_{b, 1}\right)$, and $3 m$ players, namely all element players $\beta_{b}$ for $b \in B$, who necessarily prefer $\Gamma^{\prime}\left(\beta_{b}\right)$ to $\Gamma\left(\beta_{b}\right)$. All other players are indifferent between their coalitions in $\Gamma$ and $\Gamma^{\prime}$. Thus $\Gamma$ is necessarily prevented from being strictly popular.

If: Assume now that $\Gamma$ is not possibly strictly popular, that is, for each preference extension, there exists another coalition structure $\Gamma^{\prime}$ that beats $\Gamma$ in pairwise comparison. All players $\alpha_{b, i}$ with $b \in B$ and $1 \leq i \leq 3 m+3$, and all players $\zeta_{S, k}$ with $S \in \mathscr{S}$ and $1 \leq k \leq 3 m+1$, are in one of their favorite coalitions in $\Gamma$; hence, they cannot improve in $\Gamma^{\prime}$. Therefore, there are at most $3 m$ players (which have to be element players $\beta_{b}$ with $b \in B$ ) who vote in favor of $\Gamma^{\prime}$. 
If, for some $b \in B$, not all players $\alpha_{b, i}, 1 \leq i \leq 3 m+3$, are together, they are all worse off in comparison to $\Gamma$. This cannot be counterbalanced by the $3 m$ element players; consequently, they have to be in one coalition in $\Gamma^{\prime}$. For the same reason, for each $S \in \mathscr{S}$, the $3 m+1$ players in $Q_{S}$ cannot be separated in $\Gamma^{\prime}$.

If some element player $\beta_{b}$ with $b \in B$ wants to improve by adding friends to $C_{b} \cup\left\{\beta_{b}\right\}$, all $3 m+3$ players in $C_{b}$ will disapprove; hence, this cannot be the case in $\Gamma^{\prime}$ either. Thus, for each $b \in B$, the player $\alpha_{b, 1}$ is separated from $\beta_{b}$, which sums up in a number of $3 m$ players in favor of $\Gamma$ in comparison to $\Gamma^{\prime}$. This means that, in order for $\Gamma^{\prime}$ to be successful, each $\beta_{b}$ has to prefer $\Gamma^{\prime}\left(\beta_{b}\right)$ to $\Gamma\left(\beta_{b}\right)$. It necessarily holds that an element player $\beta_{b}$ has the following preferences $Q_{S} \cup$ $\left\{\beta_{b}, \beta_{b^{\prime}}\right\} \succ_{\beta_{b}} Q_{S} \cup\left\{\beta_{b} \mid b \in S\right\} \succ_{\beta_{b}}\left\{\beta_{b^{\prime}} \mid b \in B\right\}$, where $\beta_{b^{\prime}}$ is another element player corresponding to an element from the same $S \in \mathscr{S}$ (i.e., $b \neq b^{\prime}$ and $b, b^{\prime} \in S$ ); it furthermore necessarily holds that $C_{b} \cup\left\{\beta_{b}\right\} \sim_{\beta_{b}} Q_{S} \cup\left\{\beta_{b}, \beta_{b^{\prime}}\right\}$. However, there exists a preference extension in which this indifference is solved in favor of $\Gamma\left(\beta_{b}\right)$. Thus, for this preference extension, $Q_{S} \cup\left\{\beta_{b}, \beta_{b^{\prime}}\right\}$ cannot be in $\Gamma$ (nor can any coalition even less preferred by $\beta_{b}$ be in $\Gamma$ ). Also, there cannot be any enemies of players $Q_{S}$ in the same coalition, since otherwise there would be at least $3 m+1$ more players that disapprove. This leaves only one possibility: There is a coalition structure such that all players $\beta_{r}$ are in a coalition $P_{S}$ with $b \in S$. Consequently, there is an exact cover of $B$ by sets in $\mathscr{S}$. Thus PossibleSTRICT-POPULARITY-VERIFICATION is coNP-hard.

For Possible-Popularity-Verification, consider the game given in Construction 60.2 and the two coalition structures, $\Gamma$ and $\Gamma^{\prime}$, defined in Equations (6) and (7).

Note that now we need $\Gamma^{\prime}$ to strictly defeat $\Gamma$, in order to obtain that $\Gamma$ is not possibly popular. The argumentation is analogous to above, except that now only $3 m-1$ players of the form $\alpha_{b, 1}$, $b \in B$, dislike being in a different coalition than $\beta_{b}$. Then, for each preference extension, there is such a $\Gamma^{\prime}$ if and only if all element players $\beta_{b}, b \in B$, can be placed in some $P_{S}, b \in S$.

Thus it holds that $\Gamma$ is possibly popular if and only if there is no solution for $(B, \mathscr{S})$.

For the coNP-hardness proof of NECESSARY-STRICT-POPULARITY-VERIFICATION, we consider the game given in Construction 60.3. In this game it holds that possibly $P_{S} \succ_{\beta_{b}} C_{b} \cup\left\{\beta_{b}\right\}$ and necessarily $C_{b} \cup\left\{\beta_{b}\right\} \succ_{\beta_{b}} Q_{S} \cup R_{S} \succ_{\beta_{b}}\left\{\beta_{b^{\prime}} \mid b \in B\right\}$, for each $b \in B$, where $R_{S} \subseteq\left\{\beta_{b} \mid b \in S\right\}$. Similarly to the previous argumentation, it can be shown that the coalition structure $\Gamma$ defined in Equation (6) is necessarily strictly popular if and only if there is no solution for $(B, \mathscr{S})$.

For the last problem, namely NECESSARY-POPULARITY-VERIFICATION, we can show coNPhardness via a reduction from $\mathrm{X} 3 \mathrm{C}$ given by the game defined in Construction 60.4. With a similar argumentation as above, we have that the coalition structure $\Gamma$ defined in Equation (6) is necessarily popular if and only if there is no solution for $(B, \mathscr{S})$.

Finally, the coNP upper bounds hold by Corollary 59.

For strict popularity, both existence problems are coNP-hard.

Theorem 62 Possible-Strict-Popularity-Existence and Necess ary-Strict-PopularITY-EXISTENCE are coNP-hard.

Proof. To show coNP-hardness of Possible-Strict-Popularity-Existence, we consider the game defined in Construction 60.1. We have seen in the proof of Theorem 61 that if there is no solution for the given $\mathrm{X} 3 \mathrm{C}$ instance there exists a possibly strictly popular coalition structure (namely $\Gamma$ defined in Equation (6)). Now we show that, if there is a solution for the given X3C instance, not only is $\Gamma$ beaten in pairwise comparison, but there is no other strictly popular coalition 
structure either. Observe that $\Gamma$ and $\Gamma^{\prime}$ (the latter is defined in Equation (7)) tie up in pairwise comparison with the maximal number of positive votes each $(3 \mathrm{~m})$. Thus these two cannot be strictly popular. Any other coalition structure can also only possibly gain at most $3 m$ positive votes; hence, there is no coalition structure that beats every other coalition structure in pairwise comparison.

For the coNP-hardness proof of NeCESSARY-STRICT-PopUlARITY-ExisTENCE, we can use the game defined in Construction 60.3. We have seen that in this game the coalition structure $\Gamma$ defined in Equation (6) is necessarily strictly popular, so there exists a necessarily strictly popular coalition structure. By analogous arguments as above, while all coalition structures other than $\Gamma$ and $\Gamma^{\prime}$ (where, again, the latter is defined in Equation (7)) are even necessarily worse off, it can be seen that if there is a solution for the original X3C instance, there is no necessarily strictly popular coalition structure at all.

\section{Conclusions and Future Work}

We have introduced a new representation of preferences in hedonic games using the polarized responsive principle to extend the players' preferences over the other players to preferences over coalitions. Generalizing the responsive extension principle to neutral items in addition to positive and negative items (here called friends and enemies) is novel and original in itself, independently of its use in hedonic games. That is, the polarized extension can be useful more generally in all contexts where some agents may have positive or negative preferences for the presence of some entity. Two important examples are multiwinner elections and fair division.

Regarding multiwinner elections (see, e.g., Faliszewski, Skowron, Slinko, \& Talmon, 2017), beyond ranking candidates, it makes sense for voters to specify, for any of the candidates, whether they would prefer to have them in the committee or not; and for some of the candidates voters may not care about whether they are in the committee or not.

Regarding fair division (see, e.g., Brams \& Taylor, 1996; Bouveret, Chevaleyre, \& Maudet, 2016; Lang \& Rothe, 2015), while there is some work on chore division (see, e.g., Aziz, Rauchecker, Schryen, \& Walsh, 2017; Bogomolnaia, Moulin, Sandomirskiy, \& Yanovskaya, 2016, for recent work), not much is known about settings where an item can be seen as negative for an agent and positive for another one while a third agent does not care about receiving it; ${ }^{6}$ still, there are many pratical contexts where this assumption is plausible, such as the allocation of papers to reviewers. If there is no constraint on the allocation, then obviously an item will be assigned to an agent who likes it, provided there is at least one such agent; but if there are constraints (such as balancedness), then it may be the case that an agent gets an item she does not want even though someone expressed a positive preference for it.

We have then looked at several stability concepts in hedonic games with such preferences. The issue of incomparabilities that may remain is tackled by letting these incomparabilities be unresolved and introducing, inspired by the work on necessary and possible winners in voting (Konczak

\footnotetext{
${ }^{6}$ Recently, Aziz, Caragiannis, Igarashi, and Walsh (2019) studied, from a computational perspective, fair allocation of indivisible goods and chores where an agent may have either a negative or a positive utility for each item. In economics, Bogomolnaia, Moulin, Sandomirskiy, and Yanovskaya (2017) studied fair division of divisible items that they call "mixed manna" (containing both goods liked by everyone and bads disliked by everyone, but also items that are goods to some agents, yet bads or satiated to other agents).
} 
\& Lang, 2005; Xia \& Conitzer, 2011) ${ }^{7}$ the notions of necessity and possibility for known stability concepts. $^{8}$ We have analyzed the computational complexity of the existence and the verification problem of well-known stability concepts for the induced hedonic games. So far, with the help of these solution concepts we can verify whether a coalition structure is a "good" solution, compare two coalition structures, and decide whether there even exists such a coalition structure-sometimes only at great cost in terms of computational complexity, though.

For future work, we propose to consider other solution concepts and to solve the remaining open problems, especially regarding those entries in Table 3 where matching upper and lower bounds on the complexity of problems have not been found yet. One approach to tackle these open problems might be to apply the metaresults from the intriguing work of Peters and Elkind (2015) who establish relations between certain properties of preferences in hedonic games and NP-hardness of certain stability existence problems. Indeed, for our FEN-hedonic games we are able to show, as an easy consequence of the metaresults due to Peters and Elkind (2015), that NASH-STABILITYExistence, Individual-Stability-Existence, CoRE-STABility-Existence, and STRICTCORE-STABIlity-Existence are NP-hard (see the PhD thesis of Rey, 2016, for details). However, this does not say anything about the complexity of the corresponding variants with possible or necessary stability; the open problems in Table 3 remain.

Besides further pursuing the analysis initiated here, for future work we also propose to introduce the notion of partition correspondences with the purpose to actually identify "good" coalition structures as an output. In contrast to the original idea of hedonic games where coalitions form in a decentralized manner, a central authority might be used here, in order to decide which coalitions will "best" work together. This might, for example, be the case in a setting where the head of a department has to divide a group of employees into teams. The teams should be stable and/or should have high social welfare (in the sense that the team members are as happy as possible with their group to create a good working atmosphere).

Also, the various notions of altruism in hedonic games may be useful here, such as those introduced by Nguyen et al. (2016) where players care not only about their own preferences but also about their friends' preferences. While they focus on friend-oriented preferences to define altruistic hedonic games of various types, other (compact) representations of hedonic games might be used as well for this purpose, and we propose as a challenging task for future research to study altruism in FEN-hedonic games where the players' preferences on coalitions are based on the polarized responsive extension principle.

\section{Acknowledgments}

This work was supported in part by DFG grants RO-1202/\{14-1, 14-2, 15-1\}, by Agence Nationale de la Recherche under the "programme d'Investissements d'avenir" ANR-19-P3IA-0001 (PRAIRIE), by a DAAD-PPP/PHC PROCOPE project, and by COST Action IC1205 on Computational Social Choice.

\footnotetext{
${ }^{7}$ Other areas where the concepts of necessary and possible winners from voting has been applied are fair division (Aziz, Walsh, \& Xia, 2015; Baumeister, Bouveret, Lang, Nguyen, Nguyen, Rothe, \& Saffidine, 2017) and strategyproofness in judgment aggregation (Baumeister, Erdélyi, Erdélyi, \& Rothe, 2015).

${ }^{8} \mathrm{~A}$ different approach has been taken by Rothe et al. (2018) who consider comparability functions based on Bordalike scoring vectors in order to resolve these incomparabilities (see also Lang et al., 2015).
} 


\section{References}

Aziz, H., Brandl, F., Brandt, F., Harrenstein, P., Olsen, M., \& Peters, D. (2019). Fractional hedonic games. ACM Transactions on Economics and Computation, 7(2), Article 6, 6:1-6:29.

Aziz, H., Brandt, F., \& Harrenstein, P. (2013a). Pareto optimality in coalition formation. Games and Economic Behavior, 82, 562-581.

Aziz, H., Brandt, F., \& Seedig, H. (2013b). Computing desirable partitions in additively separable hedonic games. Artificial Intelligence, 195, 316-334.

Aziz, H., Caragiannis, I., Igarashi, A., \& Walsh, T. (2019). Fair allocation of indivisible goods and chores. In Proceedings of the 28th International Joint Conference on Artificial Intelligence, pp. 53-59. ijcai.org.

Aziz, H., Gaspers, S., Mackenzie, S., \& Walsh, T. (2015). Fair assignment of indivisible objects under ordinal preferences. Artificial Intelligence, 227, 71-92.

Aziz, H., Harrenstein, P., Lang, J., \& Wooldridge, M. (2016). Boolean hedonic games. In Proceedings of the 15th International Conference on Principles of Knowledge Representation and Reasoning, pp. 166-175. AAAI Press.

Aziz, H., Rauchecker, G., Schryen, G., \& Walsh, T. (2017). Algorithms for max-min share fair allocation of indivisible chores. In Proceedings of the 31st AAAI Conference on Artificial Intelligence, pp. 335-341. AAAI Press.

Aziz, H., \& Savani, R. (2016). Hedonic games. In Brandt, F., Conitzer, V., Endriss, U., Lang, J., \& Procaccia, A. (Eds.), Handbook of Computational Social Choice, chap. 15, pp. 356-376. Cambridge University Press.

Aziz, H., Walsh, T., \& Xia, L. (2015). Possible and necessary allocations via sequential mechanisms. In Proceedings of the 24th International Joint Conference on Artificial Intelligence, pp. 468474. AAAI Press/IJCAI.

Ballester, C. (2004). NP-completeness in hedonic games. Games and Economic Behavior, 49(1), $1-30$.

Banerjee, S., Konishi, H., \& Sönmez, T. (2001). Core in a simple coalition formation game. Social Choice and Welfare, 18, 135-153.

Baumeister, D., Bouveret, S., Lang, J., Nguyen, N., Nguyen, T., Rothe, J., \& Saffidine, A. (2017). Positional scoring-based allocation of indivisible goods. Journal of Autonomous Agents and Multi-Agent Systems, 31(3), 628-655.

Baumeister, D., Erdélyi, G., Erdélyi, O., \& Rothe, J. (2015). Complexity of manipulation and bribery in judgment aggregation for uniform premise-based quota rules. Mathematical Social Sciences, 76, 19-30.

Bilò, V., Fanelli, A., Flammini, M., Monaco, G., \& Moscardelli, L. (2014). Nash stability in fractional hedonic games. In Proceedings of the 10th International Workshop on Internet \& Network Economics, pp. 486-491. Springer-Verlag Lecture Notes in Computer Science \#8877.

Bilò, V., Fanelli, A., Flammini, M., Monaco, G., \& Moscardelli, L. (2015). On the price of stability of fractional hedonic games. In Proceedings of the 14th International Conference on Autonomous Agents and Multiagent Systems, pp. 1239-1247. IFAAMAS. 
Bogomolnaia, A., \& Jackson, M. (2002). The stability of hedonic coalition structures. Games and Economic Behavior, 38(2), 201-230.

Bogomolnaia, A., Moulin, H., Sandomirskiy, F., \& Yanovskaya, E. (2016). Dividing goods and bads under additive utilities. Tech. rep. arXiv:1608.01540v1 [cs.GT], ACM Computing Research Repository (CoRR).

Bogomolnaia, A., Moulin, H., Sandomirskiy, F., \& Yanovskaya, E. (2017). Competitive division of a mixed manna. Econometrica, 85(6), 1847-1871.

Bossong, U., \& Schweigert, D. (2006). Minimal paths on ordered graphs. Mathematica Slovaca, $56(1), 23-31$.

Bouveret, S., Chevaleyre, Y., \& Maudet, N. (2016). Fair allocation of indivisible goods. In Brandt, F., Conitzer, V., Endriss, U., Lang, J., \& Procaccia, A. (Eds.), Handbook of Computational Social Choice, chap. 12, pp. 284-310. Cambridge University Press.

Bouveret, S., Endriss, U., \& Lang, J. (2010). Fair division under ordinal preferences: Computing envy-free allocations of indivisible goods. In Proceedings of the 19th European Conference on Artificial Intelligence, pp. 387-392. IOS Press.

Brams, S., \& Sanver, R. (2009). Voting systems that combine approval and preference. In Brams, S., Gehrlein, W., \& Roberts, F. (Eds.), The Mathematics of Preference, Choice, and Order: Essays in Honor of Peter C. Fishburn, pp. 215-237. Springer.

Brams, S., \& Taylor, A. (1996). Fair Division: From Cake-Cutting to Dispute Resolution. Cambridge University Press.

Cechlárová, K., \& Hajduková, J. (2003). Computational complexity of stable partitions with Bpreferences. International Journal of Game Theory, 31(3), 353-364.

Cechlárová, K., \& Hajduková, J. (2004). Stable partitions with $\mathscr{W}$-preferences. Discrete Applied Mathematics, 138(3), 333-347.

Cechlárová, K., \& Romero-Medina, A. (2001). Stability in coalition formation games. International Journal of Game Theory, 29(4), 487-494.

Darmann, A., Elkind, E., Kurz, S., Lang, J., Schauer, J., \& Woeginger, G. (2018). Group activity selection problem with approval preferences. International Journal of Game Theory, 47(3), 767-796.

Delort, C., Spanjaard, O., \& Weng, P. (2011). Committee selection with a weight constraint based on a pairwise dominance relation. In Proceedings of the 2 nd International Conference on Algorithmic Decision Theory, pp. 28-41. Springer-Verlag Lecture Notes in Artificial Intelligence \#6992.

Dimitrov, D., Borm, P., Hendrickx, R., \& Sung, S. (2006). Simple priorities and core stability in hedonic games. Social Choice and Welfare, 26(2), 421-433.

Elkind, E., \& Rothe, J. (2015). Cooperative game theory. In Rothe, J. (Ed.), Economics and Computation. An Introduction to Algorithmic Game Theory, Computational Social Choice, and Fair Division, chap. 3, pp. 135-196. Springer-Verlag.

Elkind, E., \& Wooldridge, M. (2009). Hedonic coalition nets. In Proceedings of the 8th International Joint Conference on Autonomous Agents and Multiagent Systems, pp. 417-424. IFAAMAS. 
Faliszewski, P., Skowron, P., Slinko, A., \& Talmon, N. (2017). Multiwinner voting: A new challenge for social choice theory. In Endriss, U. (Ed.), Trends in Computational Social Choice, chap. 2, pp. 27-47. AI Access Foundation.

Garey, M., \& Johnson, D. (1979). Computers and Intractibility: A Guide to the Theory of NPCompleteness. W. H. Freeman and Company.

Kerkmann, A., \& Rothe, J. (2019). Stability in FEN-hedonic games for single-player deviations. In Proceedings of the 18th International Conference on Autonomous Agents and Multiagent Systems. IFAAMAS. To appear.

Konczak, K., \& Lang, J. (2005). Voting procedures with incomplete preferences. In Proceedings of the Multidisciplinary IJCAI-05 Workshop on Advances in Preference Handling, pp. 124-129.

Lang, J., Rey, A., Rothe, J., Schadrack, H., \& Schend, L. (2015). Representing and solving hedonic games with ordinal preferences and thresholds. In Proceedings of the 14th International Conference on Autonomous Agents and Multiagent Systems, pp. 1229-1237. IFAAMAS.

Lang, J., \& Rothe, J. (2015). Fair division of indivisible goods. In Rothe, J. (Ed.), Economics and Computation. An Introduction to Algorithmic Game Theory, Computational Social Choice, and Fair Division, chap. 8, pp. 493-550. Springer-Verlag.

Meyer, A., \& Stockmeyer, L. (1972). The equivalence problem for regular expressions with squaring requires exponential space. In Proceedings of the 13th IEEE Symposium on Switching and Automata Theory, pp. 125-129. IEEE Computer Society Press.

Nguyen, N., Rey, A., Rey, L., Rothe, J., \& Schend, L. (2016). Altruistic hedonic games. In Proceedings of the 15th International Conference on Autonomous Agents and Multiagent Systems, pp. 251-259. IFAAMAS. Also presented at the 7th International Workshop on Cooperative Games in Multiagent Systems (CoopMAS 2016), co-located with AAMAS 2016, and at the 6th International Workshop on Computational Social Choice (COMSOC 2016), Toulouse, France, June 2016, both with nonarchival proceedings.

Papadimitriou, C. (1995). Computational Complexity (Second edition). Addison-Wesley.

Peters, D. (2016). Complexity of hedonic games with dichotomous preferences. In Proceedings of the 30th AAAI Conference on Artificial Intelligence, pp. 579-585. AAAI Press.

Peters, D., \& Elkind, E. (2015). Simple causes of complexity in hedonic games. In Proceedings of the 24th International Joint Conference on Artificial Intelligence, pp. 617-623. AAAI Press/IJCAI.

Rey, A. (2016). Beyond Intractability: A Computational Complexity Analysis of Various Types of Influence and Stability in Cooperative Games. Ph.D. thesis, Heinrich-Heine-Universität Düsseldorf, Düsseldorf, Germany.

Rey, A., Rothe, J., Schadrack, H., \& Schend, L. (2016). Toward the complexity of the existence of wonderfully stable partitions and strictly core stable coalition structures in enemy-oriented hedonic games. Annals of Mathematics and Artificial Intelligence, 77(3), 317-333.

Roth, A. (1985). Common and conflicting interests in two-sided matching markets. European Economic Review, 27(1), 75-96.

Roth, A., \& Sotomayor, M. (1992). Two-sided Matching: A Study in Game-Theoretic Modeling and Analysis. Cambridge University Press. 
Rothe, J. (2005). Complexity Theory and Cryptology. An Introduction to Cryptocomplexity. EATCS Texts in Theoretical Computer Science. Springer-Verlag.

Rothe, J., Schadrack, H., \& Schend, L. (2018). Borda-induced hedonic games with friends, enemies, and neutral players. Mathematical Social Sciences, 96, 21-36.

Sen, A. (1970). Interpersonal aggregation and partial comparability. Econometrica, 38, 393-409.

Stockmeyer, L. (1976). The polynomial-time hierarchy. Theoretical Computer Science, 3(1), 1-22.

Sung, S., \& Dimitrov, D. (2007). On core membership testing for hedonic coalition formation games. Operations Research Letters, 35(2), 155-158.

Sung, S., \& Dimitrov, D. (2010). Computational complexity in additive hedonic games. European Journal of Operational Research, 203(3), 635-639.

Woeginger, G. (2013a). Core stability in hedonic coalition formation. In Proceedings of the 39th Conference on Current Trends in Theory and Practice of Computer Science, pp. 3350. Springer-Verlag Lecture Notes in Computer Science \#7741.

Woeginger, G. (2013b). A hardness result for core stability in additive hedonic games. Mathematical Social Sciences, 65(2), 101-104.

Xia, L., \& Conitzer, V. (2011). Determining possible and necessary winners given partial orders. Journal of Artificial Intelligence Research, 41, 25-67. 